\title{
Cramér-Rao Lower Bound Derivation and Performance Analysis for Space-Based SAR SMTI
}

\author{
by \\ Mamoon Rashid
}

A thesis submitted to the Faculty of Graduate and Postdoctoral

Affairs in partial fulfillment of the requirements

for the degree of

Master of Applied Sciences

M.A.Sc. Electrical and Computer Engineering

Department of Systems and Computer Engineering

Carleton University

Ottawa, Ontario, Canada, K1S 5B6

2015

(C) Mamoon Rashid, Ottawa, Canada, 2015 


\begin{abstract}
This thesis develops the Cramér-Rao Lower Bound (CRLB) for multi-channel spaceborne synthetic aperture radar (SAR) system and provides surface moving target indication (SMTI) performance analysis. CRLB provides a lower bound on the achievable variance of any unbiased estimator. An estimator that achieves this bound is called efficient, however, there is no guarantee that an efficient estimator can be found. Nonetheless, the theoretical variance of the efficient estimator provides a good estimate of the capability of the system and serves as a valuable system performance validation tool. Even if an efficient estimator cannot be found, for radar systems the CRLB provides a necessary, but not sufficient design baseline for measurement parameters such as the number of sub-apertures for transmit and receive, power levels, pulse-repetition frequency (PRF), etc.

A multi-channel moving target signal model is derived in satellite earth-fixed earthcentered coordinate system. This model is used in space-time adaptive processing (STAP) approaches for SMTI. A statistical model of the received signal is formed using the derived deterministic target signal, and Gaussian distributions for noise and clutter. CRLB for the statistical model and target parameters is derived by solving the derivatives. The non-trivial derivatives are also verified using a numerical method. CRLB is then used to analyse the SMTI performance of RADARSAT-2, RADARSAT constellation mission (RCM) satellite, and a proposed satellite called "TestSAT", under a variety of switching/toggling modes. The results confirm the SMTI capability of RADARSAT-2 demonstrated previously [1,2], and the optimal switching/toggling mode [3 5]. The simulations for RCM demonstrate that its SMTI capability will be far inferior to RADARSAT-2. However, by slightly changing the parameters of RCM, as was done for TestSAT, it was shown that an SMTI performance that is comparable to that of RADARSAT-2 can be theoretically achieved with a smaller aperture size and lower transmitted power.

The main contributions of this thesis include the derivation of the CRLB for multichannel space-borne SAR, and theoretical SMTI performance analysis using CRLB. The goal of the analysis was two-fold: i) to find the SMTI performance limits of realistic systems over different switching/toggling configurations, and ii) to use CRLB as a benchmark tool to determine if it is possible to have a system that consumes less power than an existing system and provides a comparable or better SMTI performance. The theoretical results demonstrate the usefulness of CRLB as a tool in the theoretical performance evaluation of different systems and switching/toggling schemes for SMTI.
\end{abstract}




\section{Acknowledgements}

First and foremost, I would like to thank my parents for their love and support throughout my life. Thank you both for giving me the strength to pursue my intellectual interests. Special thanks to my elder brother for teaching me how to struggle and persevere against overwhelming odds.

I would like to thank Radar Systems section at Defence Research and Development Canada - Ottawa for the opportunity and assistance on this thesis. In particular, I would like to thank Dr. Ishuwa Sikaneta for countless discussions, mentoring, and support in completing the thesis. Thanks to Dr. Christoph Gierull and Dr. Chuck Livingstone for their explanations and support. Thanks to Dr. Shen Chiu for providing me with various texts and resources. Last, but not least, I would like to thank my supervisor Dr. Richard Dansereau for guidance and support. 


\section{Contents}

$\begin{array}{lll}1 & \text { Introduction } & 5\end{array}$

1.1 Research Objectives . . . . . . . . . . . . . . . . . . . . . . . 6

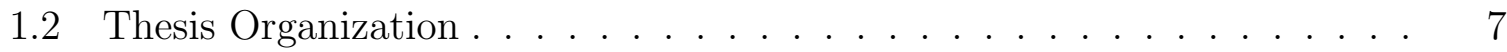

1.3 Thesis Contributions . . . . . . . . . . . . . . . . . . . . . . . . . 8

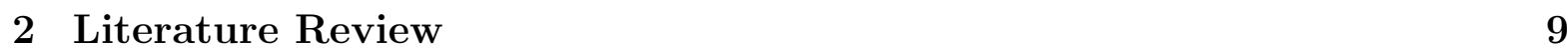

2.1 SAR Theory . . . . . . . . . . . . . . . . . . . . . . . . . . . . . . . . 9 9

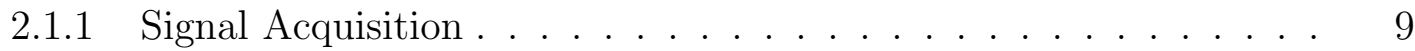

2.1 .2 Signal in the Range Direction . . . . . . . . . . . . . . . . . . 11

2.1 .3 Signal in the Azimuth Direction . . . . . . . . . . . . . . . . . . . 13

2.1 .4 SAR Processing . . . . . . . . . . . . . . . . . . . . . . . . 14

2.2 Moving Target Signal Model . . . . . . . . . . . . . . . . . . . . . . . . . 16

2.2 .1 Range Equation . . . . . . . . . . . . . . . . . . . . . 16

2.2 .2 Antenna Array Pattern . . . . . . . . . . . . . . . . . . . 21

2.2 .3 Signal in Doppler Domain . . . . . . . . . . . . . . . . . . . . 23

2.2 .4 Azimuth Parameters . . . . . . . . . . . . . . . . . . 27

2.3 Methods for Surface Moving Target Indication . . . . . . . . . . . . . . . 29

2.3.1 Multi-channel SAR surface moving target indication (SMTI) . . . 29

2.3 .2 Along-Track Interferometry . . . . . . . . . . . . . . . . 31

$2.3 .3 \quad$ Extended Displaced Phase Center Antenna . . . . . . . . . . . . . . 31

2.3 .4 Imaging Space-Time Adaptive Processing . . . . . . . . . . . . . . 34

3 Cramér-Rao Lower Bound Derivation 38

3.1 Statistical model of clutter and noise . . . . . . . . . . . . . . . . . . . . 39

3.2 Partial Derivatives . . . . . . . . . . . . . . . . . . . . . . . . . . . . 40

3.2.1 Partial derivative of $\mathbf{s}(\Theta)$ with respect to $\sigma_{s}$. . . . . . . . . . . 40

$3.2 .2 \quad$ Partial derivative of $\mathbf{s}(\Theta)$ with respect to $\Delta_{s} \ldots \ldots$. . . . . . . 41 


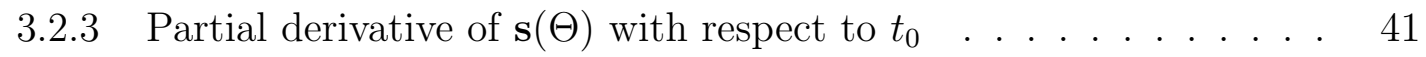

$3.2 .4 \quad$ Partial derivative of $\mathbf{s}(\Theta)$ with respect to $V_{\mathrm{x}} \ldots \ldots$. . . . . . . . 42

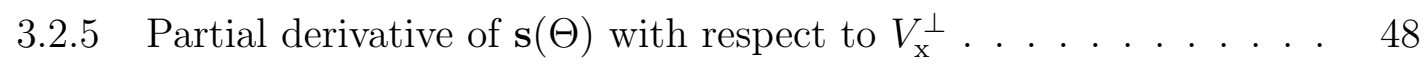

\begin{tabular}{|lrl}
4 & SMTI Performance Analysis & 53
\end{tabular}

4.1 System Parameters . . . . . . . . . . . . . . . . . . . . . . . 54

4.2 SMTI Performance Analysis . . . . . . . . . . . . . . . . . . 57

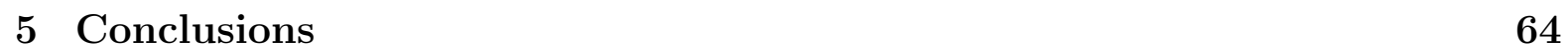

5.1 Thesis Contributions . . . . . . . . . . . . . . . . . . . . 64

5.2 Suggestions for Future Work . . . . . . . . . . . . . . . . . . . . . . 65

\begin{tabular}{|ll}
\hline A Space-based Geometry & $\mathbf{7 2}$
\end{tabular}

A.1 Coordinate Systems . . . . . . . . . . . . . . . . . . . 72

A.2 Coordinate Transformations . . . . . . . . . . . . . . . . . . . . 74

A.2.1 Transformation from I to E . . . . . . . . . . . . . . . . . . . . 74

A.2.2 Transformation from I to S . . . . . . . . . . . . . . . . . . 76

A.2.3 Transformation from $\mathrm{S}$ to $\mathrm{A}$. . . . . . . . . . . . . 76

A.2.4 Transformation from A to E . . . . . . . . . . . . . . 76

A.3 Satellite Position and Velocity Vectors . . . . . . . . . . . . . . . . 77

A.4 Moving Target . . . . . . . . . . . . . . . . . . . 80

A.4.1 Antenna Look Direction . . . . . . . . . . . . . . . . . . . . . . 82

B Azimuth Parameters from Antenna Theory 85

B.1 Real Aperture . . . . . . . . . . . . . . . . . . . . . . . 85

B.2 Synthetic Aperture . . . . . . . . . . . . . . . . . . . 87

\begin{tabular}{|lr|}
\hline C Stationary Phase Approximation & 89
\end{tabular}

\begin{tabular}{ll}
\hline D MODEX Modes & 91
\end{tabular}

D.1 Space-Time Sampling . . . . . . . . . . . . . . . . . . . . . . . . . . . 93

\begin{tabular}{lll}
\hline E & SNR and CNR & 95
\end{tabular}

E.1 Signal-To-Noise Ratio . . . . . . . . . . . . . . . . . . . . . . . . . . 95

E.1.1 Integrated SNR . . . . . . . . . . . . . . . . . . 97

E.2 Clutter-To-Noise Ratio . . . . . . . . . . . . . . . . . . . . . 98 


\section{List of Tables}

4.1 RADARSAT-2 Parameters . . . . . . . . . . . . . . . . . . 54

4.2 RADARSAT-2 Switching Parameters . . . . . . . . . . . . . . . . . 54

4.3 RCM Parameters . . . . . . . . . . . . . . . . . . . . . 55

$4.4 \quad$ RCM Switching Parameters $\ldots \ldots \ldots \ldots$

4.5 TestSAT Parameters $\ldots \ldots \ldots \ldots \ldots \ldots$

4.6 TestSAT Switching Parameters $\ldots \ldots \ldots \ldots$. . . . . . . . . . 56 


\section{List of Figures}

$2.1 \quad$ Acquisition Geometry.$\ldots \ldots \ldots$

$2.2 \quad$ Azimuth Signal $\ldots \ldots \ldots \ldots$

2.3 Imaging Geometry $\ldots \ldots \ldots \ldots \ldots \ldots \ldots \ldots$

$2.4 \quad$ ISTAP Clutter Filter . . . . . . . . . . . . . . . . . . . . . . . . 37

$3.1 \quad$ Partial derivative with respect to parameter $V_{\mathrm{x}}$ at $1 \mathrm{~Hz} . \ldots \ldots \ldots$

$3.2 \quad$ Partial derivative with respect to parameter $V_{\mathrm{x}}$ at $2 \mathrm{~Hz} . \ldots \ldots \ldots .46$

3.3 Partial derivative with respect to parameter $V_{\mathrm{x}}$ at $3 \mathrm{~Hz} . \ldots . . . .44$

3.4 Error term for the numerical and theoretical derivative of $V_{\mathrm{x}} . \ldots \ldots 47$

$3.5 \quad$ Partial derivative with respect to parameter $V_{\mathrm{x}}^{\perp}$ at $1 \mathrm{~Hz} . \quad \ldots \ldots . . .51$

$3.6 \quad$ Partial derivative with respect to parameter $V_{\mathrm{x}}^{\perp}$ at $2 \mathrm{~Hz}$. . . . . . . . . . 51

$3.7 \quad$ Partial derivative with respect to parameter $V_{\mathrm{x}}^{\perp}$ at $3 \mathrm{~Hz} . \quad \ldots \ldots . . \quad 52$

3.8 Error term for the numerical and theoretical derivative of $V_{\mathrm{x}}^{\perp} . \ldots \ldots 2$

4.1 RADARSAT-2 ISTAP clutter filters for MODEX modes. . . . . . . . . . 58

4.2 RADARSAT-2 CRLB for across-track velocity estimation. . . . . . . . . 59

4.3 RCM ISTAP clutter filters for MODEX modes. . . . . . . . . . . . . 60

$4.4 \quad$ RCM CRLB for across-track velocity estimation. . . . . . . . . . . . 61

4.5 TestSAT ISTAP clutter filters for MODEX modes. . . . . . . . . . . . 62

4.6 TestSAT CRLB for across-track velocity estimation. . . . . . . . . . 63

A.1 Coordinate Systems . . . . . . . . . . . . . . . . . . . . . . . . 75

A.2 Moving Target Model . . . . . . . . . . . . . . . . . . . . . . . . 81

A.3 Rotation between $\mathrm{A}$ and $\mathrm{D} \ldots \ldots \ldots$. . . . . . . . . . . . 83

B.1 Linear array antenna radiation measurement . . . . . . . . . . . . . 85

B.2 Synthetic Aperture . . . . . . . . . . . . . . . . . . . . 88

D.1 radarsat2-modex-modes . . . . . . . . . . . . . . . . . . . . . . 92 
D.2 modex-modes-sampling . . . . . . . . . . . . . . . . . . . . . . . 94 


\section{Nomenclature}

$t_{a} \quad$ Refers to azimuth time (slow-time) in the two-dimensional expression of the signal.

$\dagger \quad$ Hermitian transpose operator.

$r_{e} \quad$ Radius of Earth.

$u_{c} \quad$ Look direction to stationary clutter target.

$u_{t} \quad$ Look direction to the moving target.

$N_{f} \quad$ Noise figure.

$\omega_{e} \quad$ Angular rotation rate of Earth.

$\omega_{s} \quad$ Angular rotation rate of the satellite.

$R_{0} \quad$ The point target range when the target in the center of the beam (i.e. at zero Doppler time).

$L_{s} \quad$ Power losses.

$t_{r} \quad$ Refers to range time (fast-time) in the two-dimensional expression of the signal.

$G_{r x} \quad$ Receiver gain.

$h_{s} \quad$ Distance from the surface of the Earth to the radar platform.

$\theta \quad$ Incidence angle between range vector and the normal to the earth's surface.

$\theta_{i} \quad$ Inclination angle of the satellite orbit.

$\theta_{s q} \quad$ Beam squint angle.

$G_{t x} \quad$ Transmit gain. 
$P_{t x} \quad$ Transmit power.

$V_{\mathrm{a}} \quad$ The satellite velocity in satellite ECEF coordinate system.

$\vec{d}(u)$ The direction-of-arrival (DOA) vector.

$V_{\text {eff }} \quad$ The effective radar velocity, which is used to model the curved-earth geometry for an accurate representation of the range equation in space-borne applications.

$V_{\mathrm{g}} \quad$ The velocity of the radar beam as it moves along the ground.

$V_{\text {rel }} \quad$ The relative velocity between the moving target and the satellite.

$V_{\mathrm{rt}}^{\perp} \quad$ The radial velocity component of the moving target. The direction of velocity is along the line-of-sight (los) vector.

$V_{\mathrm{x}} \quad$ The along-track velocity component of the moving target, which is in the direction parallel to the satellite velocity vector.

$V_{\mathrm{x}}^{\perp} \quad$ The across-track velocity component of the moving target, which is in the crossrange direction.

$V_{\mathrm{y}} \quad$ The component of across-track velocity of the moving target that is in the direction along the positive $\mathrm{y}$-axis in satellite ECEF coordinate system (D).

$V_{\mathrm{z}} \quad$ The component of along-track velocity of the moving target that is in the direction along the positive z-axis in satellite ECEF coordinate system (D).

$A_{i}(u)$ The look-direction dependent antenna pattern for $i^{t} h$ channel.

$r_{s} \quad$ Distance from the center of the earth to the center of the platform.

$r_{n} \quad$ Distance from the center of the platform to the $n^{\text {th }}$ antenna phase center.

$t \quad$ Refers to slow-time in the one-dimensional representation of the signal.

$y_{0} \quad$ The y-component of the moving target position vector at broadside time in satellite ECEF coordinates (D)*. (check time)

$z_{0} \quad$ The z-component of the moving target position vector at broadside time in satellite ECEF coordinates (D)*. (check time) 


\section{Acronyms}

ATI along-track interferometry

CFAR constant false alarm rate

CPI coherent processing interval

CRLB Cramér-Rao Lower Bound

CSA chirp scaling algorithm

DPCA displaced phase center antenna

ECEF earth-centered earth-fixed coordinate system

EDPCA extended displaced phase center antenna

FM frequency modulated

GLRT generalized likelihood ratio test

GMTI ground moving target indication

ISTAP imaging space time adaptive processsing

MTI moving target indication

PRF pulse repetition frequency

PRI pulse repetition interval

RCM RADARSAT constellation mission

RCMC range cell migration correction 
RCS radar cross section

RDA range doppler algorithm

SAR synthetic aperture radar

SBR space-borne radar

SMTI surface moving target indication

SNR Signal to noise ratio

STAP space time adaptive processing 


\section{Chapter 1}

\section{Introduction}

Synthetic-aperture radar (SAR) is a form of radar that is typically used to produce two-dimensional images for environmental monitoring, earth-resource mapping, military systems, and a variety of other applications [6,7]. The discovery of SAR is generally credited to Carl Wiley of Goodyear Aerospace, who suggested the use of Doppler shifts to obtain a fine resolution in the cross-range direction. SAR was first developed for military surveillance and reconnaissance in the 1950s, however it wasn't until the 1970s that the technology was released to the civilian community. Since then, many airborne and satellite SAR systems have been deployed for a variety of applications in military and remote sensing [7]. The main advantages of $\mathrm{SAR}$ over optical sensors are as follows.

- Unlike most optical sensors, a SAR sensor carries its own coherent illumination source, and therefore works equally well in darkness.

- SAR sensors can emit electro-magnetic (EM) waves with frequencies that can pass through clouds and precipitation without much power loss.

- The emitted EM waves have different scattering characteristics, and therefore provide different information about the imaged surface than optical sensors.

- SAR sensors perform pulse-to-pulse coherent integration that provides target phase information, which is necessary for interferometry and phase-based moving target detection. Optical imaging sensors are typically incoherent and therefore don't provide any useful phase information.

There is ongoing research in moving target detection and estimation methods using

space-borne radar (SBR) SAR systems [1,2,4]. SBR systems are much different than the 
widely existing airborne SAR systems in terms of geometry and system architecture. As a result, these systems face different challenges than airborne systems. For example, the long distances involved between the sensor and the scene of interest (typically around $1000 \mathrm{~km}$ ) cause the SNR of the moving object to be very low. Furthermore, the number of physical receive channels on these systems are limited due to the cost. RADARSAT-2 and TerraSAR-X, which are two SBR systems capable of moving target detection and estimation, have only two physical receive channels. Two channels are insufficient to coherently cancel or suppress unambiguous clutter and estimate target parameters [4].

In SAR literature, there are two general classes of moving target detection and estimation methods for SBR systems. In the first class, the methods are generally derived from the concept of along-track interferometry (ATI) [8 and displaced phase center antenna (DPCA) [9]. The detection of moving target is performed on each range-azimuth pixel in the SAR processed images by considering the differences in either the amplitude, phase or both between different receive channels.

The second class of methods are derived from a well-known technique known as space-time adaptive processing (STAP) [10-12]. This method is typically used in radar systems that are used in environments that suffer from high interference such as nonstationary clutter and jamming. One efficient implementation of STAP is post-doppler STAP, which applies clutter cancellation and target detection in the Doppler domain for each independent range Doppler cell [11]. In [4], post-doppler STAP is extended for multichannel space-based SAR systems. The method derived is called imaging space time adaptive processsing (ISTAP) and it provides several theoretical advantages over the methods derived from ATI and DPCA.

\section{$1.1 \quad$ Research Objectives}

For an unbiased parameter estimator, CRLB provides a lower bound on the achievable variance. An estimate is said to be efficient if it achieves this bound. CRLB is a valuable tool to test and analyse the performance of a signal model. The variance of the efficient estimator gives the theoretical capability of the system. Even if an efficient estimator cannot be found, the CRLB provides a necessary but not sufficient design baseline for system parameters. Furthermore, CRLB can also be used to test the performance and the feasibility of the system. The feasibility of a system is defined as the ability of the system to perform SMTI. The CRLB can provide the feasibility of the system by providing the variance estimates of different target parameters. If the variance estimates 
are within some given criteria then the system is accepted as feasible, otherwise the system is rejected as unfeasible. For example, if the CRLB yields an across-track velocity standard deviation greater than the pre-determined criterion of $10 \mathrm{~m} / \mathrm{s}$, than the system can be rejected as unfeasible.

In this thesis, the CRLB associated with the signal model of a moving target in a multi-channel space-borne SAR system will be derived and analysed. The signal model makes two main assumptions

1. The SBR SAR system operates in the "standard" strip-map mode.

2. The SBR SAR is a pulse Doppler radar system.

The signal model used in this thesis is general in the sense that it does not assume a particular space-borne system (i.e. the parameters of the system are not fixed), and it does not assume any specific switching/toggling scheme for SMTI 1 .

In many target detection and estimation systems, some assumptions are made about the system performance over target parameters. For example, it is normally assumed that the estimate of the along-track velocity of the target will be very poor. Due to this assumption, some of the proposed algorithms don't even consider estimating this parameter. The CRLB can test such assumptions and provide a theoretical foundation for estimating the parameters.

\subsection{Thesis Organization}

A preliminary literature review is performed in Chapter 2, where the signal model for ISTAP is derived from earth and satellite geometry. Additionally, moving target detection and estimation methods are discussed in this chapter. A statistical model for ISTAP, and the CRLBs are derived in Chapter 3. The signal model does not assume a particular space-borne system or any specific switching/toggling scheme for SMTI. In Chapter 4, performance analysis using CRLB of RADARSAT-2, Radar Constellation Mission (RCM), and a hypothetical system called "TestSat" is performed. Thesis conclusions and suggestions for future work are listed in Chapter 5.

\footnotetext{
${ }^{1}$ System parameters include platform speed, orbit altitude, transmitted power, etc.
} 


\subsection{Thesis Contributions}

This thesis contributes to the area of space-borne SAR SMTI. Specifically, the CRLB for a statistical model of multi-channel moving target signal in the presence of clutter and noise is derived and verified. CRLB is then used to test the SMTI performance using the across-track velocity variance estimate, for three different systems under a variety of switching and toggling schemes.

To the best of my knowledge, the CRLB for the type of general signal model used in this thesis, which does not make any assumptions about the space-borne system parameters or specific switching/toggling strategies, has not been derived or presented in any previous work. Similar work includes target velocity variance estimates for specific switching/toggling schemes and systems. CRLB plots for target position variance for RADARSAT-2 and TerraSAR-X, under specific switching/toggling schemes are presented in [13]. These results are a special case of the results presented in this thesis. CRLB plots for the target radial speed, along-track speed, and position for RADARSAT2 under specific switching/toggling schemes are provided in [2]. The imaging geometry used in this work is a bit different than the one used in this thesis. Similar CRLB plots for RADARSAT-2 under specific switching/toggling schemes are also provided in [1, 14], which can also be seen as a special case of the results in this thesis. None of the results presented these works provide an epression for CRLB as provided in this thesis. These results are therefore limited and cannot be used to test the SMTI performance of any other system, under any other switching/toggling strategy, which is one of the main

goals of this thesis. The contributions of this thesis allow the analysis and evaluation of different SBR SAR systems and switching/toggling schemes as presented in Chapter 4. 


\section{Chapter 2}

\section{Literature Review}

This chapter presents the summary of literature on the topics relevant to the problem of surface moving target indication (SMTI) with space-based multi-channel SAR. It begins with a discussion of basic SAR theory in section 2.1. Methods for moving target detection using space-based radar are presented in section 2.3 .

\subsection{SAR Theory}

The purpose of this section is to present the principles of SAR. The section begins with an explanation of SAR signal acquisition in section 2.1.1. A discussion of the signal in the range direction is presented in section 2.1.2 and in the azimuth direction in section 2.1.3. Lastly, an outline of SAR processing for image formation is presented in section 2.1 .4 ,

Note that although there are many different types of radar systems, the discussion in this thesis only pertains to pulsed linear frequency modulated (FM) radar systems.

\subsubsection{Signal Acquisition}

A simplified geometry for SAR signal acquisition is shown in Figure 2.1 (7). A radar is mounted on a moving platform that moves along the azimuth or along-track direction with a constant speed1. Point "P1" in the figure represents the data acquisition start time. When the sensor reaches this point, it emits an electromagnetic (EM) pulse through

\footnotetext{
${ }^{1}$ The terms "radar" and "sensor" are used interchangeably. A "moving platform" is typically an aircraft or a satellite.
} 


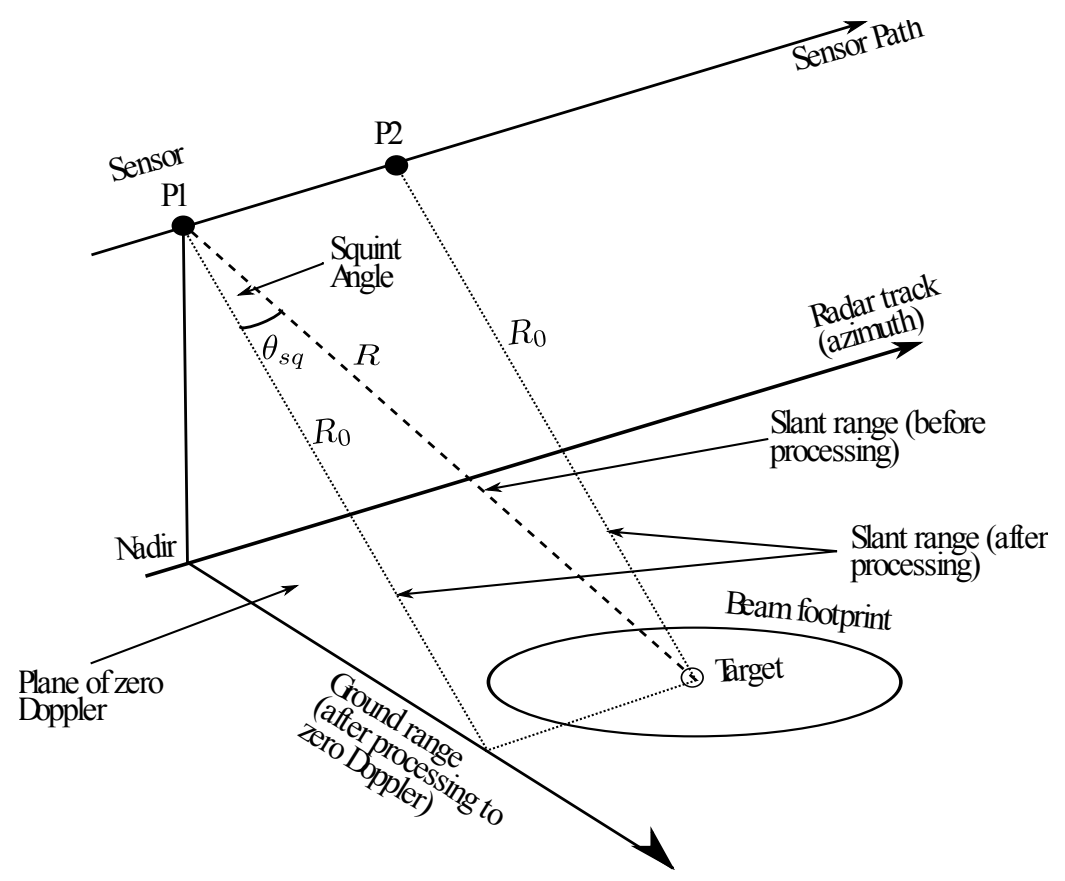

Figure 2.1: Signal acquisition geometry.

the antenna. The pulse hits the surface and is reflected (scattered). The reflected pulse travels back to the antenna, where it is converted into a voltage (i.e., continuous-time signal) and digitized. The received pulse has the same shape as the transmitted pulse, but is much weaker due to the losses, and has a frequency shift due to the relative speed of the sensor and the scatterer. Another pulse is transmitted when the sensor reaches point "P2" and then the reflected pulse is received by the antenna in a similar manner. In this way, continuous sections of the scene are imaged by repeatedly transmitting and receiving pulses at a constant time interval. This constant time interval between two transmitted or received pulses is known as pulse repetition interval (PRI) and the reciprocal of this interval is called the pulse repetition frequency (PRF). The coherent summation of the received pulses allows the construction of a "synthetic aperture" that is much longer than the physical antenna length. The received echo signal data forms a two-dimensional (2-D) data matrix of complex samples. The first dimension corresponds to the SAR signal in the range direction or fast-time. Each range line in the SAR data corresponds to a received echo after it has been amplified, down converted to baseband, and digitized. The collection of range lines, each received after every PRI, forms the second dimension of the data matrix, which is known as azimuth or slow-time. The received raw SAR data doesn't provide any useful information until SAR processing has 
been performed to obtain a focused image. SAR processing requires a highly accurate model of the transmitted signal and the imaging geometry.

\subsubsection{Signal in the Range Direction}

There are different types of radar systems, however the focus of this study is on pulse Doppler radars that send out a linear frequency modulated (FM) pulse [15]. Pulse Doppler radars are typically used in space-based SAR systems such as RADARSAT-2. These systems transmit a linear FM pulse in the range direction 7

$$
s_{\text {pul }}\left(t_{r}\right)=w_{r}\left(t_{r}\right) \cos \left(2 \pi f_{0} t_{r}+\pi K_{r} t_{r}^{2}\right),
$$

where $K_{r}$ is the FM rate of the pulse, $f_{0}$ is the starting frequency of the transmitted pulse, and $w_{r}$ is the pulse envelope, which is usually approximated by a rectangular function of the form

$$
w_{r}\left(t_{r}\right)=\operatorname{rect}\left(\frac{t_{r}}{T_{r}}\right)
$$

where $T_{r}$ is the pulse duration. Linear FM signals are typically used in radar applications in order to apply pulse compression, which is a type of spread spectrum method designed to minimize peak power requirements, maximize signal-to-noise ratio, and provides a fine resolution of the sensed object [7]. The bandwidth of the pulse, which is defined as the range of frequencies spanned by the significant energy of the chirp, is the product of the FM rate and the pulse duration

$$
B W=\left|K_{r}\right| T_{r}
$$

The complex sampling rate of the demodulated received pulse must be higher than the pulse bandwidth. The pulse resolution, which is defined as the spread between the two $-3 \mathrm{~dB}$ points of the pulse compressed signal, is given in meters by

$$
\rho_{r}=\frac{\mathrm{c}}{2} \frac{0.886 \gamma_{w, r}}{\left|K_{r}\right| T_{r}} \approx \frac{\mathrm{c}}{2} \frac{1}{\left|K_{r}\right| T_{r}},
$$

where "c" is the propagation speed of the pulse (speed of light), and $\gamma_{w, r}$ is the broadening factor of the tapered window that is applied when demodulating to baseband. It is important to note here that the range resolution is inversely proportional to the pulse bandwidth.

The received pulse, $s_{r}\left(t_{r}\right)$, is modelled as a convolution of the transmitted pulse and ground reflectivity. The ground reflectivity $g_{r}\left(t_{r}\right)$ can be modelled as a point target at 
a distance $R_{0}$ from the radar, and with a magnitude that is a function of the two-way antenna beam pattern and the target reflectivity.

$$
g_{r}\left(t_{r}\right)=S(\alpha) \delta\left(t_{r}-\frac{2 R_{0}}{\mathrm{c}}\right)
$$

where $S(\alpha)$ is the function that models the amplitude of ground target reflectivity, and $\alpha$ is the target's radar cross-section (RCS). The quantity $\frac{2 R_{0}}{\mathrm{c}}$ is the delay time of the signal for the point target. Using equations 2.1 and 2.5, the received signal from a point target can be written as

$$
\begin{aligned}
s_{r}\left(t_{r}\right) & =g_{r}\left(t_{r}\right) * s_{p u l}\left(t_{r}\right), \\
& =\int_{-\infty}^{\infty} S(\alpha) \delta\left(t_{r}-\frac{2 R_{0}}{\mathrm{c}}-\tau\right) w_{r}(\tau) \cos \left(2 \pi f_{0} \tau+\pi K_{r} \tau^{2}\right) d \tau \\
& =S(\alpha) w_{r}\left(t_{r}-\frac{2 R_{0}}{c}\right) \cos \left(2 \pi f_{0}\left(t_{r}-\frac{2 R_{0}}{\mathrm{c}}\right)+\pi K_{r}\left(t_{r}-\frac{2 R_{0}}{\mathrm{c}}\right)^{2}\right) .
\end{aligned}
$$

The received signal from a point target in Equation 2.6c is derived after applying the sifting property of the dirac-delta function in Equation 2.6b [7]. Note that the received signal in Equation 2.6c is the scaled and time-shifted version of the transmitted pulse in Equation 2.1.

The received pulse is demodulated and mixed to baseband upon arrival through a quadrature demodulation or a similar method. A detailed discussion of the quadrature demodulation process can be found in [7,16]. The process essentially removes the carrier frequency $\left(\cos \left(2 \pi f_{0} t_{r}\right)\right)$, and preserves the phase of the received target signal relative to the transmit signal. The received pulse from a single point target at baseband after demodulation can be written as

$$
s_{r}\left(t_{r}\right)=S^{\prime}(\alpha) w_{r}\left(t_{r}-\frac{2 R_{0}}{\mathrm{c}}\right) \exp \left(-j 4 \pi \frac{R_{0}}{\lambda}\right) \exp \left(j \pi K_{r}\left(t_{r}-\frac{2 R_{0}}{\mathrm{c}}\right)^{2}\right),
$$

where the function $S^{\prime}(\alpha)$ is a complex quantity given by $S^{\prime}(\alpha)=S(\alpha) \exp (j \psi)$ and $\psi$ is the phase change in the recieved signal that is introduced by the reflection from the surface [2, 16, 17]. This phase change is assumed to be constant for a given reflector within the radar illumination time. After demodulation, the received signal is sampled in range, where the complex sampling rate, $F_{r}$, should be greater than the signal bandwidth

$$
F_{r} \geq\left|K_{r}\right| T_{r}
$$

The received signal is typically sampled above the Nyquist criterion (oversampled) in order to adequately preserve the information in the continuous-time signal [7]. 


\subsubsection{Signal in the Azimuth Direction}

As the radar advances along its flight path in the azimuth direction, it transmits a pulse after each PRI, Figure 2.2 shows a radar and its antenna azimuth pattern with zerosquint angle as it moves along in the azimuth direction over a point target. As the radar approaches position "A", the target is just entering the main-lobe of the beam. The received signal strength, which is shown in the middle part of the figure, increases until the target lies in the center of the beam, at position "B". After this point, the signal strength decreases until the target lies in the first null of the beam pattern, at position "C". Due to the non-ideal antenna beam pattern, a small amount of energy from the point target will be received from side lobes of the antenna beam pattern. This energy from the side lobes, and as well as some energy from the outer edges of the main lobe, contributes to the azimuth ambiguities in the processed image [7, 16]. Due to the sensor trajectory in the azimuth direction, the range to the target in Equation 2.7, $R_{0}$, becomes a function of azimuth time, $t_{a}$. Therefore, the complex-valued received signal from a point target after taking into account the sensor trajectory can be written as

$$
\begin{array}{r}
s_{r}\left(t_{r}, t_{a}\right)=S^{\prime}\left(\alpha, t_{a}\right) w_{r}\left(t_{r}-\frac{2 R\left(t_{a}\right)}{\mathrm{c}}\right) w_{a}\left(t_{a}-t_{0}\right) \exp \left(-j 4 \pi \frac{R\left(t_{a}-t_{0}\right)}{\lambda}\right) \\
\exp \left(j \pi K_{r}\left(t_{r}-\frac{2 R\left(t_{a}\right)}{\mathrm{c}}\right)^{2}\right)
\end{array}
$$

where $w_{a}\left(t_{a}\right)$ is the two-way antenna beam pattern and $t_{0}$ is the "beam center crossing time" 17, 18. Note that both of these parameters depend on the antenna squint angle, $\theta_{s q}$, and the imaging geometry that is discussed in Section 2.2. Target amplitude $S^{\prime}\left(\alpha, t_{a}\right)$ changes with azimuth time due to the shape of the azimuth beam and how it passes over the target $[16,17]$. 


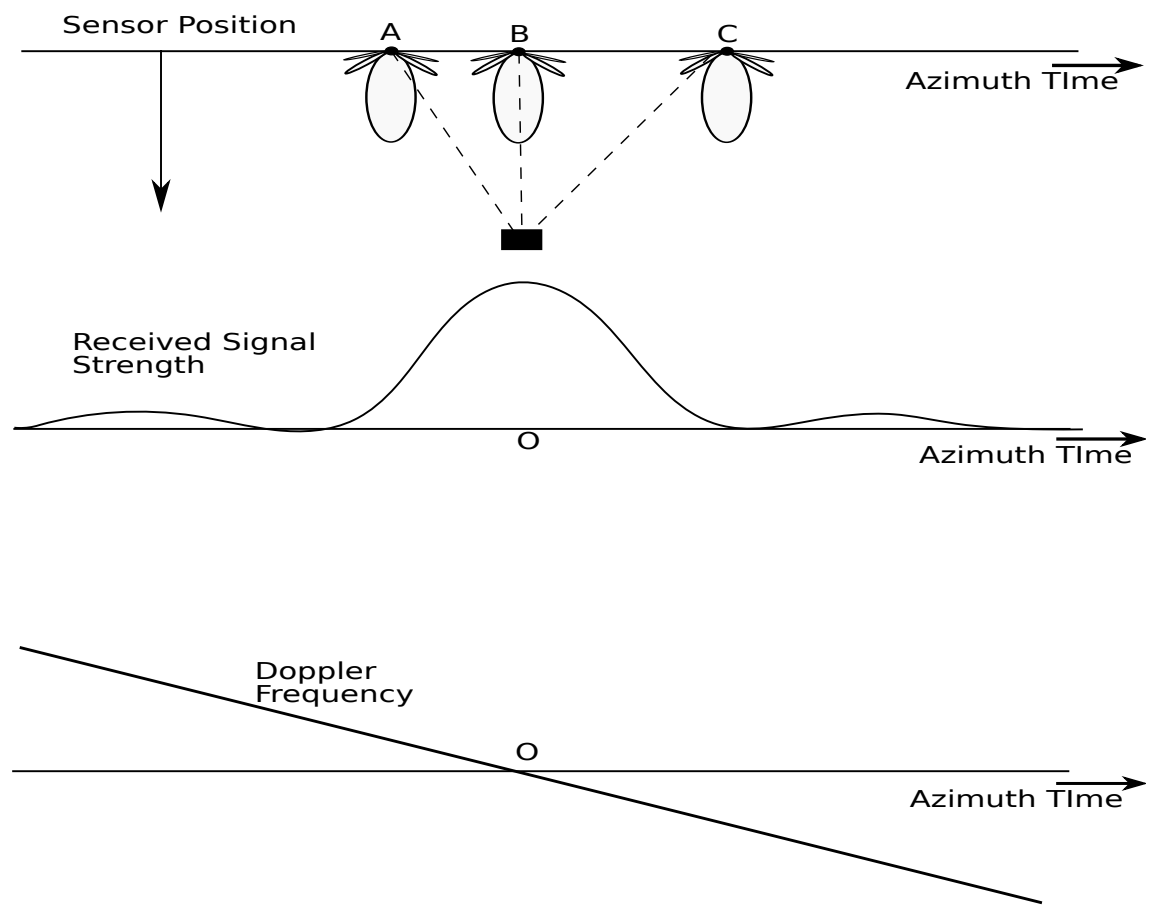

Figure 2.2: Radar trajectory in the azimuth direction and its effect on the signal strength and the Doppler frequency. [17]

The bottom plot in Figure 2.2 shows the SAR azimuth frequency history of the target. This azimuth frequency, which is also known as Doppler frequency after the wellknown Doppler effect, is a function of the target's radial velocity relative to the radar. A precise definition of the target's relative radial velocity is discussed in Section 2.2 . At this point, it is important to note that when the target is approaching the radar or when the distance from the target to the antenna is decreasing, the Doppler frequency is positive. Conversely, when the target is receding or when the distance from the target to the radar is increasing, the frequency is negative. This is shown in the negative slope of the Doppler frequency plot in Figure 2.2 16 18.

\subsubsection{SAR Processing}

For most applications, the raw SAR data doesn't provide any useful information. It is only after a focused SAR image has been obtained that the data can be useful. A brief summary of SAR image formation is discussed in this section. Many different algorithms have been developed that are used for SAR image formation. Some of the widely used algorithms include the Range-Doppler Algorithm (RDA), Chirp-Scaling Algorithm 
(CSA), Omega-K Algorithm, and the SPECAN Algorithm [7]. Different algorithms provide different trade-offs between simplicity and efficiency. Details of these algorithms can be found in [7]. All of the algorithms perform three fundamental operations that are essential for SAR image formation:

- Range compression

- Range cell migration correction ( $\mathrm{RCMC})$

- Azimuth compression

Range compression involves a convolution of each received pulse with a pulse replica that has a conjugate quadratic phase. This pulse compression is also known as "matched filtering", since the filter is matched to the expected phase of the received signal. The purpose of range compression is to extract the target energy in the received pulse by removing the quadratic phase. After range compression, the received signal in Equation 2.9 can be written as 17

$$
s_{r}\left(t_{r}, t_{a}\right)=S^{\prime}\left(\alpha, t_{a}\right) p_{r}\left[t_{r}-\frac{2 R\left(t_{a}\right)}{\mathrm{c}}\right] w_{a}\left(t_{a}-t_{0}\right) \exp \left(-j 4 \pi \frac{R\left(t_{a}-t_{0}\right)}{\lambda}\right),
$$

where $p_{r}\left[t_{r}-\frac{2 R\left(t_{a}\right)}{\mathrm{c}}\right]$ is a delta-like range envelope that incorporates the range cell migration of the target, which is an artifact of the two-dimensional SAR data that results in the signal energy from a point target to follow a curved trajectory, which depends on the changing range delay to the target as it passes through the antenna beam during the target exposure time. $\mathrm{RCMC}$ is the process that corrects this curved trajectory. After $\mathrm{RCMC}$, the range envelope $p_{r}$ becomes independent of the azimuth varying parameter, $\frac{2 R\left(t_{a}\right)}{\mathrm{c}}$, and the signal can be written as 7

$$
s_{r}\left(t_{r}, t_{a}\right)=S^{\prime}\left(\alpha, t_{a}\right) p_{r}\left[t_{r}-\frac{2 R_{0}}{\mathrm{c}}\right] w_{a}\left(t_{a}-t_{0}\right) \exp \left(-j 4 \pi \frac{R\left(t_{a}\right)}{\lambda}\right) .
$$

Following RCMC, the signal is compressed in the azimuth direction by applying an azimuth matched filter, similar to the way the signal is compressed in the range direction

$$
s_{r}\left(t_{r}, t_{a}\right)=S^{\prime}\left(\alpha . t_{a}\right) p_{r}\left[t_{r}-\frac{2 R_{0}}{\mathrm{c}}\right] w_{a}\left(t_{a}\right) \exp \left(-j 4 \pi \frac{R_{0}}{\lambda}\right) \exp \left(j 2 \pi f_{t_{0}} t_{a}\right),
$$

where the second exponential term is due to the average Doppler frequency shift $f_{t_{0}}$ (Doppler centroid). Note that in the above form of the azimuth compressed signal, a parabolic model of the target-sensor range equation has been assumed. A hyperbolic 
model is typically used for high precision processing, which provides a better performance for non-zero squints [7]. After azimuth compression, the signal is focused in the azimuth direction.

Typically, when focusing SAR data, the azimuth compression filter is designed to register data to zero Doppler (i.e. to stationary clutter). However, this is not necessarily the case for SMTI. In fact, for SMTI, where the objective is to detect and estimate moving targets, the data is typically registered to the parameters of the moving target [1,2, 4, 19. This is discussed further in subsequent sections.

\subsection{Moving Target Signal Model}

In this section, a general signal model of the moving target for ISTAP is derived. This model will be used for all the subsequent analysis in this thesis. Note that a signal model for ISTAP has been presented in [14,20]. All the derivations have been performed in satellite earth-centered earth-fixed coordinate system (ECEF) coordinates, with the origin at the center of the Earth. In this coordinate system, the earth motion is absorbed into the relative satellite motion. Details about all the relevant coordinate systems are given in Appendix A.1.

\subsubsection{Range Equation}

The radar imaging geometry in satellite ECEF coordinate system is illustrated in Figure 2.3. In this coordinate system, the origin is at the centre of mass of earth. The x-axis is alligned in the satellite velocity direction. The z-axis is aligned along the vector from the centre of mass of earth to the center of mass of the satellite, and y-axis completes the right-handed coordinate system. More detailed discussion of the coordinate systems is provided in Appendix A. In the figure, $r_{s}$ denotes the distance from the center of mass of earth to the center of mass of the satellite, $r_{e}$ the radius of earth, and $h_{s}$ is the height of the satellite above the surface of earth. The incidence angle is denoted by $\theta$, and the satellite to point target range vector by $\mathbf{r}$. Note that all of these quantities are in satellite ECEF (D) coordinate system, which is defined in Appendix A. The range vector between the $\mathrm{n}$-th transmit/receive $(\mathrm{Tx} / \mathrm{Rx})$ antenna element and the moving target can be written as follows $2,7,19$

$$
\mathrm{r}=\mathrm{x}_{\mathrm{D}}^{n}-\mathrm{x}_{\mathrm{t}}
$$


where $\mathbf{x}_{\mathbf{D}}^{n}$ is the position vector for the $\mathrm{n}$-th $\mathrm{Rx} / \mathrm{Tx}$ antenna element and $\mathbf{x}_{\mathbf{t}}$ is the moving target position vector in satellite ECEF coordinate system. These position vectors are derived from earth and satellite geometry in Appendix A.1, and given as follows

$$
\begin{gathered}
\mathbf{x}_{\mathbf{D}}^{n}(t)=\left[\begin{array}{c}
V_{\mathrm{a}} t+r_{n} \\
0 \\
r_{s}-r_{n} \frac{a_{a}}{V_{\mathrm{a}}} t-\frac{a_{a}}{2} t^{2}
\end{array}\right] \\
\mathbf{x}_{\mathbf{t}}(t)=\left[\begin{array}{c}
V_{\mathrm{x}} t \\
y_{0}(\theta)+V_{y} t \\
z_{0}(\theta)+V_{z} t
\end{array}\right]
\end{gathered}
$$

where $V_{\mathrm{a}}$ is the satellite velocity, $r_{n}$ is the distance from the center of the satellite to the $n^{\text {th }}$ phase center, $a_{a}$ is the centripetal acceleration of the satellite that balances its gravitional acceleration, and $r_{s}$ is the distance from the center of the earth to the satellite. For a moving target, $y(\theta)$ and $z(\theta)$ are the incidence angle dependent initial coordinates along the $\hat{y}_{D}$ and $\hat{z}_{D}$ axis of the satellite ECEF coordinate system, $V_{\mathrm{x}}$ is the target alongtrack (along azimuth) velocity, $V_{y}$ and $V_{z}$ are the components of the target across-track (ground range) velocity along the $\hat{y}_{D}$ and $\hat{z}_{D}$ axis. 


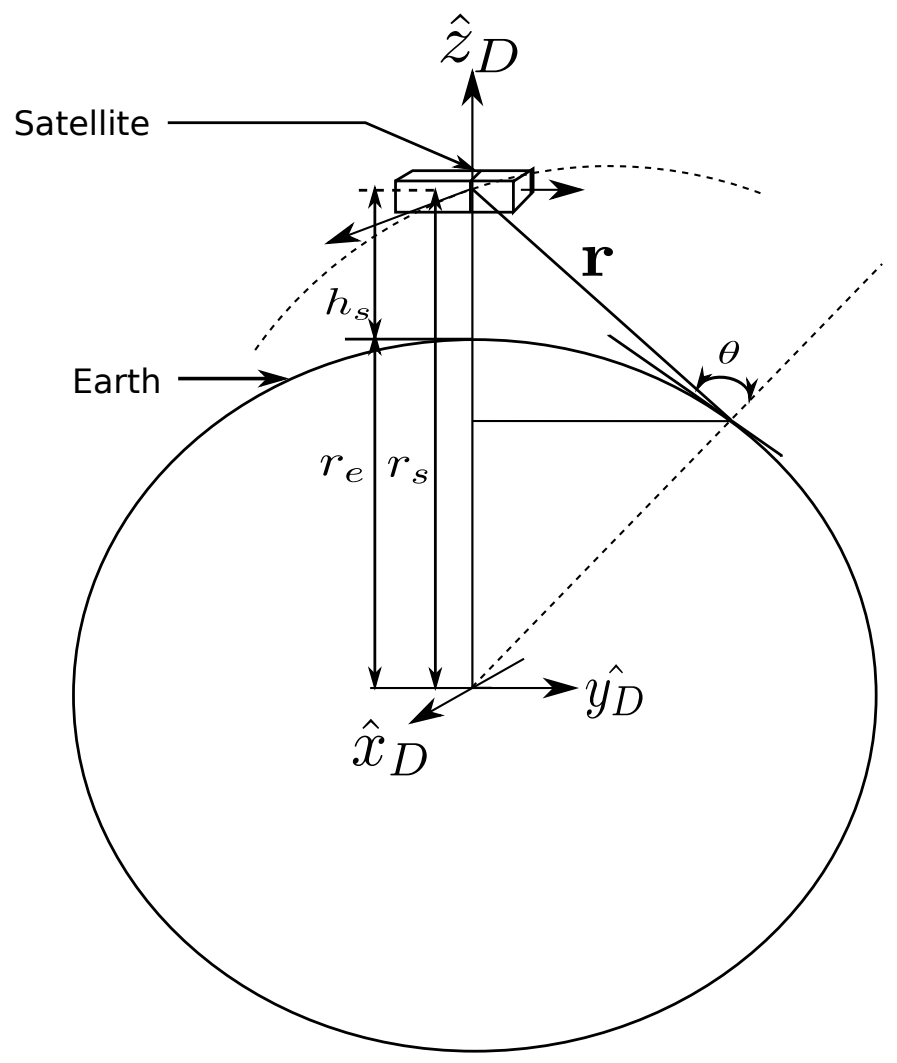

Figure 2.3: SAR imaging geometry in satellite ECEF coordinate system. The range vector, $\mathbf{r}$, is defined as the distance between the $n$-th transmit/receive $(\mathrm{Tx} / \mathrm{Rx})$ antenna element and the moving target. This vector is dependent on the azimuth time. The instantaneous range is a scalar quantity that is defined as the magnitude of the range vector. Incidence angle is represented by $\theta$. 
The instantaneous slant range, $R(t)$, which is the key parameter required for highprecision SAR processing, is defined as the magnitude of the vector $\mathbf{r}$ in Equation 2.13.

$$
R(t)=|\mathbf{r}|=\left|\mathbf{x}_{\mathbf{D}}^{n}-\mathbf{x}_{\mathbf{t}}\right|
$$

The instantaneous slant range equation is derived by substituting the position vectors of equations 2.14 and 2.15 in Equation 2.16 as

$$
\begin{aligned}
R(t) & =\sqrt{\left[\left(\left(V_{\mathrm{a}}-V_{\mathrm{x}}\right) t+r_{n}\right)^{2}+\left(V_{\mathrm{y}} t+y_{0}\right)^{2}+\left(r_{s}-z_{0}-\left(\frac{a_{a} r_{n}}{V_{\mathrm{a}}}+V_{\mathrm{z}}\right) t-\frac{1}{2} a_{a} t^{2}\right)^{2}\right]} \\
R^{2}(t) & =\left[\left(\left(V_{\mathrm{a}}-V_{\mathrm{x}}\right) t+r_{n}\right)^{2}+\left(V_{\mathrm{y}} t+y_{0}\right)^{2}+\left(r_{s}-z_{0}-\left(\frac{a_{a} r_{n}}{V_{\mathrm{a}}}+V_{\mathrm{z}}\right) t-\frac{1}{2} a_{a} t^{2}\right)^{2}\right]
\end{aligned}
$$

where $r_{s}$ is the distance from the center of the earth to the satellite, which according to the geometry of Figure 2.3 is determined as

$$
r_{s}=r_{e}+h_{s}
$$

The instantaneous range equation in $2.17 \mathrm{~b}$ can be approximated using a second-order Taylor series expansion

$$
\begin{array}{r}
R^{2}(t) \approx R_{i}^{2}\left(t_{0}\right)+R_{i}^{\prime}\left(t_{0}\right)^{2}\left(t-t_{0}\right)+\frac{R_{i}^{\prime \prime}\left(t_{0}\right)^{2}}{2}\left(t-t_{0}\right)^{2} \\
R^{2}(t) \approx c_{0}+c_{1} t+c_{2} t^{2}
\end{array}
$$

where Equation 2.19b is obtained by expanding the Taylor series expression around the reference time $t_{0}=0 . R^{\prime}\left(t_{0}\right)$ and $R^{\prime \prime}\left(t_{0}\right)$ denote the first and second-order derivatives at the reference time. The squared form of these derivatives, which are required in the Taylor series expansion of Equation 2.19a, are provided in equations 2.20 and 2.21 .

$$
\begin{gathered}
R^{\prime}(t)^{2}=2\left(\left(V_{\mathrm{a}}-V_{\mathrm{x}}\right) t+r_{n}\right)\left(V_{\mathrm{a}}-V_{\mathrm{x}}\right)+2\left(V_{y} t+y_{0}\right) V_{y}+ \\
2\left(r_{s}-z_{0}-\left(\frac{a_{a} r_{n}}{V_{\mathrm{a}}}+V_{z}\right) t-\frac{1}{2} a_{a} t^{2}\right) \\
\left(-\left(\frac{a_{a} r_{n}}{V_{\mathrm{a}}}+V_{z}\right)-a_{a} t\right) \\
R^{\prime \prime}(t)^{2}=2\left(V_{\mathrm{a}}-V_{\mathrm{x}}\right)^{2}+2 V_{y}^{2}+2\left[-\left(r_{s}-z_{0}\right) a_{a}+\left(\frac{a_{a} r_{n}}{V_{\mathrm{a}}}+V_{z}\right)^{2}+3 a_{a} t\left(\frac{a_{a} r_{n}}{V_{\mathrm{a}}}+V_{z}\right)+\frac{3}{2} a_{a}^{2} t^{2}\right]
\end{gathered}
$$


By using the expressions for the derivatives above and the assumptions $V_{\mathrm{a}} \gg a_{a}$ and $V_{\mathrm{a}} \gg r_{n}$, the constants in Equation 2.19b are derived as follows

$$
\begin{gathered}
c_{0}=\left[r_{n}^{2}+y_{0}^{2}+\left(r_{s}-z_{0}\right)^{2}\right] \\
c_{1}=2 \frac{r_{n}}{V_{\mathrm{a}}}\left(V_{a}^{2}-a_{a}\left(r_{s}-z_{0}\right)\right)-2 r_{n} V_{\mathrm{x}}+2 y_{0} V_{y}-2 V_{z}\left(r_{s}-z_{0}\right) \\
c_{2}=\left(V_{\mathrm{a}}-V_{\mathrm{x}}\right)^{2}+V_{y}^{2}+\left[\left(\frac{a_{a} r_{n}}{V_{\mathrm{a}}}+V_{z}\right)^{2}-a_{a}\left(r_{s}-z_{0}\right)\right] \\
=\left(V_{\mathrm{a}}-V_{\mathrm{x}}\right)^{2}+V_{y}^{2}+V_{z}^{2}-a_{a}\left(r_{s}-z_{0}\right)
\end{gathered}
$$

Equations $2.22-2.24 \mathrm{~b}$ can be further simplified by defining some useful quantities. The "effective radar velocity" ( $\left.V_{\text {eff }}\right)$ is an important parameter that is typically used in spaceborne SAR processing [7,21]. In spaceborne SAR, the satellite orbit is curved, the Earth's surface is curved, and the Earth rotates independently of the satellite orbit. The effective radar velocity is used to model this curved-geometry and provides an accurate representation of the range equation [7, 18].

$$
V_{\mathrm{eff}}=\sqrt{V_{\mathrm{a}}^{2}-a_{a}\left(r_{s}-z_{0}\right)}
$$

The relationship between the effective radar velocity $\left(V_{\text {eff }}\right)$, satellite velocity $\left(V_{\mathrm{a}}\right)$, and the ground velocity $\left(V_{\mathrm{g}}\right)$ is given as 7

$$
V_{\mathrm{g}} \approx \frac{V_{\mathrm{eff}}^{2}}{V_{\mathrm{a}}}
$$

The value of $V_{\text {eff }}$ varies with range and varies with azimuth due to the curvedgeometry. The numerical value of $V_{\text {eff }}$ lies between $V_{\mathrm{a}}$, which is the satellite platform velocity, and $V_{\mathrm{g}}$, which is the velocity of the radar beam as it moves along the ground. The approximation in Equation 2.26 is due to the fact that the satellite orbit is not perfectly circular [7]. Further details about the effective radar velocity can be found in 7, 18,21 .

Two other useful quantities that are often found in literature include the "relative radial velocity", which is the velocity between the moving target and the satellite, and the "target radial velocity", which is defined as the projection of the target velocity onto 
the line-of-sight (LOS) vector at $t=0$ [2, 4, 19]. The relative radial velocity is defined as follows

$$
\begin{aligned}
V_{r e l} & =\sqrt{\left(V_{\mathrm{x}}-V_{\mathrm{a}}\right)^{2}+V_{x^{\perp}}^{2}-a_{a}\left(r_{s}-z_{0}\right)} \\
& =\sqrt{V_{x}^{2}-2 V_{\mathrm{x}} V_{\mathrm{a}}+V_{a}^{2}+V_{x^{\perp}}^{2}-a_{a}\left(r_{s}-z_{0}\right)} \\
& =\sqrt{V_{e f f}^{2}+V_{x^{\perp}}^{2}+V_{x}^{2}-2 V_{\mathrm{x}} V_{\mathrm{a}}}
\end{aligned}
$$

where $V_{\mathrm{x}}^{\perp}$ is the target across-track velocity. The expression for target radial velocity is explicitly derived from the imaging geometry in Appendix A.4, and is given as follows

$$
V_{r t}^{\perp}=V_{\mathrm{x}}^{\perp} \sin (\theta)
$$

where $\theta$ is the incidence angle (see Appendix A.4.

By using the terms defined in equations 2.25 2.28, the coefficients $c_{0}, c_{1}$, and $c_{2}$ can be written as

$$
\begin{gathered}
c_{0}=R_{0}^{2}+r_{n}^{2} \\
c_{1}=2 r_{n}\left(V_{\mathrm{g}}-V_{\mathrm{x}}\right)+2 R_{0} V_{r t}^{\perp} \\
c_{2}=V_{r e l}^{2}
\end{gathered}
$$

where $R_{0}$ is defined as

$$
R_{0}=\sqrt{\left(r_{e}+h_{s}-z_{0}\right)^{2}+y_{0}^{2}}=\sqrt{\left(r_{s}-z_{0}\right)^{2}+y_{0}^{2}} .
$$

The final instantaneous range equation is then given as

$$
R_{n}^{2}(t)=\left(R_{0}^{2}+r_{n}^{2}\right)+2\left(\left(V_{\mathrm{g}}-V_{\mathrm{x}}\right) r_{n}+R_{0} V_{r t}^{\perp}\right) t+V_{r e l}^{2} t^{2}
$$

\subsubsection{Antenna Array Pattern}

In this section, an expression for the antenna array pattern is derived as a function of the antenna look-direction and the multi-channel switching and toggling [13]. The antenna look-direction is defined as the direction along which the radar pulse travels in the Antenna Coordinate System (System A). An expression for the antenna lookdirection has been derived in Appendix A.4.1 and is explicitly given in Equation A.32. The two-way antenna pattern is the product of the one-way transmit pattern and the one-way receive pattern. The one-way antenna pattern is modeled as the product of the complex beamforming vector and the direction of arrival vector, $\left(\vec{d}\left(u_{t}\right)\right)$. For an 
antenna with $N$ transmit-receive columns, the complex beamforming vector is given by $\mathbf{w}_{(t, r)} \in \mathbb{C}^{N \times 1}$, where the subscripts " $\mathrm{t}$ " and " $\mathrm{r}$ " stand for "transmit" and "receive", respectively. Such representation of the complex beamforming vector incorporates the individual transmit and receive antenna configuration for each column, and therefore it completely describes antenna switching/toggling characteristics [4,13,20]. The M-channel antenna array pattern that incorporates switching/toggling configuration is then written as

$$
\mathbf{A}\left(u_{t}\right)=\left[\begin{array}{c}
\left(\mathbf{w}_{t, 1}^{\dagger} \mathbf{d}\left(u_{t}\right)\right) \cdot\left(\mathbf{w}_{r, 1}^{\dagger} \mathbf{d}\left(u_{t}\right)\right) \\
\left(\mathbf{w}_{t, 2}^{\dagger} \mathbf{d}\left(u_{t}\right)\right) \cdot\left(\mathbf{w}_{r, 2}^{\dagger} \mathbf{d}\left(u_{t}\right)\right) \\
\vdots \\
\left(\mathbf{w}_{t, M}^{\dagger} \mathbf{d}\left(u_{t}\right)\right) \cdot\left(\mathbf{w}_{r, M}^{\dagger} \mathbf{d}\left(u_{t}\right)\right)
\end{array}\right]
$$

where $\dagger$ is the conjugate transpose operator, and $\mathbf{d}\left(u_{t}\right)$ is the direction-of-arrival (DOA) vector 13,20

$$
\mathbf{d}\left(u_{t}\right)=E_{e}\left(u_{t}\right)\left[\begin{array}{c}
\exp \left(-j \beta x_{1} u_{t}\right) \\
\exp \left(-j \beta x_{2} u_{t}\right) \\
\vdots \\
\exp \left(-j \beta x_{N} u_{t}\right)
\end{array}\right]
$$

where $E_{e}\left(u_{t}\right)$ denotes the elemental factor of the array antenna, $\beta=\frac{2 \pi}{\lambda}$ is the angular wavenumber, $x_{n}$ is the distance from the $n^{\text {th }}$ element to the antenna center with origin at antenna center, and $u_{t}$ is the first order Taylor approximation of the azimuth angle directional cosine of the look-direction vector or simply "look direction" [2,13]. This has been derived from the satellite-earth geometry in Section A.4.1 in Appendix A and is as follows

$$
u_{t}(t) \approx \frac{V_{\mathrm{x}}-V_{\mathrm{g}}}{R_{0}}(t)
$$

Note that the subscript "t" in the above equation denotes that the look direction is for a moving target. The look-direction for stationary clutter, which is used to define standard azimuth parameters, but not used in the signal model, is given as follows 7

$$
u_{c}(t) \approx \frac{-V_{\mathrm{g}}}{R_{0}}(t)
$$

where the subscript "c" denotes the look direction for stationary clutter. 


\subsubsection{Signal in Doppler Domain}

The two-dimensional signal model after range compression, which is given in Equation 2.10, will be used as the starting point in the derivation of the multi-channel signal model for ISTAP. However, the following simplifying assumptions can be made without affecting the analysis of the problem under consideration.

- The point-target signal at the peak of its range response after range compression will be considered. Under this assumption, the range pulse envelope $\left(p_{r}\left[\tau-\frac{2 R(\eta)}{c}\right]\right)$ is considered to be unity and can be ignored without any effect on the analysis of the problem under consideration. Due to this assumption, the signal of Equation 2.10 becomes invariant in the range direction and only the signal in the azimuth direction is considered [2,3].

- The complex function, $S^{\prime}(\alpha, \eta)$, which models the target reflectivity and the constant phase change due to the scatter can be written as follows [2]

$$
S^{\prime}(\alpha, \eta)=\sigma_{s} \exp \left(j \Delta_{s}\right)
$$

where $\sigma_{s}$ is some unknown amplitude and $\Delta_{s}$ is some unknown phase. For convenience, this term will be ignored when deriving the signal in the Doppler domain. This can be done since the term is independant of time $t$. However, the signal power depends on this term and, therefore, it can't be ignored when computing CRLB. This term will be later added to the final target signal model.

By using the phased-array antenna pattern of Equation 2.34 and the assumptions above, the time domain received signal of Equation 2.10 from a point-target in azimuth time, centered at $t_{0}$, and at the peak of its range response can be written as $[1-4$

$$
\mathbf{S}_{r}(t)=\mathbf{A}\left(u\left(t-t_{0}\right)\right) \cdot \exp \left(-2 j \beta R\left(t-t_{0}\right)\right)
$$

Note that the azimuth antenna gain pattern in Equation 2.10, $\omega_{a}\left(t-t_{0}\right)$, has been replaced with the phased array antenna pattern $\mathbf{A}(u(t))$. Also note that the $t$ in the above and all subsequent equations refers to azimuth time due to the first assumption 
noted above. The Fourier transform of the signal is given by

$$
\begin{aligned}
\mathbf{S}_{r}(\omega) & =\int \mathbf{A}\left(u\left(t-t_{0}\right)\right) \exp \left(-2 j \beta R\left(t-t_{0}\right)\right) \exp (-j \omega t) d t \\
& =\exp \left(-j \omega t_{0}\right) \int \mathbf{A}(u(t)) \exp \left(-2 j \beta \sqrt{c_{0}+c_{1} t+c_{2} t^{2}}-j \omega t\right) d t \\
& =\exp \left(-j \omega t_{0}\right) \int \mathbf{A}(u(t)) \exp \left(-2 j\left(\beta \sqrt{\left.\left.c_{2}\left(t+\frac{c_{1}}{2 c_{2}}\right)^{2}+c_{0}-\frac{c_{1}^{2}}{4 c_{2}}-\omega t\right)\right) d t}\right.\right. \\
& =\exp \left(-j \omega t_{0}\right) \exp \left(j \omega \frac{c_{1}}{2 c_{2}}\right) \int \mathbf{A}\left(u\left(t-\frac{c_{1}}{2 c_{2}}\right)\right) \exp \left(-2 j\left(\beta \sqrt{c_{2} t^{2}+C}-\omega t\right)\right) d t \\
& =\exp \left(j \omega\left(\frac{c_{1}}{2 c_{2}}-t_{0}\right)\right) \int \mathbf{A}\left(u\left(t-\frac{c_{1}}{2 c_{2}}\right)\right) \exp \left(-2 j\left(\beta \sqrt{c_{2} t^{2}+C}-\omega t\right)\right) d t
\end{aligned}
$$

where $C=c_{0}-\frac{c_{1}^{2}}{4 c_{2}}$. At this point, further derivation is not straightforward. An approximation can be conveniently obtained by applying the Principle of Stationary Phase (POSP) [7]. This principle is based on the fact that under certain conditions, the contribution to the integral lies mainly around the point when the phase is "stationary". This stationary phase point is determined as follows. The phase of the signal, $g(t)$, is given in Equation 2.41. This phase is considered to be stationary when the time derivative, $\frac{d g}{d t}$, is equal to zero. Around this point, the phase and the amplitude are varying slowly. A complete derivation using POSP is given in Appendix $\mathrm{C}$ and the result of Equation C.6b will be used in the subsequent derivation.

$$
g(t)=-2 \beta \sqrt{c_{2} t^{2}+C}-\omega t
$$

The stationary phase point is derived as

$$
\begin{array}{r}
\frac{d g}{d t}=\frac{-2 \beta c_{2} t}{\sqrt{c_{2} t^{2}+C}}-\omega=0 \\
\sqrt{c_{2} t_{s}^{2}+C}=-\frac{2 \beta c_{2} t_{s}}{\omega} \\
t_{s}= \pm \sqrt{\frac{\omega^{2} C}{c_{2}\left(4 \beta^{2} c_{2}-\omega^{2}\right)}} \\
t_{s}=-\frac{\omega}{2 \beta c_{2}} \frac{\sqrt{C}}{\sqrt{1-\frac{\omega^{2}}{4 \beta^{2} c_{2}}}}
\end{array}
$$


where $t_{s}$ is the time at the stationary point and the negative sign has been adopted in Equation 2.42d. The positive stationary point will yield the same expression for the Fourier transform but with a conjugate phase. The subsequent analysis will not be affected by the adopted convention. The phase at this point is derived as

$$
\begin{aligned}
g\left(t_{s}\right) & =-2 \beta\left(\frac{-2 \beta c_{2} t_{s}}{\omega}\right)-\omega t_{s} \\
& =\frac{4 \beta^{2} c_{2}-\omega^{2}}{\omega} t_{s} \\
& =-2 \beta \sqrt{C} \sqrt{1-\frac{\omega^{2}}{4 \beta^{2} c_{2}}} .
\end{aligned}
$$

The second derivative of the phase term is derived as

$$
\begin{aligned}
\frac{d^{2} g(t)}{d t^{2}} & =\frac{-2 \beta c_{2} \sqrt{c_{2} t^{2}+C}-\left(c_{2} t^{2}+C\right)^{-1 / 2} c_{2} t\left(-2 \beta c_{2} t\right)}{c_{2} t^{2}+C} \\
& =\frac{-2 \beta c_{2}}{\left(c_{2} t^{2}+C\right)^{1 / 2}}+\frac{\left(2 \beta c_{2} t\right)^{3}}{\left(c_{2} t^{2}+C\right)^{3 / 2}} \frac{1}{(2 \beta)^{2} c_{2} t} .
\end{aligned}
$$

Substituting the term for stationary point from Equation 2.42d into Equation 2.44b yields

$$
\begin{aligned}
\left.\frac{d^{2} g(t)}{d t^{2}}\right|_{t=t_{s}} & =\frac{\omega}{t_{s}}-\frac{\omega^{3}}{(2 \beta)^{2} c_{2} t_{s}} \\
& =\frac{2 \beta c_{2}}{\sqrt{C}}\left(1-\frac{\omega^{2}}{4 \beta^{2} c_{2}}\right) \\
& \approx \frac{4 \pi V_{r e l}^{2}}{R_{0} \lambda}
\end{aligned}
$$

where the approximation is because it is assumed that $V_{\mathrm{x}}^{\perp}<<V_{\text {rel }}$ and $\omega<<V_{\text {rel }}$. Note that these assumptions are only valid for $2.45 \mathrm{c}$ because this term is in the amplitude of the signal. Similar to far-field approximation (see Appendix B), these assumptions cannot be applied in the exponential phase terms.

The signal model in the Doppler domain is obtained by substituting equations $2.42 \mathrm{~d}$, $2.44 \mathrm{~b}, 2.40 \mathrm{e}$ into the stationary phase approximation result of Equation C.6b

$$
\begin{array}{r}
\mathbf{S}_{r}(\omega) \approx \sqrt{\frac{R_{0} \lambda}{j 2 V_{r e l}^{2}}} \exp \left(j \omega\left(\frac{c_{1}}{2 c_{2}}-t_{0}\right)\right) \mathbf{A}\left(u\left(-\frac{\omega}{2 \beta c_{2}} \frac{\sqrt{C}}{\sqrt{1-\frac{\omega^{2}}{4 \beta^{2} c_{2}}}}-\frac{c_{1}}{2 c_{2}}\right)\right) \\
\exp \left(-2 j \beta \sqrt{C} \sqrt{1-\frac{\omega^{2}}{2 \beta^{2} c_{2}}}\right) .
\end{array}
$$


The expression for the signal model in Equation 2.46 can be re-written by substituting the values of the constants:

$$
\begin{array}{r}
\mathbf{S}_{r}(\omega)=\sqrt{\frac{R_{0} \lambda}{j 2 V_{r e l}^{2}}} \exp \left(j \omega\left(\frac{R_{0} V_{r t}^{\perp}}{2 V_{r e l}^{2}}-t_{0}\right)\right) \mathbf{A}\left(u\left(\frac{-\omega R_{0}}{2 \beta V_{r e l}^{2}} \sqrt{\frac{V_{r e l}^{2}+4 V_{r t}^{\perp}}{4 \beta^{2} V_{r e l}^{2}-\omega^{2}}}-\frac{R_{0} V_{r t}^{\perp}}{2 V_{r e l}^{2}}\right)\right) \\
\exp \left(-2 j \beta R_{0} \sqrt{1-\left(\frac{V_{r t}^{\perp}}{V_{r e l}}\right)^{2}} \sqrt{1-\frac{(\omega)^{2}}{4 \beta^{2} V_{r e l}^{2}}}\right) .
\end{array}
$$

Note that for simplicity all the small terms containing $r_{n}$ have been ignored. This can be justified since the terms containing $r_{n}$ are at least two orders of magnitude smaller than the other terms and can be neglected in the range equation without significantly affecting the accuracy of the results. By using Equation 2.36, the frequency domain directional cosine term $u(\omega)$ is expressed as

$$
u(\omega) \approx \frac{\left(V_{g}-V_{x}\right)}{V_{r e l}^{2}}\left(\frac{\omega}{2 \beta} \sqrt{\frac{1-\left(\frac{V_{r t}^{\perp}}{V_{r e l}}\right)^{2}}{1-\left(\frac{\omega}{2 \beta V_{r e l}}\right)^{2}}}+V_{r t}^{\perp}\right) .
$$

The azimuth sampling of the signal at pulse repetition frequency $\left(w_{p}\right)$ and the target radar cross section ( $(\underline{\mathrm{RCS}})$ dependant phase and amplitude of Equation 2.38 must be taken into account. It is more convenient to incorporate this in the single channel expression of the signal model and then write the multi-channel model as a vector. The single channel signal model that incorporates the PRF sampling and target RCS is written as

$$
\begin{array}{r}
\mathbf{S}_{r, M}(\omega)=\sigma_{s} \exp \left(j \Delta_{s}\right)\left(\sqrt { \frac { R _ { 0 } \lambda } { j 2 V _ { r e l } ^ { 2 } } } \sum _ { k } \left\{\left(\mathbf{w}_{t, M}^{\dagger} \mathbf{d}\left(u\left(\omega+k \omega_{p}\right)\right)\right) \cdot\left(\mathbf{w}_{r, M}^{\dagger} \mathbf{d}\left(u\left(\omega+k \omega_{p}\right)\right)\right)\right.\right. \\
\exp \left(-j\left(\omega+k \omega_{p}\right) \delta_{M}\right) \exp \left(j\left(\omega+k \omega_{p}\right)\left(\frac{R_{0} V_{r t}^{\perp}}{2 V_{r e l}^{2}}-t_{0}\right)\right) \\
\left.\left.\exp \left(-2 j \beta R_{0} \sqrt{1-\left(\frac{V_{r t}^{\perp}}{V_{r e l}}\right)^{2}} \sqrt{1-\frac{\left(\omega+k \omega_{p}\right)^{2}}{4 \beta^{2} V_{r e l}^{2}}}\right)\right\}\right) .
\end{array}
$$

where $\delta_{M}$ is the sampling delay for the $M$ th channel, $\omega_{p}$ is the pulse-repetition frequency, and the subscript $M$ denotes that this is the signal from the $M$ th channel. The multi- 
channel signal model is then written as a vector as $[1-4]$

$$
\mathbf{S}_{r}(\omega)=\left[\begin{array}{c}
\mathbf{S}_{r, 1} \\
\mathbf{S}_{r, 2} \\
\vdots \\
\mathbf{S}_{r, M}
\end{array}\right]
$$

\subsubsection{Azimuth Parameters}

As discussed in the preceding section, the SAR signal is also linearly modulated in frequency in the azimuth dimension. This is in fact the defining characteristic of SAR 7, 18, 21,22. In this section, some of the key azimuth parameters are derived for moving and, where necessary, stationary targets. The parameters for moving targets are denoted by superscript "m" and parameters for stationary clutter targets are denoted by superscript "c". Azimuth parameters depend on the instantaneous range, which is defined in Equation 2.33, and the instantaneous velocity, which is found by taking the first-order derivative of the instantaneous range as

$$
\begin{aligned}
V_{\mathrm{in}}^{m}\left(t_{a}\right) & =\frac{\mathrm{d} R\left(t_{a}\right)}{\mathrm{d} t_{a}}=\frac{\mathrm{d}\left(\sqrt{\left(V_{\mathrm{rel}} t_{a}\right)^{2}+2 R_{0} V_{\mathrm{rt}}^{\perp}+R_{0}^{2}}\right)}{\mathrm{d} t_{a}} \\
& =\frac{V_{\mathrm{rel}}^{2} t_{a}+R_{0} V_{\mathrm{rt}}^{\perp}}{R_{n}\left(t_{a}\right)}
\end{aligned}
$$

where it has been assumed that all other velocities are constant with respect to azimuth time. Note that the expression for instantaneous velocity has been derived with respect to the center of the antenna $\left(r_{n}=0\right)$. It is often useful to write $V_{\text {in }}\left(t_{a}\right)$ in terms of the target look direction of Equation A.31. The instantaneous velocity for moving target in terms of the look direction is given by

$$
V_{\mathrm{in}}^{m}\left(t_{a}\right)=-\frac{V_{\mathrm{rel}}^{2}}{V_{\mathrm{g}}-V_{\mathrm{x}}} u_{t}\left(t_{a}\right)+V_{\mathrm{rt}}^{\perp} .
$$

The rate of change for stationary target (clutter) range is derived by setting all the target dependent velocities in Equation 2.52 to zero

$$
V_{\text {in }}^{c}\left(t_{a}\right)=-V_{\mathrm{a}} u_{c}\left(t_{a}\right)
$$

where $u_{c}\left(t_{a}\right)$ is the look direction for the clutter and is given by

$$
u_{c}\left(t_{a}\right)=-\frac{V_{\mathrm{g}} t_{a}}{R_{c}\left(t_{a}\right)}
$$

where $R_{c}\left(t_{a}\right)$ is the range to a stationary target. 


\section{Doppler Frequency}

The Doppler frequency is proportional to the rate of change of $R\left(t_{a}\right)$

$$
f_{d}\left(t_{a}\right)=-\frac{2}{\lambda} \frac{\mathrm{d} R\left(t_{a}\right)}{\mathrm{d} t_{a}}
$$

where $\lambda$ is the center wavelength of the radar. The Doppler frequency is given in units of Hertz. Note the negative slope of the Doppler frequency that was discussed in Section 2.1.3.

The Doppler frequency with respect to a moving target and clutter are derived using equations 2.52 and 2.53 respectively.

$$
\begin{aligned}
& f_{d}^{m}\left(t_{a}\right)=-\frac{2}{\lambda} V_{\mathrm{in}}^{m}\left(t_{a}\right) \\
& f_{d}^{c}\left(t_{a}\right)=-\frac{2}{\lambda} V_{\mathrm{in}}^{c}\left(t_{a}\right) .
\end{aligned}
$$

\section{Doppler Bandwith}

The Doppler bandwidth is the frequency excursion experienced by a point target during the time it is illuminated by the $3-\mathrm{dB}$ beamwidth of the real aperture

$$
\Delta f_{d}=f_{d, \text { high }}-f_{d, l o w}=-\left.\frac{2}{\lambda} \frac{\mathrm{d} R\left(t_{a}\right)}{\mathrm{d} t_{a}}\right|_{t_{a}=T_{a}},
$$

where $T_{a}$ is the target exposure time or dwell time, which is the duration the target stays in the $3-\mathrm{dB}$ beam limits. The value of $T_{a}$ is derived in Appendix $\mathrm{B}$ from the scene geometry and is given as

$$
T_{a}=0.886 \frac{\lambda R\left(t_{a, c}\right)}{L_{a} V_{\mathrm{g}}}
$$

Using this expression, the bandwidth for stationary clutter is as

$$
\Delta f_{d}^{c}=0.886 \frac{2 V_{\mathrm{a}}}{L_{a}}
$$

The bandwidth for a moving target can be derived in a similar manner.

The Doppler bandwidth puts constraints on the lower limit of the PRF. More specifically, in order satisfy the Nyquist criterion the PRF must be greater than the Doppler bandwidth

$$
\Delta f_{d}<f_{p}
$$

where $f_{p}$ is the PRF. Typically, an oversampling factor of 1.1 to 1.4 is used to reduce the azimuth ambiguity power [7]. 


\section{Azimuth Resolution}

The time resolution that can be obtained is 0.886 times the reciprocal of the bandwidth. To convert the resolution in distance units, the time resolution can be multiplied by the beam velocity $V_{\mathrm{g}}$ to give

$$
\rho=\frac{0.886 V_{\mathrm{g}}}{\Delta f_{d}}=\frac{L_{a}}{2} \frac{V_{\mathrm{g}}}{V_{\mathrm{a}}}
$$

The above expression shows that the azimuth resolution is approximately one-half the antenna length and is independant of the range or wavelength.

\subsection{Methods for Surface Moving Target Indication}

SAR was originally developed and traditionally used for imaging a stationary surface. As a result, moving targets appear smeared and defocused in a SAR image. Traditional SAR based moving target indication (MTI) systems, which employ only a single channel, are only able to detect moving targets that move sufficiently fast such that their Doppler shifts lie outside the stationary clutter bandwidth [23]. The Doppler shift is a function of the relative radial velocity between the radar and the target. Due to this limitation, single-channel SAR systems are incapable of detecting slow moving targets. Therefore, array antenna methods that employ mutilple channels are typically used to provide satisfactory performance.

In radar literature, the terms "Ground Moving Target Indication (GMTI)" and "Surface Moving Target Indication (SMTI)" are typically used to refer to multi-channel array techniques to detect and estimate moving targets, whereas the term "Moving Target Indication (MTI)" is used to refer to radar systems that use a single channel for target detection [24]. In this section, multi-channel SAR concepts are first presented in Subsection 2.3.1. Conventional methods for SAR based SMTI, which include along-track interferometry (ATI), displaced phase center antennas (displaced phase center antenna (DPCA)), and imaging space-time adaptive processing (ISTAP) are discussed in subsections 2.3.2, 2.3.3, and 2.3 .4 respectively.

\subsubsection{Multi-channel SAR SMTI}

In traditional (single-channel) SAR systems, as the platform advances along in the azimuth direction, a single antenna is used to take a single image of the ground at each time instant $t_{a}$. As a result, a single snapshot of the scene is produced during the entire 
azimuth interval. In contrast, multi-channel SAR SMTI systems use multiple antennas to image the same portion of the ground at multiple time intervals. For example, a 2-channel SAR SMT] system employs two antennas to image a section of the ground at time $t_{a}$ and $t_{a}+\Delta t_{a}$, where the displacement time $\Delta t_{a}$ is given by the speed of the platform and the spatial separation, or baseline, between the antennas. In this manner, multi-channel SAR SMTI systems can take multiple snapshots at the same spatial position, but varying temporal position. This allows for greater degrees of freedom for detection and estimation, which significantly improves the SMTI performance [5,24, 25].

Many different configurations can be employed to create or synthesize multiple channels in SAR systems. In the simplest configuration, a single transmit antenna and two receive antennas are placed strategically along the flight path of the radar. In this manner, samples can be received at phase-centers that are typically within a few milliseconds of each other. Responses from stationary reflectors (i.e., clutter) from the two phase-centers are highly-correlated and can be distinguished from the responses from moving target, which exhibit a significant phase difference. In air-borne SAR systems, multiple phase centers are typically created using multiple physical transmit or receive channels. In the case of space-borne SAR systems, the number of physical channels are limited due to the large weight, power consumption, and data-rate restrictions. Canadian RADARSAT-2 and German TerraSAR-X satellites, which are the only two unclassified satellites that are capable of performing space-borne SMTI, have only two physical channels. However, these satellites are equipped with a controllable phased-array antenna, which can be used to synthesize virtual channels by strategically switching and toggling the antenna sub-apertures [13,20]. In the context of this report, toggling refers to the pulse-to-pulse activation and deactivation of parts of the transmit aperture and switching refers to the pulse-to-pulse activation and deactivation of the parts of the receive aperture.

As mentioned previously, the RADARSAT-2 is equipped with two physical channels for signal acquisition and a controllable phased array antenna [26, 27]. The antenna consists of 512 transmit-receive (TR) modules. The planar phased array is constructed using 16 columns, each with 32 independently controllable TR modules, which allow beam shaping and steering, as well as on-off switching of entire columns. This enables the radar to operate under a set of switching/toggling schemes that have been specifically developed for space-borne GMTI and are collectively known as Moving Object Detection Experiment (MODEX) 3. 19.28. A set of MODEX modes are presented in detail in [19. A subset of these modes are discussed in Appendix $D$. 


\subsubsection{Along-Track Interferometry}

Along-Track Interferometry (ATI) is a popular approach that has been adapted for spaceborne SAR SMTI 29, 30]. In the simplest case, two measurements of the same scene separated by a short time interval are used to form an interferogram by taking the conjugate product. The phase of this product, which is called along-track interferometry (ATI) phase, provides a measurement of the radial velocity of the scatterers [29]. Under ideal conditions, stationary targets (clutter) don't show any ATI phase (i.e., zero ATI phase), whereas moving targets exhibit a non-zero ATI phase. In reality, however, the clutter usually exhibits a non-zero phase distribution, but moving targets can still be detected by applying a statistical "Constant False Alarm Rate (CFAR)", which essentially uses a phase threshold value for detections. The threshold value is chosen based on a clutter model and the desired false alarm rate. A detailed discussion of the constant false alarm rate (CFAR) test can be found in [31,32].

More precisely, ATI consists of at least two measurements from two identical scenes that are $\Delta t_{a}$ apart. Let these two measurements be denoted by $z_{1}\left(t_{a}\right)$ and $z_{2}\left(t_{a}\right)$ as 30,33

$$
\begin{array}{r}
z_{1}\left(t_{a}\right)=A\left(t_{a}\right) \exp \left(-j \frac{2 \pi}{\lambda} 2 R\left(t_{a}\right)\right), \\
z_{2}\left(t_{a}\right)=A\left(t_{a}+\Delta t_{a}\right) \exp \left(-j \frac{2 \pi}{\lambda} 2 R\left(t_{a}+\Delta t_{a}\right)\right),
\end{array}
$$

where $A$ is the amplitude and $R\left(t_{a}\right)$ is the range to the target at azimuth time $t_{a}$. An interferogram is formed by taking the conjugate product of the two measurements as $30,33,34$

$$
z_{1} * z_{2}\left(t_{a}\right)=\sum_{m=0}^{n-1}\left(\frac{z_{1}(m) * z_{2}(m)}{\sqrt{\left|z_{1}(m)\right|^{2}\left|z_{2}(m)\right|^{2}}}\right)
$$

where $n$ is the total number of azimuth samples.

The ATI phase is computed from the argument of the quantity in Equation 2.64. For detection of moving targets, the ATI phase is modelled as a random variable with zero mean for stationary clutter. The variance of the phase defines the limit on the minimum detectable velocity. The phase threshold for CFAR detection is computed analytically using the ATI phase statistics [32].

\subsubsection{Extended Displaced Phase Center Antenna}

Another approach to target detection is known as Displaced Phase Center Antenna (DPCA), which is quite similar to ATI, in that the detection is performed by measuring 
the difference between measurements from antennas with phase centers that are displaced in along-track 2, 9, 33. The measurements can be taken the same way as discussed in Section 2.3.2, but instead of measuring the cross-correlation as in Equation 2.64, the difference between the $m-t h$ azimuth samples is taken as $2,4,9,35$

$$
m_{D P C A}=\sum_{m=0}^{n-1}\left|z_{1}(m)-z_{2}(m)\right|^{2} .
$$

Under ideal conditions, the difference between the measurements of stationary targets should be zero, and the difference between the measurements of non-stationary (i.e., moving) targets should be non-zero [33].

Note that the DPCA method of Equation 2.65 is applicable for only two-channel SAR systems. An extension of the DPCA concept to more than two channels, which is appropriately called "Extended DPCA (EDPCA)", has been derived in [35]. Before discussing EDPCA, it is important to familiarize with the basic concepts of statistical testing in the context of SARISMTI. In traditional radar detection systems, the detection of target is performed using a statistical generalized likelihood ratio test GLRT, which requires a statistical ("likelihood") model for null hypothesis, and similar model for an alternative hypothesis [16]. In the context of SAR SMTI, the hypotheses are stated as follows

$$
\begin{array}{r}
\mathrm{H}_{0}: \mathbf{Z}=\mathbf{C}+\mathbf{N} \\
\mathrm{H}_{1}: \mathbf{Z}=\mathbf{C}+\mathbf{N}+\mathbf{s}(\Theta)
\end{array}
$$

where $\mathbf{Z}=\left(Z_{1}, Z_{2}, \ldots, Z_{M}\right)^{T}$ is the received signal vector, consisting of $M$ channels, $\mathbf{C}$ is the clutter signal, $\mathbf{N}$ is the noise signal, and $\mathbf{s}(\Theta)$ is the moving target signal, which is a function of the moving target parameter set $\Theta$, which vary depending on the signal model under consideration. The null-hypothesis $\left(\mathrm{H}_{0}\right)$ states that the signal does not contain the moving target and the alternate-hypothesis $\left(\mathrm{H}_{1}\right)$ states that the signal contains the moving target. The generalized likelihood ratio test (GLRT) is then stated as

$$
\frac{p_{\mathbf{z}}\left(\mathbf{Z} \mid \mathrm{H}_{1}\right)}{p_{\mathbf{z}}\left(\mathbf{Z} \mid \mathrm{H}_{0}\right)} \stackrel{\mathrm{H}_{1}}{\gtrless} T_{\Lambda}
$$

where $p_{\mathbf{z}}\left(\mathbf{Z} \mid H_{1}\right)$ is the probability density function (PDF) of signal given that the target was present, $p_{\mathbf{z}}\left(\mathbf{Z} \mid H_{0}\right)$ the PDF given the target was not present, and $T_{\Lambda}$ is the CFAR threshold value, which controls the acceptable amount of false alarms [16. In the context 
of GLRT, the left-side of Equation 2.67 is called the "likelihood ratio" or "test statistic". A typical radar detection system computes a test statistic and compares it to a threshold value, which is selected based on desireable amount of probability of false alarm [16]. If the value of the likelihood ratio is greater than the threshold value, then $\mathrm{H}_{1}$ is choosen. If the likelihood ratio does not exceed the threshold value, then the radar processor selects $\mathrm{H}_{0}$, which states that a moving target is not present in the signal.

According to Equation 2.67, in order to derive a test statistic for EDPCA, the probability density functions for the hypotheses stated in equations 2.66a and 2.66b must be first defined. A statistical model for the clutter-plus-noise interference signal, which is the received signal according the null hypothesis, can be derived by assuming that the interference is spatially statistically independent and zero mean complex Gaussian distributed. This is a valid assumption for the problem under consideration and many other applications $14,35,36$. Using this assumption, the PDF for the clutter-plus-noiseinterference (null-hypothesis) for EDPCA is given as 35]

$$
f_{\mathbf{Z}\left(t_{r}, t_{a}, \Theta\right)}(\mathbf{Z})=\frac{1}{\pi^{M}\left|\boldsymbol{\Sigma}_{\mathbf{E}}(\Theta)\right|} \mathrm{e}^{\left(-\mathbf{Z}^{\dagger} \boldsymbol{\Sigma}_{\mathbf{E}}(\Theta)^{-1} \mathbf{Z}\right)}
$$

where $\mathbf{Z}$ is the signal model as defined in Equation 2.66a, and $\boldsymbol{\Sigma}_{\mathbf{E}}$ is the clutter-plus-noise covariance matrix for EDPCA, which is estimated by averaging over a set of range and azimuth samples

$$
\boldsymbol{\Sigma}_{\mathbf{E}}=\frac{1}{N_{r} N_{a}} \sum_{t_{r} \in \Omega_{r}} \sum_{t_{a} \in \Omega_{a}} \mathbf{Z}\left(t_{r}, t_{a}, \Theta\right) \mathbf{Z}^{\dagger}\left(t_{r}, t_{a}, \Theta\right)
$$

where $\Omega_{r}$ and $\Omega_{a}$ are the range and azimuth sets over which the averaging is performed, and $N_{r}$ and $N_{a}$ is the cardinality of the sets.

The PDF for the alternate hypothesis of Equation $2.66 \mathrm{~b}$ is derived by noting that moving target model $\mathbf{s}(\Theta)$ is deterministic, and hence can be added to the Gaussian model as

$$
f_{\mathbf{Z}\left(t_{r}, t_{a}, \Theta\right)}(\mathbf{Z})=\frac{1}{\pi^{M}\left|\mathbf{\Sigma}_{\mathbf{E}}(\Theta)\right|} \mathrm{e}^{-\left[\mathbf{Z}+\mathbf{s}\left(t_{r}, t_{a}, \Theta\right)\right]^{\dagger} \mathbf{\Sigma}_{\mathbf{E}}(\Theta)^{-1}\left[\mathbf{Z}+\mathbf{s}\left(t_{r}, t_{a}, \Theta\right)\right]}
$$

where $\mathbf{s}\left(t_{r}, t_{a}, \Theta\right)$ is the deterministic model of the target. Note that all the signal models for EDPCA are in range time $\left(t_{r}\right)$ and azimuth time $\left(t_{a}\right)$ domain. This is one of the defining characteristic of EDPCA that makes it different from ISTAP, which will be discussed in Section 2.3.4. Using the PDFs of equations 2.68 and 2.70, the EDPCA test statistic is derived in 35 and given as

$$
T_{E D P C A}=\frac{\left|\mathbf{d}_{E}^{\dagger}(\Theta) \mathbf{\Sigma}_{\mathbf{E}}^{-\mathbf{1}}(\Theta) \mathbf{Z}\left(t_{r}, t_{a}, \Theta\right)\right|^{2}}{\mathbf{d}_{E}^{\dagger}(\Theta) \boldsymbol{\Sigma}_{\mathbf{E}}^{-\mathbf{1}}(\Theta) \mathbf{d}_{E}(\Theta)}
$$


where $\mathbf{d}_{E}(\Theta)$ is called the "steering vector", and it compensates the phase difference between the channels. It is a function of the moving target Doppler shift $\left(\frac{2 V_{\mathrm{rt}}}{\lambda}\right)$ and the baseline delay $\left(t_{b}\right)$ between the channels

$$
\mathbf{d}_{E}(\Theta)=\left[\begin{array}{c}
\mathrm{e}^{-j 2 \pi \frac{2 V_{\mathrm{rt}}^{\perp}}{\lambda} t_{b, 1}(\Theta)} \\
\mathrm{e}^{-j 2 \pi \frac{2 V_{\mathrm{rt}}^{\frac{1}{\lambda}}}{\lambda} t_{b, 1}(\Theta)} \\
\vdots \\
\mathrm{e}^{-j 2 \pi \frac{2 V_{\mathrm{rt}}^{\perp}}{\lambda} t_{b, M}(\Theta)}
\end{array}\right] .
$$

The operation of Equation 2.71 can be understood by first examining the quadratic form in the numerator. The multi-channel data $\mathbf{Z}\left(t_{r}, t_{a}, \Theta\right)$ is the time-domain received signal that has been range and azimuth compressed with the target parameters $\Theta$. This maximizes the SNR of the target in each received signal. Multiplication of the signal vector with the inverse convariance matrix performs clutter cancellation. Note that for a two-channel signal vector, the clutter cancellation product reduces to a form that is similar to Equation 2.65 for conventional DPCA 33]. The multiplication of this product with the steering vector compensates for the phase difference between the channels and coherently sums all the contributions of the moving target in the different receive channels [35. The term in the denominator of Equation 2.71 normalizes the test statistic. After normalization, detection is performed in the image domain by comparing the value of each $\left(t_{r}, t_{a}\right)$ pixel with the CFAR threshold, as shown in Equation 2.67.

The test statistic is computed and thresholded iteratively for different target parameters $(\Theta)$. Parameter estimation is then performed by amalgamating all the detections from all the test statistics 35.

\subsubsection{Imaging Space-Time Adaptive Processing}

Imaging Space-Time Adaptive Processing (ISTAP) is an extension of STAP to spaceborne SAR, In literature, STAP is an umbrella term for algorithms that perform spatial and temporal filtering on signals from adaptive array sensors $10-12$. A pulse-doppler radar built using multiple antenna elements is an example of array of spatially distributed sensors, which processes multiple temporal snapshots. Radars employing STAP techniques are typically used to detect and locate moving targets in environments with severe interference, which includes clutter, noise, and jamming.

Robust filtering in spatial and temporal domains requires suitable models for moving 
targets, clutter, and jammers [10,12]. A radar system using STAP for target detection and estimation would use the same hypothesis and GLRT discussed in equations 2.66a 2.67. Generally, models employing Gaussian interference statistics for STAP form a test-statistic of the following form $10,12,37$

$$
T_{S T A P}=\frac{\left|\mathbf{d}_{S}^{\dagger}(\Theta) \mathbf{\Sigma}_{\mathbf{S}}^{-1} \mathbf{Z}_{\mathbf{S}}\right|^{2}}{\mathbf{d}_{S}^{\dagger}(\Theta) \Sigma_{\mathbf{S}}^{-1}(\Theta) \mathbf{d}_{S}(\Theta)}
$$

where $\mathbf{d}_{S}(\Theta)$ is the steering vector, $\boldsymbol{\Sigma}_{\mathbf{S}}^{-\mathbf{1}}$ is the inverse of the interference model covariance matrix, $\mathbf{Z}_{\mathbf{S}}$ is the spatial-temporal signal from the array sensor, and $\Theta$ is the parameter set of the target model. The exact structure of these functions depends on the specific models that are used. Note the resemblance of the EDPCA test statistic in Equation 2.71 to Equation 2.73. In fact, DPCA and EDPCA are just specific cases of STAP 10,11,35.

ISTAP is a combination of STAP and space-borne SAR. STAP based algorithms for airborne radar, for example "post-Doppler STAP", perform clutter cancellation in Doppler domain over small "coherent processing interval" (CPI) segments of the data [11, 12]. This means that for target detection, these methods only employ a few samples from the interference model. This leads to a reduction in signal SNR, which causes a significant problem for space-borne radars since the SNR is already so low [4]. ISTAP tries to mitigate this problem by performing clutter cancellation in the doppler domain, however, instead of using the small CPIs, the entire data is coherently processed [1, 4]. This is a defining characteristic of ISTAP, as it ensures that all the contributions of the target are included in the processing. The ISTAP test statistic is as follows

$$
T_{I S T A P}\left(t_{r}, t_{a}, \Theta\right)=\frac{\left|\int h\left(f_{d}, \Theta\right) \mathbf{d}_{I}^{\dagger}\left(f_{d}, \Theta\right) \mathbf{\Sigma}_{\mathbf{I}}^{-\mathbf{1}}\left(f_{d}\right) \mathbf{Z}\left(t_{r}, f_{d}\right) \mathrm{e}^{j 2 \pi f_{d} t_{a}} \mathrm{~d} f_{d}\right|^{2}}{\int \mathbf{d}_{I}^{\dagger}\left(f_{d}, \Theta\right) \boldsymbol{\Sigma}_{\mathbf{I}}^{-\mathbf{1}}\left(f_{d}\right) \mathbf{d}_{I}\left(f_{d}, \Theta\right) \mathrm{d} f_{d}}
$$

where the steering vector $\mathbf{d}_{I}\left(f_{d}, \Theta\right)$, the inverse covariance matrix $\boldsymbol{\Sigma}_{\mathbf{I}}^{-\mathbf{1}}\left(f_{d}\right)$, and the signal vector $\mathbf{Z}\left(t_{r}, f_{d}, \Theta\right)$, are all in range-Doppler (time-frequency) domain. The ISTAP covariance matrix is defined as follows

$$
\boldsymbol{\Sigma}_{\mathbf{I}}=\frac{1}{N_{r}} \sum_{r} \mathbf{Z}\left(t_{r}, f_{d}\right) \mathbf{Z}^{\dagger}\left(t_{r}, f_{d}\right)
$$

The Doppler frequency dependent clutter-plus-noise covariance matrix is estimated by averaging over range cells. The dimensionality of the ISTAP covariance matrix is $M \times$ $M \times N_{a}$, where as the dimensionality of the EDPCA covariance matrix of Equation 2.69 is $M \times M$. This increase in dimensionality potentially provides better clutter cancellation 
over EDPCA. The increase computation due to the higher dimensionality is compensated by the fact that the clutter cancellation has to be performed only once [4]. Furthermore, the ISTAP covariance matrix has a block-diagonal structure, which can be implemented more efficiently than matrices of similar dimensions that don't have a similar structure.

The ISTAP steering vector, that compensates for the phase difference between the different channels is given by [4]

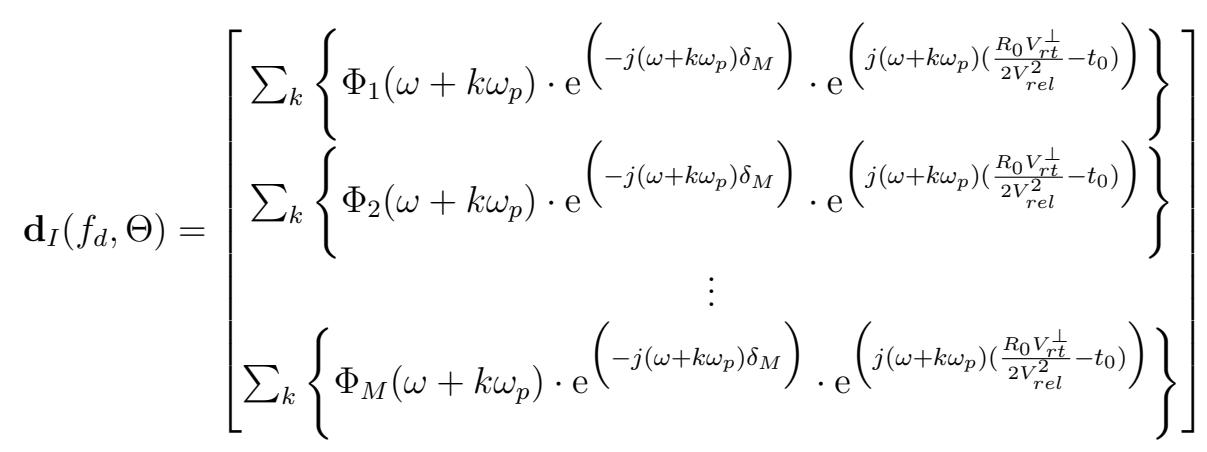

where $\Phi_{M}\left(\omega+k \omega_{p}\right)$ is the two-way antenna pattern for the $M$ th channel

$$
\Phi_{M}\left(\omega+k \omega_{p}\right)=\left(\mathbf{w}_{t, M}^{\dagger} \mathbf{d}\left(u\left(\omega+k \omega_{p}\right)\right)\right) \cdot\left(\mathbf{w}_{r, M}^{\dagger} \mathbf{d}\left(u\left(\omega+k \omega_{p}\right)\right)\right),
$$

the first exponential is due to the frequency shift of the moving target, and the second exponential is due to phase shift of the baseline delay and the sampling delay.

In the test-statistic of Equation 2.74, the integral of the function $h\left(f_{d}, \Theta\right)$ in the numerator represents the SAR compression function that converts the data back in the image (time) domain. The exact form of $h\left(f_{d}, \Theta\right)$ depends on the algorithm used to perform the transformation (RDA, CSA, and etc). After SAR compression, the energy of the target, which is distributed over several range-azimuth cells, is focused at the cells that represent the target. This effectively maximizes the SNR of the moving target with parameter set $\Theta$. The denominator of Equation 2.74normalizes the ISTAP test statistic.

The STAP integrated signal-to-noise-plus-clutter (or signal-to-interference) ratio (SCNR) is another important term that evaluates the SMTI performance.The SCNR term for ISTAP, which is a function of the target radial speed and Doppler frequency, is derived in 13 and expressed as

$$
\operatorname{SCNR}\left(V_{\mathrm{rt}}^{\perp}, f_{d}\right)=\left(\sigma_{s} \exp \left(j \Delta_{s}\right)\right)^{2} \mathbf{d}_{I}^{\dagger}\left(f_{d}, \Theta\right) \boldsymbol{\Sigma}_{\mathbf{I}}^{-1} \mathbf{d}_{I}\left(f_{d}, \Theta\right)
$$




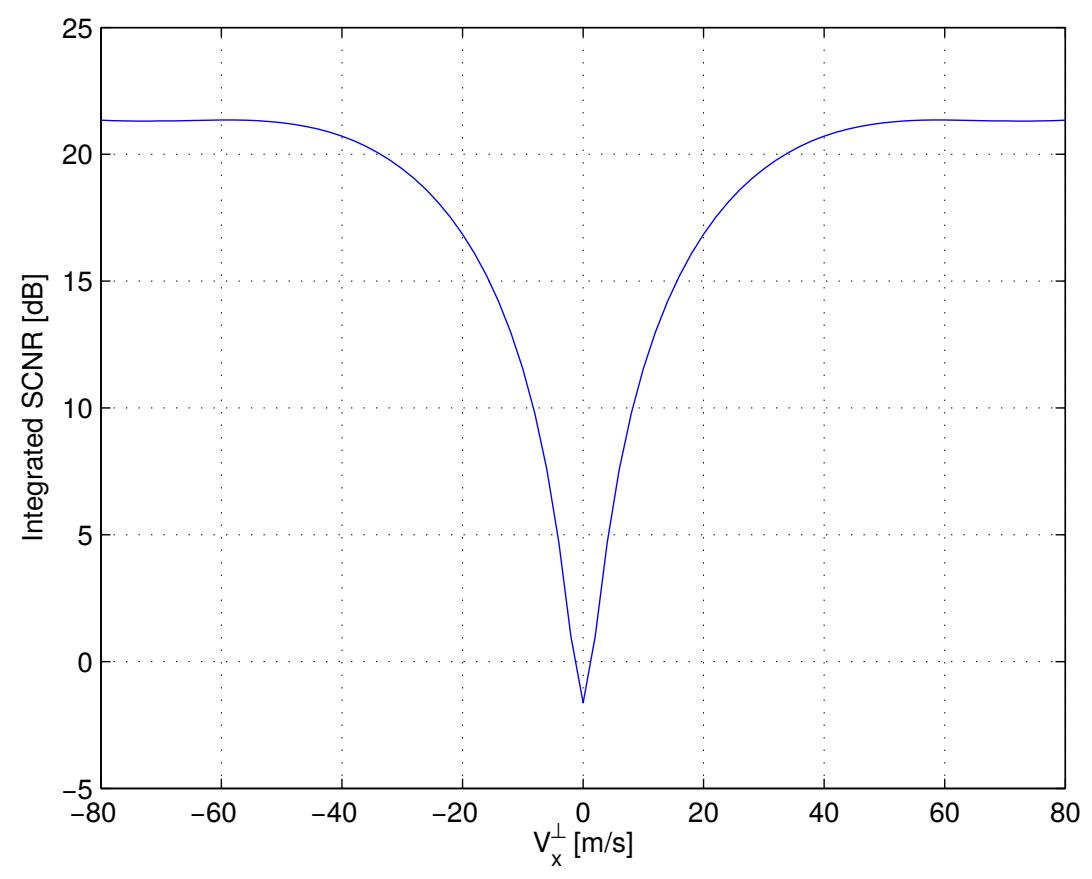

Figure 2.4: ISTAP clutter filter.

This SCNR term is an expression for the STAP clutter filter, which describes the clutter suppression performance. A plot of the SCNR term of Equation 2.78 depends on the system configuration, but is typically of the form shown in Figure 2.4. As expected, the filter is high-pass in nature, with a null at zero (stationary clutter) frequency that suppresses the clutter energy. 


\section{Chapter 3}

\section{Cramér-Rao Lower Bound Derivation}

The CRLB provides a lower bound on the achievable variance of any unbiased estimator. An estimator that achieves this bound is called efficient, however, there is no guarantee that an efficient estimator can be found. Nonetheless, the variance of the efficient estimator provides a good estimate of the capability of the system and serves as a valuable system performance validation tool. Even if an efficient estimator cannot be found, for radar systems the CRLB provides a necessary, but not sufficient design baseline for measurement parameters such as the mode, power levels, pulse-repetition frequency (PRF), platform orbit and attitude, and others. In this chapter, the derivation of the CRLB for the ISTAP signal model of Equation 2.49 in clutter and noise is presented. The CRLB derivation and validation serves as a primary contribution of this thesis.

The signal model that includes the statistical model of clutter can be written as

$$
\mathbf{Z}=\mathbf{s}(\Theta)+\mathbf{W}
$$

where $\mathbf{W}$ represents the clutter-plus-noise model and $\mathbf{s}(\Theta)$ is the moving target signal model of Equation 2.49, which is a function of the following unknown parameter set

$$
\boldsymbol{\Theta}=\left[\begin{array}{c}
\sigma_{s} \\
\Delta_{s} \\
t_{0} \\
V_{\mathrm{x}}^{\perp} \\
V_{\mathrm{x}}
\end{array}\right]
$$


where $\sigma_{s}$ is the unknown target amplitude, $\Delta_{s}$ the unknown target phase, $t_{0}$ is the time at which the target appears in the antenna beam, $V_{\mathrm{x}}$ is the target along-track velocity, and $V_{\mathrm{x}}^{\perp}$ is the target across-track velocity.

The statistical model of clutter plus noise, which is given in Section 3.1, is a multivariate Gaussian PDF. As proved in [13], for a signal model of Equation 3.1, where W is a multivariate Gaussian PDF, the $(m, n)^{t h}$ element of the Fisher information matrix can be written as

$$
[\mathbf{J}(\boldsymbol{\Theta})]_{m, n}=2 * \operatorname{Re}\left\{\frac{\partial \mathbf{s}^{\dagger}(\boldsymbol{\Theta})}{\partial \theta_{m}} \boldsymbol{\Sigma}_{\mathbf{W}}^{-\mathbf{1}} \frac{\partial \mathbf{s}(\boldsymbol{\Theta})}{\partial \theta_{n}}\right\}
$$

where $\mathbf{s}(\boldsymbol{\Theta})$ is the target signal, $\theta_{m}$ and $\theta_{n}$ are the $m^{\text {th }}$ and $n^{\text {th }}$ parameters from Equation 3.2 , and $\Sigma_{\mathbf{W}}^{-1}$ is the inverse of the covariance matrix of the statistical model of clutter. The parameter set in Equation 3.2 yields a $5 \times 5$ Fisher information matrix. The CRLB is given by the inverse of the Fisher information matrix

$$
\operatorname{Cov}(\hat{\Theta}) \geq \mathbf{J}(\Theta)^{-1}
$$

where the variance of any unbiased estimator $\hat{\Theta}$ of the parameter set $\Theta$ is bounded by the inverse of the Fisher information matrix $\mathbf{J}(\Theta)^{-1}$.

\subsection{Statistical model of clutter and noise}

The statistical model of clutter-plus-noise is given as a zero mean complex Gaussian interference

$$
\mathbf{W}=\mathbf{C}+\mathbf{N}
$$

where $\mathbf{N}$ is the additive white Gaussian noise and $\mathbf{C}$ is the clutter model.

The clutter plus noise at each Doppler frequency bin is modeled as a zero mean complex Gaussian signal. Furthermore, the clutter plus noise is assumed to be statistically independent between frequency bins. This model is expressed in Equation 3.6 as

$$
\begin{aligned}
& f_{\overrightarrow{\mathrm{w}}\left(\omega_{1}\right), \overrightarrow{\mathrm{w}}\left(\omega_{2}\right), \ldots, \overrightarrow{\mathrm{w}}\left(\omega_{M}\right)}\left[\overrightarrow{\mathrm{w}}\left(\omega_{1}\right), \overrightarrow{\mathrm{w}}\left(\omega_{2}\right), \ldots, \overrightarrow{\mathrm{w}}\left(\omega_{M}\right)\right] \\
& =\frac{1}{\pi^{M}} \exp \left[\sum_{k=1}^{M}-\overrightarrow{\mathrm{w}}\left(\omega_{k}\right)^{\dagger} \boldsymbol{\Sigma}_{\mathbf{W}}^{-\mathbf{1}}\left(\omega_{k}\right) \overrightarrow{\mathrm{w}}\left(\omega_{k}\right)\right] \prod_{k=1}^{M}\left|\boldsymbol{\Sigma}_{\mathbf{W}}\left(\omega_{k}\right)\right|^{-1}
\end{aligned}
$$

where $\overrightarrow{\mathrm{w}}\left(\omega_{M}\right)$ is the clutter-plus-noise vector at the $\omega_{M}$ frequency bin, $\boldsymbol{\Sigma}_{\mathbf{W}}=E\left[\overrightarrow{\mathrm{w}}(\omega) \overrightarrow{\mathrm{w}}(\omega)^{\dagger}\right]$ is the clutter-plus-noise model covariance matrix and $\boldsymbol{\Sigma}_{\mathbf{W}}^{-1}$ is its inverse. The expression 
for the covariance matrix at each frequency bin is derived in [13] and given as

$$
\begin{aligned}
\mathbf{\Sigma}_{\mathbf{W}}=\sigma_{n}^{2} \mathbf{I}_{M}+\sigma_{c}^{2} \sum_{k}\left\{\left[\left(\mathbf{w}_{t, M}^{\dagger} \mathbf{d}\left(u\left(\omega+k \omega_{p}\right)\right)\right) \cdot\left(\mathbf{w}_{r, M}^{\dagger} \mathbf{d}\left(u\left(\omega+k \omega_{p}\right)\right)\right)\right]^{\dagger}\right. \\
\left.\cdot\left[\left(\mathbf{w}_{t, M}^{\dagger} \mathbf{d}\left(u\left(\omega+k \omega_{p}\right)\right)\right) \cdot\left(\mathbf{w}_{r, M}^{\dagger} \mathbf{d}\left(u\left(\omega+k \omega_{p}\right)\right)\right)\right]\right\} .
\end{aligned}
$$

\subsection{Partial Derivatives}

The derivation of CRB requires partial derivatives of the signal as shown in Equation 3.3 with respect to the target parameters of Equation 3.2. These derivatives are presented in this section. Additionally, a validation of the derivatives is also performed for parameters that yield a non-trivial expression. All the derivations in the following subsections are the primary contributions of this thesis.

For readability, the signal model of Equation 2.49, which is used to derive all the partial derivatives, is duplicated in Equation 3.9 below.

$$
\begin{array}{r}
\mathbf{s}(\Theta)=\sigma_{s} \exp \left(j \Delta_{s}\right)\left(\sqrt { \frac { R _ { 0 } \lambda } { j 2 V _ { r e l } ^ { 2 } } } \sum _ { k } \left\{\left(\mathbf{w}_{t, M}^{\dagger} \mathbf{d}\left(u\left(\omega+k \omega_{p}\right)\right)\right) \cdot\left(\mathbf{w}_{r, M}^{\dagger} \mathbf{d}\left(u\left(\omega+k \omega_{p}\right)\right)\right)\right.\right. \\
\exp \left(-j\left(\omega+k \omega_{p}\right) \delta_{M}\right) \exp \left(j\left(\omega+k \omega_{p}\right)\left(\frac{R_{0} V_{r t}^{\perp}}{2 V_{r e l}^{2}}-t_{0}\right)\right) \\
\left.\left.\exp \left(-2 j \beta R_{0} \sqrt{1-\left(\frac{V_{r t}^{\perp}}{V_{r e l}}\right)^{2}} \sqrt{1-\frac{\left(\omega+k \omega_{p}\right)^{2}}{4 \beta^{2} V_{r e l}^{2}}}\right)\right\}\right)
\end{array}
$$

\subsubsection{Partial derivative of $\mathrm{s}(\Theta)$ with respect to $\sigma_{s}$}

The partial derivative of the signal model with respect to the unknown target amplitude $\sigma_{s}$ was derived and given below. This derivation, though straightforward, is one of the contributions of this thesis. 


$$
\begin{array}{r}
\frac{\partial \mathbf{s}(\boldsymbol{\Theta})}{\partial \sigma_{s}}=\exp \left(j \Delta_{s}\right)\left(\sqrt { \frac { R _ { 0 } \lambda } { j 2 V _ { r e l } ^ { 2 } } } \sum _ { k } \left\{\left(\mathbf{w}_{t, M}^{\dagger} \mathbf{d}\left(u\left(\omega+k \omega_{p}\right)\right)\right) \cdot\left(\mathbf{w}_{r, M}^{\dagger} \mathbf{d}\left(u\left(\omega+k \omega_{p}\right)\right)\right)\right.\right. \\
\exp \left(-j\left(\omega+k \omega_{p}\right) \delta_{M}\right) \exp \left(j\left(\omega+k \omega_{p}\right)\left(\frac{R_{0} V_{r t}^{\perp}}{2 V_{r e l}^{2}}-t_{0}\right)\right) \\
\left.\left.\exp \left(-2 j \beta R_{0} \sqrt{1-\left(\frac{V_{r t}^{\perp}}{V_{r e l}}\right)^{2}} \sqrt{1-\frac{\left(\omega+k \omega_{p}\right)^{2}}{4 \beta^{2} V_{r e l}^{2}}}\right)\right\}\right) .
\end{array}
$$

\subsubsection{Partial derivative of $\mathrm{s}(\Theta)$ with respect to $\Delta_{s}$}

The partial derivative of the signal model with respect to the unknown target phase $\Delta_{s}$ was derived and is given in Equation 3.11 below. This derivation is also one of the contributions of this thesis.

$$
\begin{array}{r}
\frac{\partial \mathbf{s}(\boldsymbol{\Theta})}{\partial \Delta_{s}}=j \sigma_{s} \exp \left(j \Delta_{s}\right)\left(\sqrt { \frac { R _ { 0 } \lambda } { j 2 V _ { r e l } ^ { 2 } } } \sum _ { k } \left\{\left(\mathbf{w}_{t, M}^{\dagger} \mathbf{d}\left(u\left(\omega+k \omega_{p}\right)\right)\right) \cdot\left(\mathbf{w}_{r, M}^{\dagger} \mathbf{d}\left(u\left(\omega+k \omega_{p}\right)\right)\right)\right.\right. \\
\exp \left(-j\left(\omega+k \omega_{p}\right) \delta_{M}\right) \exp \left(j\left(\omega+k \omega_{p}\right)\left(\frac{R_{0} V_{r t}^{\perp}}{2 V_{r e l}^{2}}-t_{0}\right)\right) \\
\left.\left.\exp \left(-2 j \beta R_{0} \sqrt{1-\left(\frac{V_{r t}^{\perp}}{V_{r e l}}\right)^{2}} \sqrt{1-\frac{\left(\omega+k \omega_{p}\right)^{2}}{4 \beta^{2} V_{r e l}^{2}}}\right)\right\}\right) .
\end{array}
$$

\subsubsection{Partial derivative of $\mathrm{s}(\Theta)$ with respect to $t_{0}$}

The partial derivative of the signal model with respect to the target crossing time $t_{0}$ was derived and is given in Equation 3.12 below. This derivation is also one of the contributions of this thesis. 


$$
\begin{aligned}
& \frac{\partial \mathbf{s}(\boldsymbol{\Theta})}{\partial t_{0}}=\sigma_{s} \exp \left(j \Delta_{s}\right) \sqrt{\frac{R_{0} \lambda}{j 2 V_{r e l}^{2}}} \sum_{k}\left\{\left(\mathbf{w}_{t, M}^{\dagger} \mathbf{d}\left(u\left(\omega+k \omega_{p}\right)\right)\right) \cdot\left(\mathbf{w}_{r, M}^{\dagger} \mathbf{d}\left(u\left(\omega+k \omega_{p}\right)\right)\right)\right. \\
& \exp \left(-j\left(\omega+k \omega_{p}\right) \delta_{M}\right) \\
& \exp \left(j\left(\omega+k \omega_{p}\right)\left(\frac{R_{0} V_{r t}^{\perp}}{2 V_{r e l}^{2}}\right)\right) \\
& \exp \left(-2 j \beta R_{0} \sqrt{1-\left(\frac{V_{r t}^{\perp}}{V_{r e l}}\right)^{2}} \sqrt{1-\frac{\left(\omega+k \omega_{p}\right)^{2}}{4 \beta^{2} V_{r e l}^{2}}}\right) \\
& \left.\left(-j\left(\omega+k \omega_{p}\right)\right) \exp \left(-j\left(\omega+k \omega_{p}\right)\left(t_{0}\right)\right)\right\} \text {. }
\end{aligned}
$$

\subsubsection{Partial derivative of $\mathrm{s}(\Theta)$ with respect to $V_{\mathrm{x}}$}

The derivative of the signal with respect to the parameter $V_{\mathrm{x}}$ yields a non-trivial expression. Therefore, in order for the derivation to be more tractable, the expression for the signal in Equation 3.9 is divided into smaller factors, as shown in equations 3.133 .16 . This derivation is also one of the primary contributions of this thesis.

$$
\begin{gathered}
\mathrm{H}\left(V_{\mathrm{x}} ; \boldsymbol{\Theta}^{c}\right)=\sqrt{\frac{R_{0} \lambda}{j 2 V_{r e l}^{2}}} \\
\mathrm{I}_{\mathrm{k}}\left(V_{\mathrm{x}} ; \boldsymbol{\Theta}^{c}\right)=\left(\mathbf{w}_{t, M}^{\dagger} \mathbf{d}\left(u\left(\omega+k \omega_{p}\right)\right)\right) \cdot\left(\mathbf{w}_{r, M}^{\dagger} \mathbf{d}\left(u\left(\omega+k \omega_{p}\right)\right)\right) \exp \left(-j\left(\omega+k \omega_{p}\right)\left(\delta_{M}+t_{0}\right)\right) \\
\mathrm{M}_{\mathrm{k}}\left(V_{\mathrm{x}} ; \Theta^{c}\right)=\exp \left(j\left(\omega+k \omega_{p}\right)\left(\frac{R_{0} V_{r t}^{\perp}}{2 V_{r e l}^{2}}\right)\right) \\
\mathrm{N}_{\mathrm{k}}\left(V_{\mathrm{x}} ; \boldsymbol{\Theta}^{c}\right)=\exp \left(-2 j \beta R_{0} \sqrt{1-\left(\frac{V_{r t}^{\perp}}{V_{r e l}}\right)^{2}} \sqrt{1-\frac{\left(\omega+k \omega_{p}\right)^{2}}{4 \beta^{2} V_{r e l}^{2}}}\right)
\end{gathered}
$$

Using these factors, the signal model $\mathbf{s}(\boldsymbol{\Theta})$ can be written as

$$
\mathbf{s}(\boldsymbol{\Theta})=\sigma_{s} \exp \left(j \Delta_{s}\right) \mathrm{H}\left(V_{\mathrm{x}} ; \boldsymbol{\Theta}^{c}\right) \sum_{k}\left\{\mathrm{I}_{\mathrm{k}}\left(V_{\mathrm{x}} ; \boldsymbol{\Theta}^{c}\right) \mathrm{M}_{\mathrm{k}}\left(V_{\mathrm{x}} ; \boldsymbol{\Theta}^{c}\right) \mathrm{N}_{\mathrm{k}}\left(V_{\mathrm{x}} ; \boldsymbol{\Theta}^{c}\right)\right\}
$$


and a simpler form of the derivative is obtained by using the chain-rule, which is given as follow 1

$$
\begin{aligned}
\frac{\partial \mathbf{s}(\boldsymbol{\Theta})}{\partial V_{\mathrm{x}}} & =\sigma_{s} \exp \left(j \Delta_{s}\right) \frac{\partial \mathrm{H}\left(V_{\mathrm{x}} ; \boldsymbol{\Theta}^{c}\right)}{\partial V_{\mathrm{x}}} \sum_{k}\left\{\mathrm{I}_{\mathrm{k}}\left(V_{\mathrm{x}} ; \boldsymbol{\Theta}^{c}\right) \mathrm{M}_{\mathrm{k}}\left(V_{\mathrm{x}} ; \boldsymbol{\Theta}^{c}\right) \mathrm{N}_{\mathrm{k}}\left(V_{\mathrm{x}} ; \boldsymbol{\Theta}^{c}\right)\right\} \\
& +\sigma_{s} \exp \left(j \Delta_{s}\right) \mathrm{H}\left(V_{\mathrm{x}} ; \boldsymbol{\Theta}^{c}\right) \sum_{k}\left\{\frac{\partial \mathrm{I}_{\mathrm{k}}\left(V_{\mathrm{x}} ; \boldsymbol{\Theta}^{c}\right)}{\partial V_{\mathrm{x}}} \mathrm{M}_{\mathrm{k}}\left(V_{\mathrm{x}} ; \boldsymbol{\Theta}^{c}\right) \mathrm{N}_{\mathrm{k}}\left(V_{\mathrm{x}} ; \boldsymbol{\Theta}^{c}\right)\right\} \\
& +\sigma_{s} \exp \left(j \Delta_{s}\right) \mathrm{H}\left(V_{\mathrm{x}} ; \boldsymbol{\Theta}^{c}\right) \sum_{k}\left\{\mathrm{I}_{\mathrm{k}}\left(V_{\mathrm{x}} ; \boldsymbol{\Theta}^{c}\right) \frac{\partial \mathrm{M}_{\mathrm{k}}\left(V_{\mathrm{x}} ; \boldsymbol{\Theta}^{c}\right)}{\partial V_{\mathrm{x}}} \mathrm{N}_{\mathrm{k}}\left(V_{\mathrm{x}} ; \boldsymbol{\Theta}^{c}\right)\right\} \\
& +\sigma_{s} \exp \left(j \Delta_{s}\right) \mathrm{H}\left(V_{\mathrm{x}} ; \boldsymbol{\Theta}^{c}\right) \sum_{k}\left\{\mathrm{I}_{\mathrm{k}}\left(V_{\mathrm{x}} ; \boldsymbol{\Theta}^{c}\right) \mathrm{M}_{\mathrm{k}}\left(V_{\mathrm{x}} ; \boldsymbol{\Theta}^{c}\right) \frac{\partial \mathrm{N}_{\mathrm{k}}\left(V_{\mathrm{x}} ; \boldsymbol{\Theta}^{c}\right)}{\partial V_{\mathrm{x}}}\right\} .
\end{aligned}
$$

The partial derivative $\frac{\partial \mathrm{H}\left(V_{\mathrm{x}} ; \boldsymbol{\Theta}^{c}\right)}{\partial V_{\mathrm{x}}}$ is derived as

$$
\begin{aligned}
\frac{\partial \mathrm{H}\left(V_{\mathrm{x}} ; \boldsymbol{\Theta}^{c}\right)}{\partial V_{\mathrm{x}}}= & \frac{-\sqrt{R_{0} \lambda}\left(\frac{1}{2}\right)\left(j 2 V_{r e l}^{2}\right)^{-\frac{1}{2}} 4 j V_{r e l} \frac{\left(V_{x}-V_{a}\right)}{V_{r e l}}}{j 2 V_{r e l}^{2}} \\
& =\frac{-(2 j) \sqrt{R_{0} \lambda}\left(V_{x}-V_{a}\right)}{\left(\sqrt{j 2 V_{r e l}^{2}}\right)^{3}}
\end{aligned}
$$

where $\frac{\partial V_{r e l}}{\partial V_{\mathrm{x}}}=\frac{V_{\mathrm{x}}-V_{a}}{V_{r e l}}$. The partial derivative $\frac{\partial \mathrm{I}_{\mathrm{k}}\left(V_{\mathrm{x}} ; \boldsymbol{\Theta}^{c}\right)}{\partial V_{\mathrm{x}}}$ is computed as

$$
\begin{aligned}
\frac{\partial \mathrm{I}_{\mathrm{k}}\left(V_{\mathrm{x}} ; \boldsymbol{\Theta}^{c}\right)}{\partial V_{\mathrm{x}}} & =\frac{\partial\left\{\left(\mathbf{w}_{t, M}^{\dagger} \mathbf{d}\left(u\left(\omega+k \omega_{p}\right)\right)\right) \cdot\left(\mathbf{w}_{r, M}^{\dagger} \mathbf{d}\left(u\left(\omega+k \omega_{p}\right)\right)\right)\right\}}{\partial u(w)} \\
& \frac{\partial u(\omega)}{\partial V_{x}} \cdot \exp \left(-j\left(\omega+k \omega_{p}\right)\left(\delta_{M}+t_{0}\right)\right) \\
& =\left\{\mathbf{w}_{t, m}^{\dagger} \frac{\partial \mathbf{d}\left(u\left(\omega+k \omega_{p}\right)\right)}{\partial u(\omega)} \mathbf{w}_{r, M}^{\dagger} \mathbf{d}\left(u\left(\omega+k \omega_{p}\right)\right)+\right. \\
& \left.\mathbf{w}_{t, M}^{\dagger} \mathbf{d}\left(u\left(\omega+k \omega_{p}\right)\right) \mathbf{w}_{r, M}^{\dagger} \frac{\partial \mathbf{d}\left(u\left(\omega+k \omega_{p}\right)\right)}{\partial u(\omega)}\right\} . \\
& \exp \left(-j\left(\omega+k \omega_{p}\right)\left(\delta_{M}+t_{0}\right)\right) \frac{\partial u(\omega)}{\partial V_{x}}
\end{aligned}
$$

${ }^{1}$ Note that $\Theta^{c}$ denotes the target parameter subset that contains all of the parameters from the set Equation 3.2 except $V_{\mathrm{x}}$. 
where the partial derivatives $\frac{\partial \mathbf{d}\left(u\left(\omega+k \omega_{p}\right)\right)}{\partial u(\omega)}$ and $\frac{\partial u(\omega)}{\partial V_{x}}$ are computed as follows

$$
\begin{gathered}
\frac{\partial \mathbf{d}\left(u\left(\omega+k \omega_{p}\right)\right)}{\partial u(\omega)}=\frac{\partial E_{e}(u)}{\partial u}\left[\begin{array}{c}
\exp \left(-j \beta x_{1} u\right) \\
\exp \left(-j \beta x_{2} u\right) \\
\vdots \\
\exp \left(-j \beta x_{N} u\right)
\end{array}\right]+E_{e}(u)\left[\begin{array}{c}
-j \beta x_{1} \exp \left(-j \beta x_{1} u\right) \\
-j \beta x_{2} \exp \left(-j \beta x_{2} u\right) \\
\vdots \\
-j \beta x_{N} \exp \left(-j \beta x_{N} u\right)
\end{array}\right] \\
\frac{\partial u\left(\omega+k \omega_{p}\right)}{\partial V_{x}}=-\left(\left(\omega+k \omega_{p}\right)\right)\left[\frac{V_{\mathrm{rel}}^{2}+2\left(V_{\mathrm{g}}-V_{\mathrm{x}}\right)\left(V_{\mathrm{x}}-V_{\mathrm{a}}\right)}{\left(2 \beta V_{\mathrm{rel}}^{4}\right)} \sqrt{\frac{1-\left(\frac{V_{\mathrm{rt}}^{\perp}}{V_{\mathrm{rel}}}\right)^{2}}{1-\left(\frac{\omega+k \omega_{p}}{2 \beta V_{\mathrm{rel}}}\right)^{2}}}\right. \\
+\frac{\left(\omega+k \omega_{p}\right)\left(V_{\mathrm{g}}-V_{\mathrm{x}}\right)\left(V_{\mathrm{x}}-V_{\mathrm{a}}\right)}{V_{\mathrm{rel}}^{2}} \sqrt{\frac{\left(2 \beta V_{\mathrm{rel}}\right)^{2}-\left(\omega+k \omega_{p}\right)^{2}}{V_{\mathrm{rel}}^{2}-V_{\mathrm{rt}}^{\perp}}\left[\frac{\left(\left(2 \beta V_{\mathrm{rt}}^{\perp}\right)^{2}-\left(\omega+k \omega_{p}\right)^{2}\right)}{\left(\left(2 \beta V_{\mathrm{rel}}\right)^{2}-\left(\omega+k \omega_{p}\right)^{2}\right)^{2}}\right.} \\
-\frac{V_{\mathrm{rt}}^{\perp}\left(V_{\mathrm{rel}}^{2}+2\left(V_{\mathrm{g}}-V_{\mathrm{x}}\right)\left(V_{\mathrm{x}}-V_{\mathrm{a}}\right)\right)}{V_{\mathrm{rel}}^{4}} .
\end{gathered}
$$

The partial derivative $\frac{\partial \mathrm{M}_{\mathrm{k}}\left(V_{\mathrm{x}} ; \boldsymbol{\Theta}^{c}\right)}{\partial V_{\mathrm{x}}}$ is computed as

$$
\begin{aligned}
\frac{\partial \mathrm{M}_{\mathrm{k}}\left(V_{\mathrm{x}} ; \boldsymbol{\Theta}^{c}\right)}{\partial V_{\mathrm{x}}} & =j\left(\omega+k \omega_{p}\right) \frac{-R_{0} V_{r t}^{\perp}\left(4 V_{r e l}\right) \frac{\left(V_{x}-V_{a}\right)}{V_{r e l}}}{\left(2 V_{r e l}^{2}\right)^{2}} \exp \left(j\left(\omega+k \omega_{p}\right)\left(\frac{R_{0} V_{r t}^{\perp}}{2 V_{r e l}^{2}}\right)\right) \\
& =j\left(\omega+k \omega_{p}\right) \frac{-R_{0} V_{r t}^{\perp}\left(4\left(V_{x}-V_{a}\right)\right)}{\left(2 V_{r e l}^{2}\right)^{2}} \exp \left(j\left(\omega+k \omega_{p}\right)\left(\frac{R_{0} V_{r t}^{\perp}}{2 V_{r e l}^{2}}\right)\right) .
\end{aligned}
$$

The partial derivative $\frac{\partial \mathrm{N}_{\mathrm{k}}\left(V_{\mathrm{x}} ; \boldsymbol{\Theta}^{c}\right)}{\partial V_{\mathrm{x}}}$ is computed as

$$
\begin{gathered}
\frac{\partial \mathrm{N}_{\mathrm{k}}\left(V_{\mathrm{x}} ; \boldsymbol{\Theta}^{c}\right)}{\partial V_{\mathrm{x}}}=-2 j \beta R_{0}\left[\frac{V_{\mathrm{rt}}^{\perp}\left(V_{\mathrm{x}}-V_{\mathrm{a}}\right)}{V_{\mathrm{rel}}^{4}} \sqrt{\frac{1-\left(\frac{\left(\omega+k \omega_{p}\right)}{2 \beta V_{\mathrm{rel}}}\right)^{2}}{1-\left(\frac{V_{\mathrm{rt}}^{\perp}}{V_{\mathrm{rel}}}\right)^{2}}+\frac{\left(\omega+k \omega_{p}\right)^{2}\left(V_{\mathrm{x}}-V_{\mathrm{a}}\right)}{\left(2 \beta V_{\mathrm{rel}}^{2}\right)^{2}}}\right. \\
\sqrt{\left.\frac{1-\left(\frac{V_{\mathrm{rt}}^{\perp}}{V_{\mathrm{rel}}}\right)^{2}}{1-\left(\frac{\left(\omega+k \omega_{p}\right)}{2 \beta V_{\mathrm{rel}}}\right)^{2}}\right] \cdot \exp \left(-2 j \beta R_{0} \sqrt{1-\left(\frac{V_{r t}^{\perp}}{V_{\text {rel }}}\right)^{2}} \sqrt{1-\frac{\left(\omega+k \omega_{p}\right)^{2}}{4 \beta^{2} V_{\text {rel }}^{2}}}\right) .}
\end{gathered}
$$




\section{Validation of the Derivative}

When expanded, the number of terms in Equation 3.18 lead to a expression that is non-trivial. Therefore, the derivative needs to be validated in order to discount the possibility of any errors made during the derivation. Validation was performed by using a numerical method to compute the derivative of the signal in Equation 3.17, and comparing the results with the derivative that was derived analytically. Basic methods for numerical differentiation are based on the centered divided-difference formulae or backward divided-difference formulae [38]. However, a naive implementation of these methods yields substantially large round-off and truncation errors. An implementation that tries to minimize these errors is based on Ridders' method of polynomial extrapolation and is provided in Numerical Recipes text [39,40]. This approach was used to compute the numerical derivative.

Figures 3.13 .3 show the signal derivative versus the parameter $V_{\mathrm{x}}$ using the two methods at three different frequencies: $1 \mathrm{rad} / \mathrm{s}, 2 \mathrm{rad} / \mathrm{s}$, and $3 \mathrm{rad} / \mathrm{s}$. The red line shows the derivative that was derived analytically, and the green line shows the derivative that was computed numerically. The figures show that the two derivatives are in agreement, however, the numerical derivative does oscillate around the analytical derivative. The oscillations can be attributed to the fact that the derivative does not change much over the values of $V_{x}$ tested. The algorithm used for the computation of numerical derivative works better when the derivative changes substantially over the independent variable [39.

The error term, which is defined as the difference between the two derivatives is provided in Figure 3.4. The error term is on the order of $10^{-5}$ and oscillates due to the error in the numerical computation. The error term is defined as the absolute value of the difference between the two derivatives and averaged over all the frequencies.

$$
\operatorname{Error} V_{\mathrm{x}}=\left\langle\left|\frac{\partial \mathbf{s}(\boldsymbol{\Theta})}{\partial V_{\mathrm{x}}}-\frac{\partial \mathbf{s}_{n}(\boldsymbol{\Theta})}{\partial V_{\mathrm{x}}}\right|\right\rangle
$$



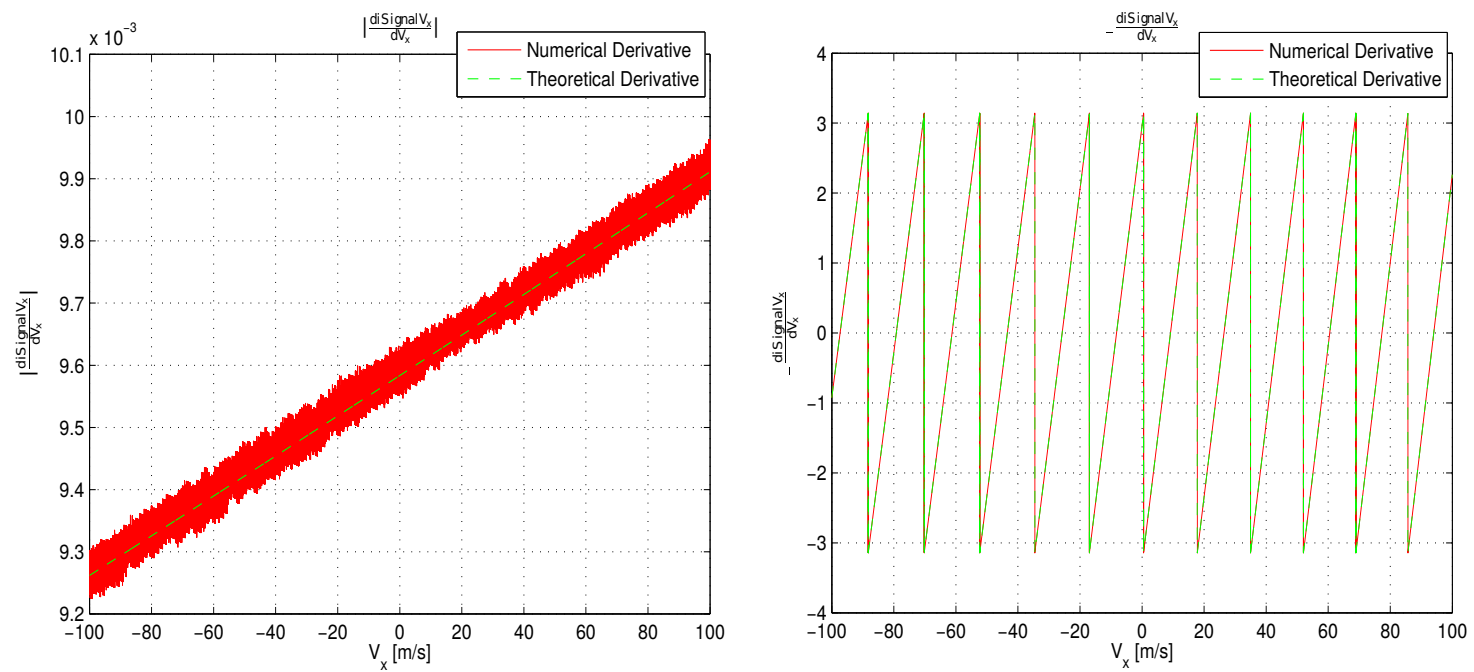

Figure 3.1: Partial derivative with respect to parameter $V_{\mathrm{x}}$ at $1 \mathrm{rad} / \mathrm{s}$.
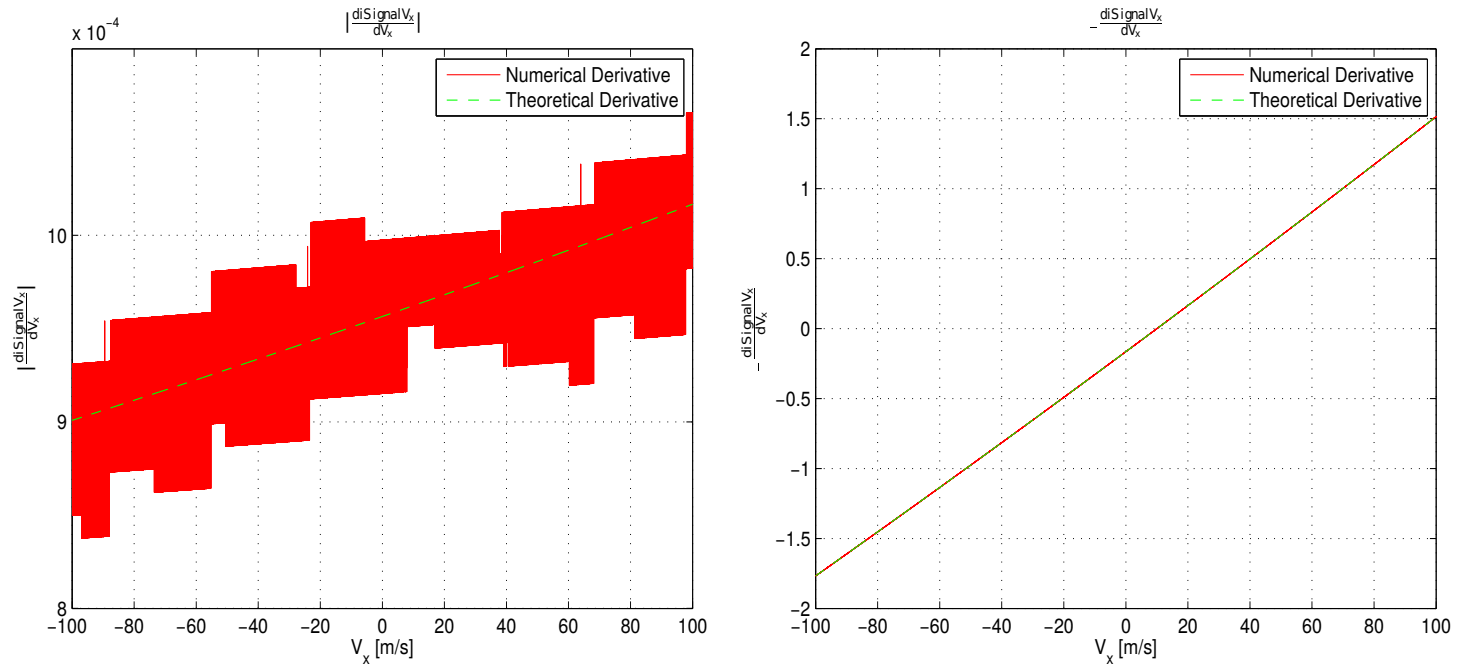

Figure 3.2: Partial derivative with respect to parameter $V_{\mathrm{x}}$ at $2 \mathrm{rad} / \mathrm{s}$. 

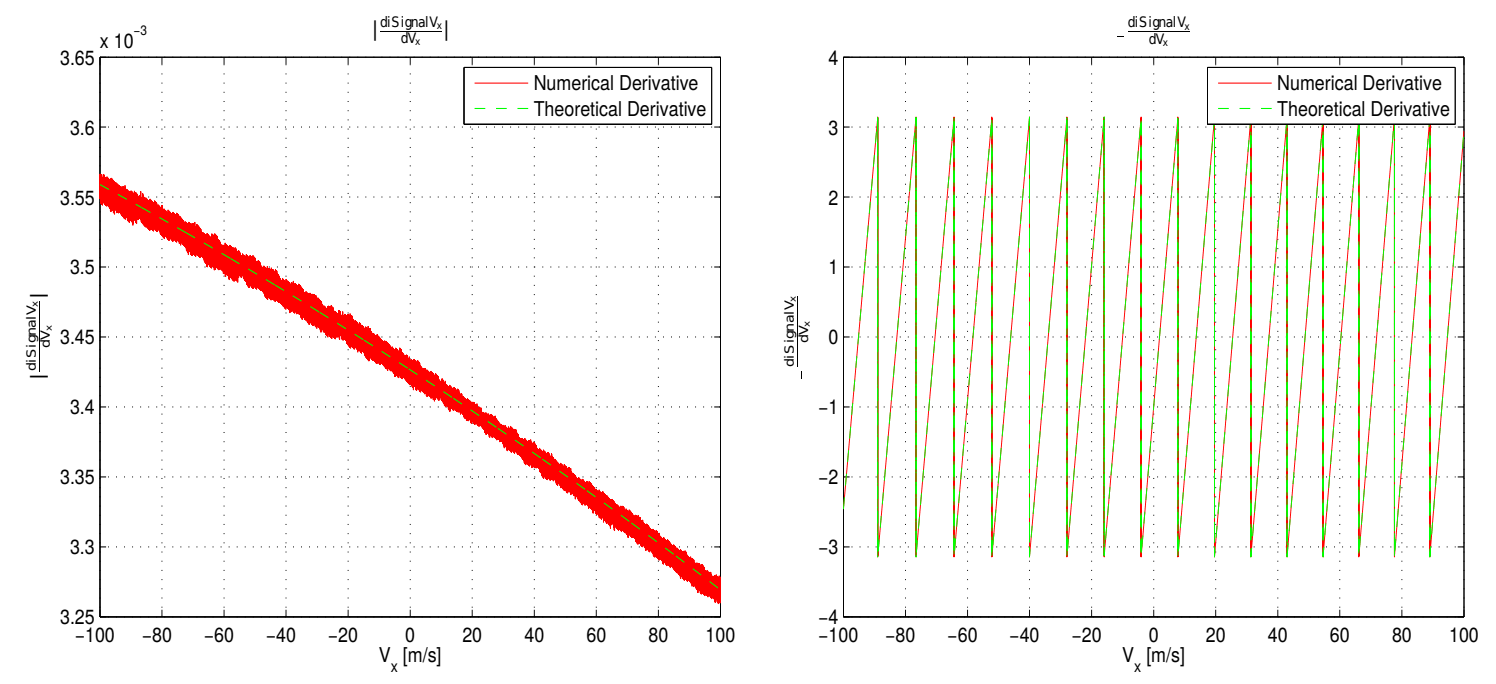

Figure 3.3: Partial derivative with respect to parameter $V_{\mathrm{x}}$ at $3 \mathrm{rad} / \mathrm{s}$.

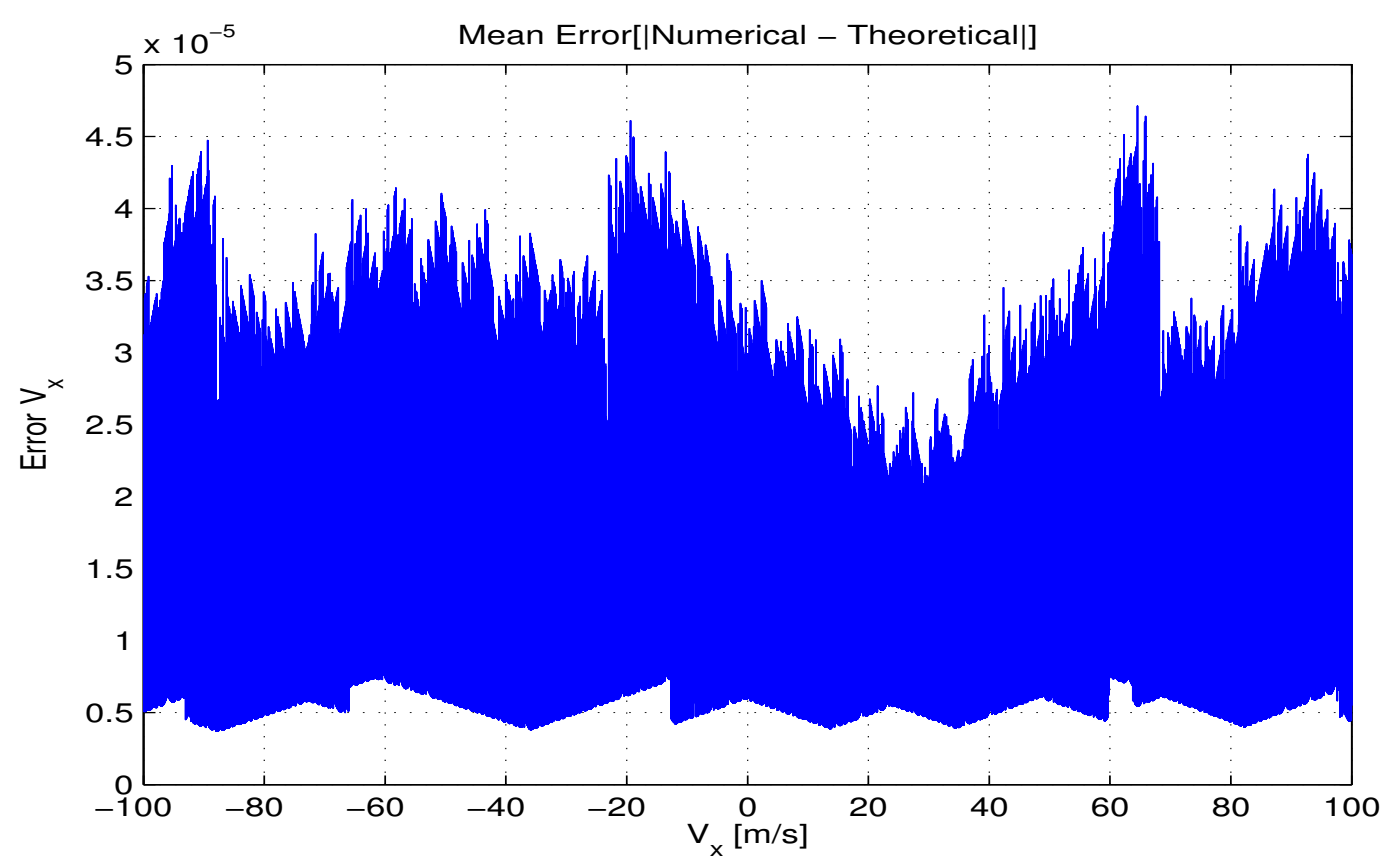

Figure 3.4: Error term for the numerical and theoretical derivative of $V_{\mathrm{x}}$. 


\subsubsection{Partial derivative of $\mathrm{s}(\Theta)$ with respect to $V_{\mathrm{x}}^{\perp}$}

The derivative of the signal with respect to $V_{\mathrm{x}}^{\perp}$ is derived in a similar manner as done for the parameter $V_{\mathrm{x}}$ above. The signal model is divided into smaller factors, as shown in equations 3.263 .29 . This derivation is also one of the primary contributions of this thesis.

$$
\begin{gathered}
\mathrm{H}\left(V_{\mathrm{x}}^{\perp} ; \boldsymbol{\Theta}^{c}\right)=\sqrt{\frac{R_{0} \lambda}{j 2 V_{r e l}^{2}}} \\
\mathrm{I}_{\mathrm{k}}\left(V_{\mathrm{x}}^{\perp} ; \Theta^{c}\right)=\left(\mathbf{w}_{t, M}^{\dagger} \mathbf{d}\left(u\left(\omega+k \omega_{p}\right)\right)\right) \cdot\left(\mathbf{w}_{r, M}^{\dagger} \mathbf{d}\left(u\left(\omega+k \omega_{p}\right)\right)\right) \exp \left(-j\left(\omega+k \omega_{p}\right)\left(\delta_{M}+t_{0}\right)\right) \\
\mathrm{M}_{\mathrm{k}}\left(V_{\mathrm{x}}^{\perp} ; \Theta^{c}\right)=\exp \left(j\left(\omega+k \omega_{p}\right)\left(\frac{R_{0} V_{r t}^{\perp}}{2 V_{r e l}^{2}}\right)\right) \\
\mathrm{N}_{\mathrm{k}}\left(V_{\mathrm{x}}^{\perp} ; \Theta^{c}\right)=\exp \left(-2 j \beta R_{0} \sqrt{1-\left(\frac{V_{r t}^{\perp}}{V_{r e l}}\right)^{2}} \sqrt{1-\frac{\left(\omega+k \omega_{p}\right)^{2}}{4 \beta^{2} V_{r e l}^{2}}}\right)
\end{gathered}
$$

Using these terms, the signal model $\mathbf{s}(\boldsymbol{\Theta})$ can be written as follows

$$
\mathbf{s}(\boldsymbol{\Theta})=\sigma_{s} \exp \left(j \Delta_{s}\right) \mathrm{H}\left(V_{\mathrm{x}}^{\perp} ; \boldsymbol{\Theta}^{c}\right) \sum_{k}\left\{\mathrm{I}_{\mathrm{k}}\left(V_{\mathrm{x}}^{\perp} ; \boldsymbol{\Theta}^{c}\right) \mathrm{M}_{\mathrm{k}}\left(V_{\mathrm{x}}^{\perp} ; \boldsymbol{\Theta}^{c}\right) \mathrm{N}_{\mathrm{k}}\left(V_{\mathrm{x}}^{\perp} ; \boldsymbol{\Theta}^{c}\right)\right\}
$$

and a simpler form of the derivative is obtained using chain-rule, which is given as ${ }^{2}$

$$
\begin{aligned}
\frac{\partial \mathbf{s}(\boldsymbol{\Theta})}{\partial V_{\mathrm{x}}^{\perp}} & =\sigma_{s} \exp \left(j \Delta_{s}\right) \frac{\partial \mathrm{H}\left(V_{\mathrm{x}}^{\perp} ; \boldsymbol{\Theta}^{c}\right)}{\partial V_{\mathrm{x}}^{\perp}} \sum_{k}\left\{\mathrm{I}_{\mathrm{k}}\left(V_{\mathrm{x}}^{\perp} ; \boldsymbol{\Theta}^{c}\right) \mathrm{M}_{\mathrm{k}}\left(V_{\mathrm{x}}^{\perp} ; \boldsymbol{\Theta}^{c}\right) \mathrm{N}_{\mathrm{k}}\left(V_{\mathrm{x}}^{\perp} ; \boldsymbol{\Theta}^{c}\right)\right\} \\
& +\sigma_{s} \exp \left(j \Delta_{s}\right) \mathrm{H}\left(V_{\mathrm{x}}^{\perp} ; \boldsymbol{\Theta}^{c}\right) \sum_{k}\left\{\frac{\partial \mathrm{I}_{\mathrm{k}}\left(V_{\mathrm{x}}^{\perp} ; \boldsymbol{\Theta}^{c}\right)}{\partial V_{\mathrm{x}}^{\perp}} \mathrm{M}_{\mathrm{k}}\left(V_{\mathrm{x}}^{\perp} ; \boldsymbol{\Theta}^{c}\right) \mathrm{N}_{\mathrm{k}}\left(V_{\mathrm{x}}^{\perp} ; \boldsymbol{\Theta}^{c}\right)\right\} \\
& +\sigma_{s} \exp \left(j \Delta_{s}\right) \mathrm{H}\left(V_{\mathrm{x}}^{\perp} ; \boldsymbol{\Theta}^{c}\right) \sum_{k}\left\{\mathrm{I}_{\mathrm{k}}\left(V_{\mathrm{x}}^{\perp} ; \boldsymbol{\Theta}^{c}\right) \frac{\partial \mathrm{M}_{\mathrm{k}}\left(V_{\mathrm{x}}^{\perp} ; \boldsymbol{\Theta}^{c}\right)}{\partial V_{\mathrm{x}}^{\perp}} \mathrm{N}_{\mathrm{k}}\left(V_{\mathrm{x}}^{\perp} ; \boldsymbol{\Theta}^{c}\right)\right\} \\
& +\sigma_{s} \exp \left(j \Delta_{s}\right) \mathrm{H}\left(V_{\mathrm{x}}^{\perp} ; \boldsymbol{\Theta}^{c}\right) \sum_{k}\left\{\mathrm{I}_{\mathrm{k}}\left(V_{\mathrm{x}}^{\perp} ; \boldsymbol{\Theta}^{c}\right) \mathrm{M}_{\mathrm{k}}\left(V_{\mathrm{x}}^{\perp} ; \boldsymbol{\Theta}^{c}\right) \frac{\partial \mathrm{N}_{\mathrm{k}}\left(V_{\mathrm{x}}^{\perp} ; \boldsymbol{\Theta}^{c}\right)}{\partial V_{\mathrm{x}}^{\perp}}\right\} .
\end{aligned}
$$

\footnotetext{
${ }^{2}$ Note that $\Theta^{c}$ denotes the target parameter subset that contains all of the parameters from the set
} Equation 3.2 except $V_{\mathrm{x}}^{\perp}$. 
The partial derivative $\frac{\partial \mathrm{H}\left(V_{\mathbf{x}}^{\perp} ; \boldsymbol{\Theta}^{c}\right)}{\partial V_{\mathbf{x}}^{\perp}}$ is computed as

$$
\begin{aligned}
\frac{\partial \mathrm{H}\left(V_{\mathrm{x}}^{\perp} ; \Theta^{c}\right)}{\partial V_{\mathrm{x}}^{\perp}} & =\frac{1}{2} \sqrt{\frac{j 2 V_{r e l}^{2}}{R_{0} \lambda}} \frac{-R_{0} \lambda\left(j 4 V_{r e l}\right)}{\left(j 2 V_{r e l}^{2}\right)} \frac{\partial V_{r e l}}{\partial V_{\mathrm{x}}^{\perp}} \\
& =\frac{-\left(j 2 V_{\mathrm{x}}^{\perp}\right) \sqrt{R_{0} \lambda}}{\left(\sqrt{j 2 V_{r e l}^{2}}\right)^{3}}
\end{aligned}
$$

where $\frac{\partial V_{\text {rel }}}{\partial V_{\mathrm{x}}^{\perp}}=\frac{V_{\mathrm{x}}^{\perp}}{V_{\text {rel }}}$. The partial derivative $\frac{\partial \mathrm{I}_{\mathrm{k}}\left(V_{\mathrm{x}}^{\perp} ; \mathbf{\Theta}^{c}\right)}{\partial V_{\mathrm{x}}^{\perp}}$ is computed as

$$
\begin{aligned}
\frac{\partial \mathrm{I}_{\mathrm{k}}\left(V_{\mathbf{x}}^{\perp} ; \boldsymbol{\Theta}^{c}\right)}{\partial V_{\mathbf{x}}^{\perp}} & =\frac{\partial\left\{\left(\mathbf{w}_{t, M}^{\dagger} \mathbf{d}\left(u\left(\omega+k \omega_{p}\right)\right)\right) \cdot\left(\mathbf{w}_{r, M}^{\dagger} \mathbf{d}\left(u\left(\omega+k \omega_{p}\right)\right)\right)\right\}}{\partial u(w)} . \\
& \frac{\partial u(\omega)}{\partial V_{\mathbf{x}}^{\perp}} \cdot \exp \left(-j\left(\omega+k \omega_{p}\right)\left(\delta_{M}+t_{0}\right)\right) \\
& =\left\{\mathbf{w}_{\mathbf{t}, \mathbf{M}}^{\dagger} \frac{\partial \mathbf{d}\left(u\left(\omega+k \omega_{p}\right)\right)}{\partial u(\omega)} \mathbf{w}_{\mathbf{r}, \mathbf{M}}^{\dagger} \mathbf{d}\left(u\left(\omega+k \omega_{p}\right)\right)+\right. \\
& \left.\mathbf{w}_{\mathbf{t}, \mathbf{M}}^{\dagger} \mathbf{d}\left(u\left(\omega+k \omega_{p}\right)\right) \mathbf{w}_{\mathbf{r}, \mathbf{M}}^{\dagger} \frac{\partial \mathbf{d}\left(u\left(\omega+k \omega_{p}\right)\right)}{\partial u(\omega)}\right\} \\
& \exp \left(-j\left(\omega+k \omega_{p}\right)\left(\delta_{M}+t_{0}\right)\right) \frac{\partial u(\omega)}{\partial V_{\mathbf{x}}^{\perp}}
\end{aligned}
$$

where $\frac{\partial \vec{d}\left(u\left(\omega+k \omega_{p}\right)\right)}{\partial u(\omega)}$ and $\frac{\partial u(\omega)}{\partial V_{\mathbf{x}}^{\perp}}$ are derived as

$$
\begin{gathered}
\frac{\partial \mathbf{d}\left(u\left(\omega+k \omega_{p}\right)\right)}{\partial u(\omega)}=\frac{\partial E_{e}(u)}{\partial u}\left[\begin{array}{c}
\exp \left(-j \beta x_{1} u\right) \\
\exp \left(-j \beta x_{2} u\right) \\
\vdots \\
\exp \left(-j \beta x_{N} u\right)
\end{array}\right]+E_{e}(u)\left[\begin{array}{c}
-j \beta x_{1} \exp \left(-j \beta x_{1} u\right) \\
-j \beta x_{2} \exp \left(-j \beta x_{2} u\right) \\
\vdots \\
-j \beta x_{N} \exp \left(-j \beta x_{N} u\right)
\end{array}\right] \\
\frac{\partial u(w)}{\partial V_{\mathrm{x}}^{\perp}}=\frac{-4 \beta\left(\omega+k \omega_{p}\right) V_{\mathrm{x}}^{\perp}\left(V_{\mathrm{g}}-V_{\mathrm{x}}\right)}{\left(2 \beta V_{\mathrm{rel}}^{2}\right)^{2}} \sqrt{\frac{1-\left(\frac{V_{\mathrm{rt}}^{\perp}}{V_{\mathrm{rel}}}\right)^{2}}{1-\left(\frac{\left(\omega+k \omega_{p}\right)}{2 \beta V_{\mathrm{rel}}}\right)^{2}}+\frac{\left(V_{\mathrm{g}}-V_{\mathrm{x}}\right)\left(V_{\mathrm{rel}}^{2}-V_{\mathrm{x}}^{\perp}\right) \sin (\theta)}{V_{\mathrm{rel}}^{4}}} \\
{\left[\frac{2 \beta\left(\omega+k \omega_{p}\right)\left(V_{\mathrm{g}}-V_{\mathrm{x}}\right) V_{\mathrm{x}}^{\perp}}{2 \beta V_{\mathrm{rel}}^{2}} \sqrt{\frac{\left(2 \beta V_{\mathrm{rel}}\right)^{2}-\left(\omega+k \omega_{p}\right)^{2}}{V_{\mathrm{rel}}^{2}-V_{\mathrm{rt}}^{\perp}}}\right.} \\
{\left[\frac{\cos ^{2}(\theta)\left(\left(2 \beta V_{\mathrm{rel}}\right)^{2}-\left(\omega+k \omega_{p}\right)^{2}\right)-2 \beta V_{\mathrm{rel}}\left(V_{\mathrm{rel}}^{2}-V_{\mathrm{rt}}^{\perp}\right)}{\left.\left(2 \beta V_{\mathrm{rel}}\right)^{2}-\left(\omega+k \omega_{p}\right)^{2}\right)^{2}}\right.}
\end{gathered}
$$


Thesis

where $\frac{\partial V_{\mathrm{r}}^{\perp}}{\partial V_{\mathrm{x}}^{\perp}}=\sin (\theta)$. The partial derivative $\frac{\partial \mathrm{M}_{\mathrm{k}}\left(V_{\mathrm{x}}^{\perp} ; \boldsymbol{\Theta}^{c}\right)}{\partial V_{\mathrm{x}}^{\perp}}$ is derived as

$$
\frac{\partial \mathrm{M}_{\mathrm{k}}\left(V_{\mathrm{x}}^{\perp} ; \boldsymbol{\Theta}^{c}\right)}{\partial V_{\mathrm{x}}^{\perp}}=j 2\left(\omega+k \omega_{p}\right) R_{0}\left(\frac{\sin (\theta) V_{\mathrm{rel}}^{2}-2 V_{\mathrm{rt}}^{\perp} V_{\mathrm{x}}}{\left(2 V_{\mathrm{rel}}^{2}\right)^{2}}\right) \exp \left(j\left(\omega+k \omega_{p}\right) \frac{R_{0} V_{\mathrm{rt}}^{\perp}}{2 V_{\mathrm{rel}}^{2}}\right) .
$$

The partial derivative $\frac{\partial \mathrm{N}_{\mathrm{k}}\left(V_{\mathrm{x}}^{\perp} ; \boldsymbol{\Theta}^{c}\right)}{\partial V_{\mathrm{x}}^{\perp}}$ is given as

$$
\begin{aligned}
\frac{\partial \mathrm{N}_{\mathrm{k}}\left(V_{\mathrm{x}}^{\perp} ; \boldsymbol{\Theta}^{c}\right)}{\partial V_{\mathrm{x}}^{\perp}}= & \frac{-2 j \beta R_{0}}{\sqrt{1-\left(\frac{V_{\mathrm{rt}}^{\perp}}{V_{\mathrm{rel}}}\right)^{2}} \sqrt{1-\frac{\left(\omega+k \omega_{p}\right)^{2}}{\left(2 \beta V_{\mathrm{rel}}\right)^{2}}}}\left[\left(1-\left(\frac{V_{\mathrm{rt}}^{\perp}}{V_{\mathrm{rel}}}\right)^{2}\right)\left(\frac{2 \beta V_{\mathrm{x}}^{\perp}\left(\omega+k \omega_{p}\right)^{2}}{\left(2 \beta V_{\mathrm{rel}}\right)^{2}}\right)\right. \\
& \left.+V_{\mathrm{x}}^{\perp}\left(1-\frac{\left(\omega+k \omega_{p}\right)^{2}}{\left(2 \beta V_{\mathrm{rel}}\right)^{2}}\right)\left(\frac{\left(1-\sin ^{2}(\theta)\right) V_{\mathrm{rel}}^{2}-\left(V_{\mathrm{rel}}^{2}-\left(V_{\mathrm{rt}}^{\perp}\right)^{2}\right)}{V_{\mathrm{rel}}^{4}}\right)\right] \\
& \exp \left(-2 j \beta R_{0} \sqrt{1-\left(\frac{V_{r t}^{\perp}}{V_{\text {rel }}}\right)^{2}} \sqrt{1-\frac{\left(\omega+k \omega_{p}\right)^{2}}{4 \beta^{2} V_{\text {rel }}^{2}}}\right) .
\end{aligned}
$$

\section{Validation of the Derivative}

The validation of the derivative was performed the same way as it was for the parameter $V_{\mathrm{x}}$ in Section 3.2.4. The numerical derivative was computed using the same method discussed previously. The result was then compared to the expression obtained analytically.

Figures 3.53 .7 show the derivative with respect to the parameter $V_{\mathrm{x}}^{\perp}$ at three different frequencies. The red curves show how derivative that was obtained analytically, and the green curve represents the derivative computed using the numerical method. The green curve is not apparent in these figures because the red curve lies almost exactly on top of them, which suggests a good agreement between the two derivatives. Figure 3.8 shows the error term, which was again computed by taking the difference between the two derivatives. The error term is quite low (on the order of $10^{-6}$ ), and similar to the error term in Figure 3.4, it oscillates due to the error in the computation of the numerical derivative. Similar to Equation 3.25, the error term is defined as the absolute value of the difference between the two derivatives and averaged over all the frequencies.

$$
\operatorname{Error} V_{\mathrm{x}}^{\perp}=\left\langle\left|\frac{\partial \mathbf{s}(\boldsymbol{\Theta})}{\partial V_{\mathrm{x}}^{\perp}}-\frac{\partial \mathbf{s}_{n}(\boldsymbol{\Theta})}{\partial V_{\mathrm{x}}^{\perp}}\right|\right\rangle
$$



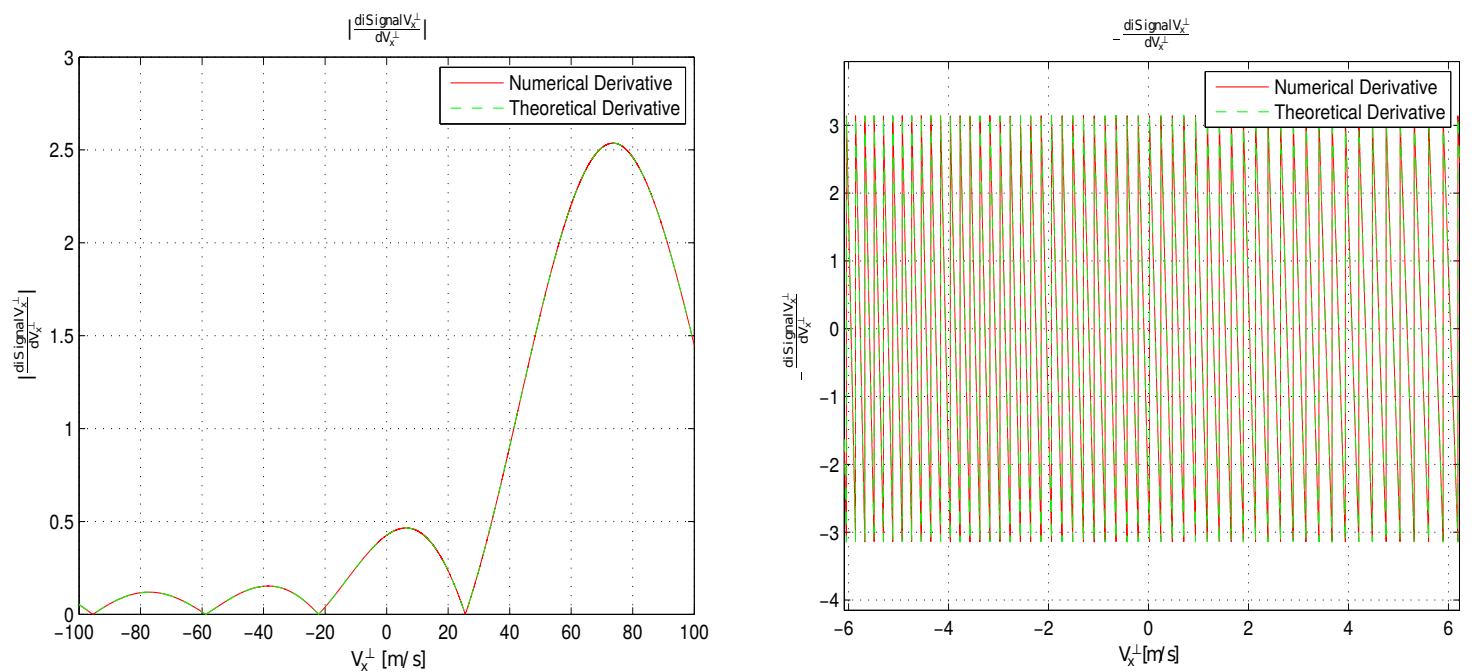

Figure 3.5: Signal derivative with respect to parameter $V_{\mathrm{x}}^{\perp}$ at $1 \mathrm{rad} / \mathrm{s}$.
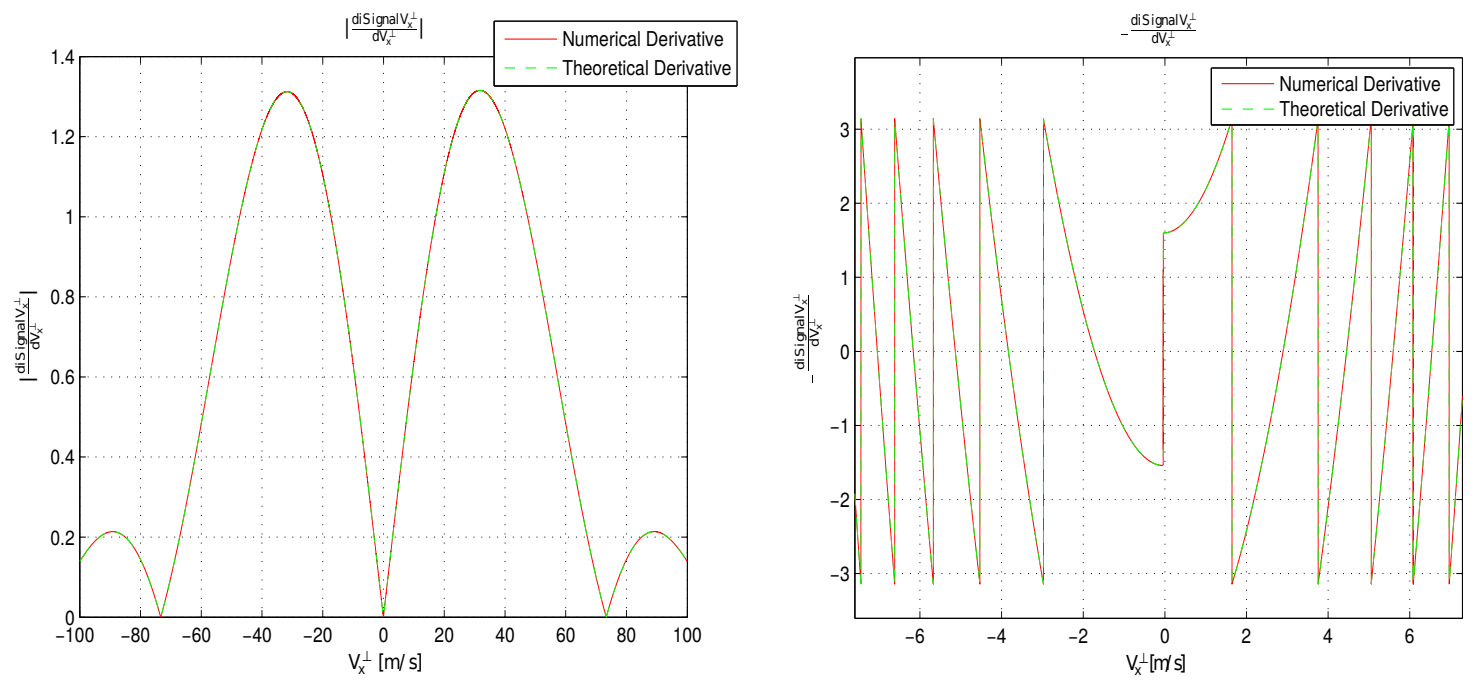

Figure 3.6: Signal derivative with respect to parameter $V_{\mathrm{x}}^{\perp}$ at $2 \mathrm{rad} / \mathrm{s}$. 

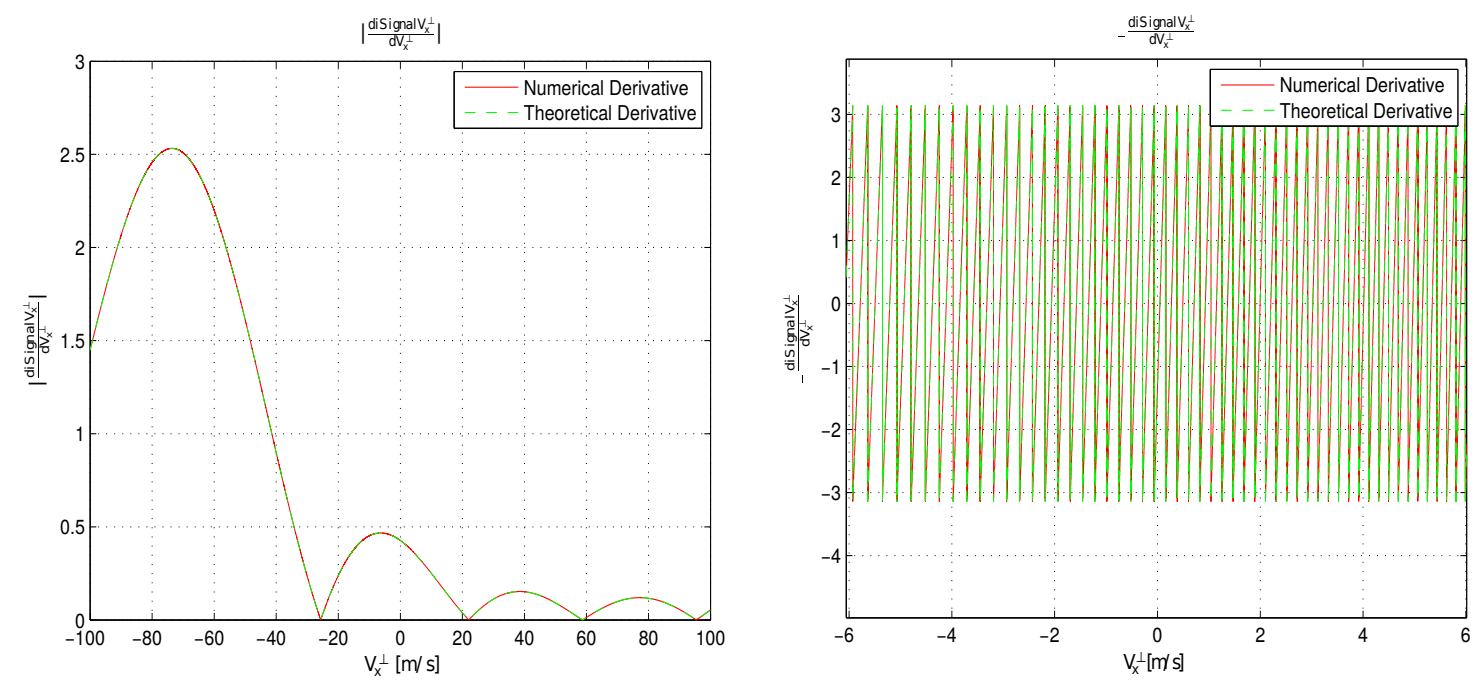

Figure 3.7: Signal derivative with respect to parameter $V_{\mathrm{x}}^{\perp}$ at $3 \mathrm{rad} / \mathrm{s}$.

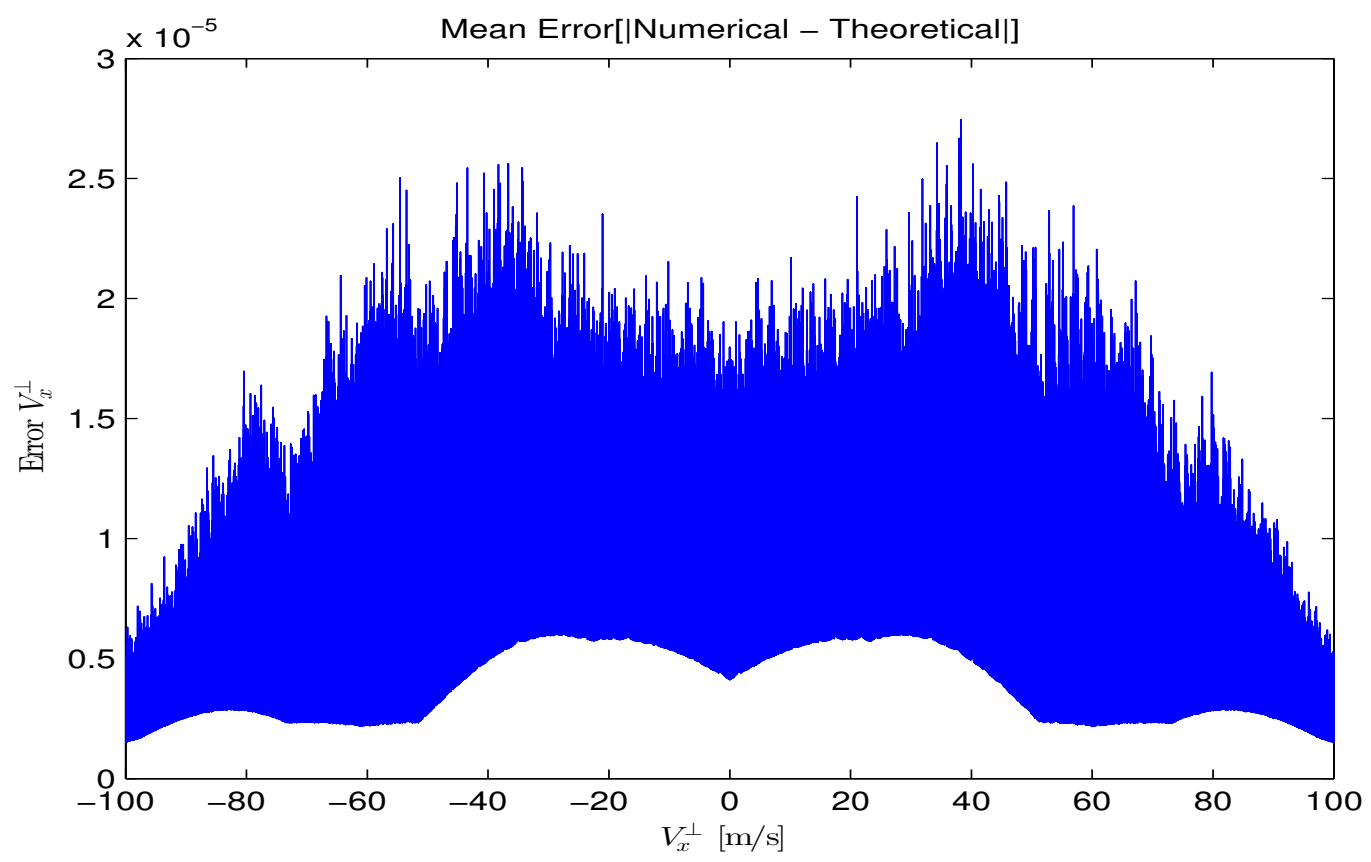

Figure 3.8: Error term for the numerical and theoretical derivative of $V_{\mathrm{x}}^{\perp}$. 


\section{Chapter 4}

\section{SMTI Performance Analysis}

A theoretical analysis of three SBR]SAR systems with respect to SMTI performance is provided and discussed in this chapter. As discussed in Chapter 1, CRLB can be used to test the performance and the feasibility of a system. In this thesis, the feasibility of a system is defined as the ability of the system to perform SMTI. The CRLB can provide the feasibility of the system by providing the variance estimates of different target parameters. If the variance estimates are within some given criterion then the system is accepted as feasible. In this chapter, the SMTI performance of three systems is analysed and compared using CRLB. These systems are RADARSAT-2, RCM, and a hypothetical system that is called "TestSat". RADARSAT-2 is an opertional system that is capable of SMTI. RCM is still in the development phase, and at the time of this writing (November 2015), its feasibility for SMTI is unknown. However, its aperture size and orbit parameters are similar to those of TerraSAR-X, which suggests that its SMTI performance will be much worse than RADARSAT-2 [13]. The system parameters for TestSat were chosen heuristically with the goal of reducing the transmitted power and aperture size while still obtaining SMTI performance, as provided by CRLB, that is comparable to RADARSAT-2. The orbit parameters for TestSat are similar to those of RCM, but its aperture size, transmit power, and gains were chosen to be theoretically better for SMTI, although not as optimal as RADARSAT-2. Reducing the transmitted power and aperture size should theoretically reduce the operational cost of the system since less transmitted power and a smaller aperture means lower power consumption.

The switching/toggling schemes that are considered in this thesis are based on the MODEX modes that are derived in [3], and presented in Appendix D. Note that only a subset of MODEX modes are considered in this thesis, however the analysis presented in 
this section would apply to the investigation of any other switching/toggling schemes.

\subsection{System Parameters}

RADARSAT-2 parameters are provided in Table 4.1, and the switching/toggling configurations are provided in Table 4.2. These parameters are identical to the parameters of RADARSAT-2 and can be found in $13,27,41$.

Table 4.1: RADARSAT-2 system parameters.

\begin{tabular}{|l|l|}
\hline Satellite Velocity & $7545 \mathrm{~m} / \mathrm{s}$ \\
\hline Wavelength & $5.5466 \mathrm{~cm}$ \\
\hline Maximum PRF & $3800 \mathrm{~Hz}$ \\
\hline Antenna Length & $15 \mathrm{~m}$ \\
\hline Orbit Altitude & $800 \mathrm{~km}$ \\
\hline Target Range & $950 \mathrm{~km}$ \\
\hline TX/RX Columns & 16 \\
\hline
\end{tabular}

Table 4.2: RADARSAT-2 switching configurations used for the simulations.

\begin{tabular}{|l|l|l|l|l|}
\hline & Modex-1 & $\begin{array}{l}\text { Modex-1 } \\
\text { Full PRF }\end{array}$ & Modex-2 3/4 & Modex-2 1/2 \\
\hline Number of TX Columns & 16 & 16 & 12 & 8 \\
\hline Number of RX Columns & 8 & 8 & 8 & 8 \\
\hline Number of Receive Channels & 2 & 2 & 4 & 4 \\
\hline PRF & $1800 \mathrm{~Hz}$ & $3750 \mathrm{~Hz}$ & $1875 \mathrm{~Hz}$ & $1875 \mathrm{~Hz}$ \\
\hline Transmit Power $\left(P_{t x}\right)$ & $36.6 \mathrm{~dB}$ & $36.6 \mathrm{~dB}$ & $35.4 \mathrm{~dB}$ & $33.6 \mathrm{~dB}$ \\
\hline Transmit Gain $\left(G_{t x}\right)$ & $49.6 \mathrm{~dB}$ & $49.6 \mathrm{~dB}$ & $48.4 \mathrm{~dB}$ & $46.6 \mathrm{~dB}$ \\
\hline Receive Gain $\left(G_{r x}\right)$ & $46.6 \mathrm{~dB}$ & $46.6 \mathrm{~dB}$ & $46.6 \mathrm{~dB}$ & $46.6 \mathrm{~dB}$ \\
\hline Noise Figure $\left(N_{f}\right)$ & $2.7 \mathrm{~dB}$ & $2.7 \mathrm{~dB}$ & $2.7 \mathrm{~dB}$ & $2.7 \mathrm{~dB}$ \\
\hline Losses $\left(L_{s}\right)$ & $5 \mathrm{~dB}$ & $5 \mathrm{~dB}$ & $5 \mathrm{~dB}$ & $5 \mathrm{~dB}$ \\
\hline Target Amplitude $\left(\sigma_{s}\right)$ & 0.0790 & 0.0790 & 0.0790 & 0.0790 \\
\hline Clutter Power $\left(\sigma_{c}\right)$ & 0.0933 & 0.0933 & 0.0933 & 0.0933 \\
\hline
\end{tabular}


The second system chosen has parameters similar to an individual satellite of RADARSAT Constellation Mission (RCM). As the name suggests, $\mathrm{RCM}$ is actually a constellation of three identical satellites, which will succeed RADARSAT-2 after their launch in 2018. The parameters listed in Tables 4.34 .4 were obtained from 42, 43], however, since this system is still under development, it is difficult to obtain all the parameters necessary for the simulations and analysis. The parameters that have been assumed have been indicated in their respective tables.

Table 4.3: RCM parameters.

\begin{tabular}{|l|l|}
\hline Satellite Velocity & $7588 \mathrm{~m} / \mathrm{s}$ \\
\hline Wavelength & $5.47 \mathrm{~cm}$ \\
\hline Maximum PRF & $6000 \mathrm{~Hz}$ \\
\hline Antenna Length & $8 \mathrm{~m}$ \\
\hline Orbit Altitude & $514 \mathrm{~km}$ \\
\hline Target Range & $600 \mathrm{~km}$ \\
\hline TX/RX Columns & 8 \\
\hline
\end{tabular}

Table 4.4: RCM Simulation Switching Parameters.

\begin{tabular}{|l|l|l|l|l|}
\hline & Modex-1 & $\begin{array}{l}\text { Modex-1 } \\
\text { Full PRF }\end{array}$ & Modex-2 3/4 & Modex-2 1/2 \\
\hline Number of TX Columns & 8 & 8 & 6 & 4 \\
\hline Number of RX Columns & 4 & 4 & 4 & 4 \\
\hline Number of Receive Channels & 2 & 2 & 4 & 4 \\
\hline PRF & $3000 \mathrm{~Hz}$ & $5950 \mathrm{~Hz}$ & $3000 \mathrm{~Hz}$ & $3000 \mathrm{~Hz}$ \\
\hline Transmit Power $\left(P_{t x}\right)$ & $33.6 \mathrm{~dB}$ & $33.6 \mathrm{~dB}$ & $32.4 \mathrm{~dB}$ & $31.7 \mathrm{~dB}$ \\
\hline Transmit Gain $\left(G_{t x}\right)$ & $46.6 \mathrm{~dB}$ & $46.6 \mathrm{~dB}$ & $45.4 \mathrm{~dB}$ & $43.6 \mathrm{~dB}$ \\
\hline Receive Gain $\left(G_{r x}\right)$ & $43.6 \mathrm{~dB}$ & $43.6 \mathrm{~dB}$ & $43.6 \mathrm{~dB}$ & $43.6 \mathrm{~dB}$ \\
\hline Noise Figure $\left(N_{f}\right)$ & $2.7 \mathrm{~dB}$ & $2.7 \mathrm{~dB}$ & $2.7 \mathrm{~dB}$ & $2.7 \mathrm{~dB}$ \\
\hline Losses $\left(L_{s}\right)$ & $5 \mathrm{~dB}$ & $5 \mathrm{~dB}$ & $5 \mathrm{~dB}$ & $5 \mathrm{~dB}$ \\
\hline Target Amplitude $\left(\sigma_{s}\right)$ & 0.0625 & 0.0625 & 0.0625 & 0.0625 \\
\hline Clutter Power $\left(\sigma_{c}\right)$ & 0.0117 & 0.0117 & 0.0117 & 0.0117 \\
\hline
\end{tabular}


The parameters for the TestSat system are listed in Table 4.5 and the switching configurations are provided in Table 4.6. Note that with the exception of the antenna length, this system is almost identical to $\mathrm{RCM}$.

Table 4.5: TestSAT parameters.

\begin{tabular}{|l|l|}
\hline Satellite Velocity & $7564 \mathrm{~m} / \mathrm{s}$ \\
\hline Wavelength & $5.47 \mathrm{~cm}$ \\
\hline Maximum PRF & $6000 \mathrm{~Hz}$ \\
\hline Antenna Length & $10 \mathrm{~m}$ \\
\hline Orbit Altitude & $600 \mathrm{~km}$ \\
\hline Target Range & $712 \mathrm{~km}$ \\
\hline TX/RX Columns & 12 \\
\hline
\end{tabular}

Table 4.6: TestSAT simulation switching parameters.

\begin{tabular}{|l|l|l|l|l|}
\hline & Modex-1 & $\begin{array}{l}\text { Modex-1 } \\
\text { Full PRF }\end{array}$ & Modex-2 3/4 & Modex-2 1/2 \\
\hline Number of TX Columns & 12 & 12 & 8 & 6 \\
\hline Number of RX Columns & 6 & 6 & 6 & 6 \\
\hline Number of Receive Channels & 2 & 2 & 4 & 4 \\
\hline PRF & $3000 \mathrm{~Hz}$ & $5950 \mathrm{~Hz}$ & $3000 \mathrm{~Hz}$ & $3000 \mathrm{~Hz}$ \\
\hline Transmit Power $\left(P_{t x}\right)$ & $35.0 \mathrm{~dB}$ & $35.0 \mathrm{~dB}$ & $34.0 \mathrm{~dB}$ & $32.4 \mathrm{~dB}$ \\
\hline Transmit Gain $\left(G_{t x}\right)$ & $48.4 \mathrm{~dB}$ & $48.4 \mathrm{~dB}$ & $46.2 \mathrm{~dB}$ & $45.4 \mathrm{~dB}$ \\
\hline Receive Gain $\left(G_{r x}\right)$ & $45 \mathrm{~dB}$ & $45 \mathrm{~dB}$ & $45 \mathrm{~dB}$ & $45 \mathrm{~dB}$ \\
\hline Noise Figure $\left(N_{f}\right)$ & $2.7 \mathrm{~dB}$ & $2.7 \mathrm{~dB}$ & $2.7 \mathrm{~dB}$ & $2.7 \mathrm{~dB}$ \\
\hline Losses $\left(L_{s}\right)$ & $5 \mathrm{~dB}$ & $5 \mathrm{~dB}$ & $5 \mathrm{~dB}$ & $5 \mathrm{~dB}$ \\
\hline Target Amplitude $\left(\sigma_{s}\right)$ & 0.0868 & 0.0868 & 0.0868 & 0.0868 \\
\hline Clutter Power $\left(\sigma_{c}\right)$ & 0.0174 & 0.0174 & 0.0174 & 0.0174 \\
\hline
\end{tabular}

The parameters listed in the preceding tables were used to compute the CRLBs derived in Chapter 3. Note that CRLB depends on the target RCS and clutter reflectivity, which affect the signal-to-noise and clutter-to-noise ratios (SNR/CNR), respectively. Expressions for the integrated SNR and CNR are given in Equations E.14b and E.18, in 
Appendix E. To compute the CRLBs, the target RCS and clutter reflectivity were chosen such that the integrated SNR and CNR for the MODEX-2 3/4 configuration provided a reasonable value, which depending on the system was approximated between $15 \mathrm{~dB}$ and $22 \mathrm{~dB}$ (see SCNR plots below). The target $\left(\sigma_{s}\right)$ and clutter $\left(\sigma_{c}\right)$ amplitudes were then chosen to be the nominal values of the single pulse SNR and CNR, respectively. To make the simulations manageable, the same amplitude values were used for all the switchiSng/toggling configurations as the one derived for MODEX-2 3/4 1 .

\subsection{SMTI Performance Analysis}

This section presents the SMTI performance of the three systems discussed previously with ISTAP. For SMTI, the target parameter of interest is the across-track velocity $\left(V_{\mathrm{x}}^{\perp}\right)$, since it affects the estimation of the target radial velocity (see Equation 2.28), and the target azimuth position error [4]. Therefore, SMTI performance is investigated by computing the CRLB for the across-track velocity component of the target, and the ISTAP clutter filter that was discussed in Section 2.3.4.

The RADARSAT-2 ISTAP clutter filters for various MODEX modes are provided in Figure 4.1. The results are similar to those presented in [1,13. The figure shows that the best clutter suppression performance is achieved by the MODEX-1 FULL PRF clutter filter. This can be seen from the highest gain and the narrowest notch of the magenta curve that corresponds to this mode.

\footnotetext{
${ }^{1}$ In realistic scenarios, the signal and clutter amplitudes would be different for each configuration.
} 


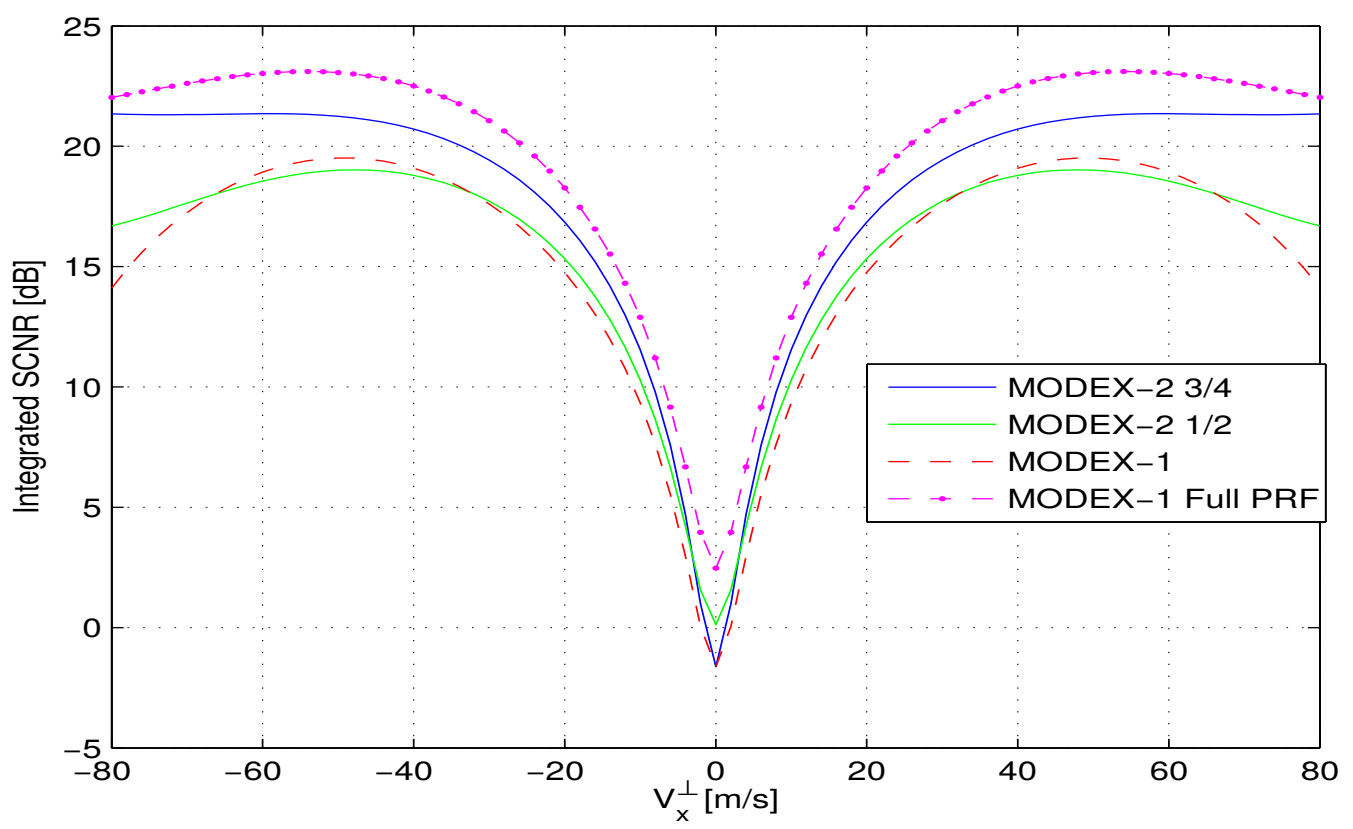

Figure 4.1: RADARSAT-2 ISTAP clutter filters for various MODEX modes.

The CRLB for various MODEX modes are presented in Figure 4.2. Note that for MODEX-1 FULL PRF mode, the estimated standard deviation of the across-track velocity in the CRLB plot is almost twice compared to the four channel (MODEX-2) modes. This shows that even though the MODEX-1 FULL PRF mode provides the best clutter suppression, it may not be ideal for SMTI as it provides a high standard deviation in the across-track speed estimate, relative to other modes. For a system designer, the goal for optimal SMTI is to minimize the standard deviation seen in the CRLB plot of Figure 4.2, and to maximize the clutter suppression performance in the filters in Figure 4.1. Comparing the two figures, it can be seen that the MODEX-2 3/4 provides the best clutter suppression and moving target gain from the four channel modes. MODEX-2 1/2 has a slightly lower estimated standard deviation, but the high SNCR loss in its clutter filter means that it doesn't represent the most optimal trade-off. The best trade-off between the SNCR and the estimated standard deviation, according to the two figures, is achieved by the MODEX-2 3/4 mode. The superior performance of MODEX-2 3/4 for RADARSAT-2 in terms low estimation variance was also concluded in [14. 


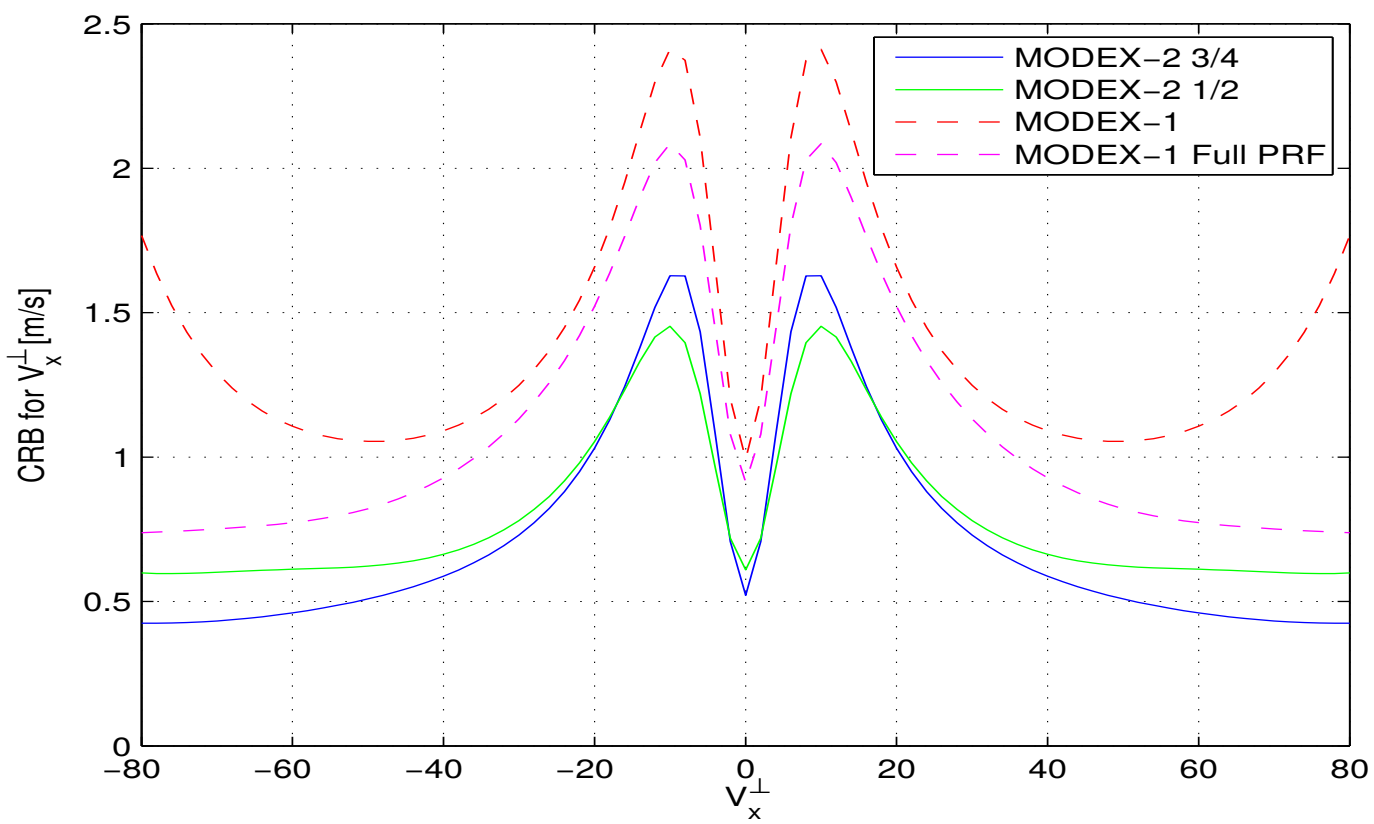

Figure 4.2: RADARSAT-2 CRLB for across-track velocity estimation, plotted as the standard-deviation in $\mathrm{m} / \mathrm{s}$ versus $V_{\mathrm{x}}^{\perp}$.

The RCM clutter filters for various MODEX modes are provided in Figure 4.3. The figure shows that RCM provides a much poorer SMTI performance than RADARSAT-2. This can be seen through the wide notches and substantially lower gains of the high-pass filters for all the modes. 


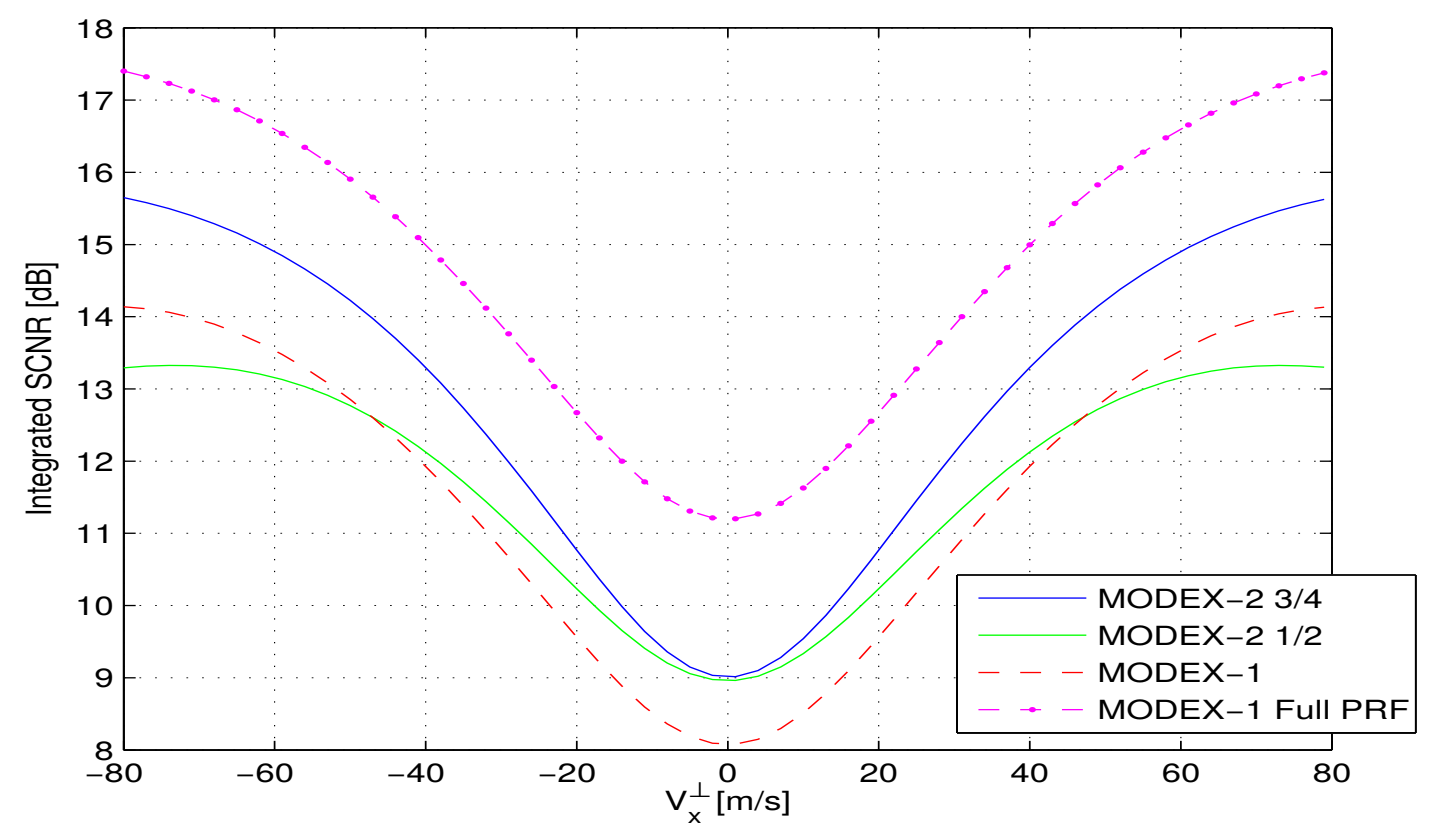

Figure 4.3: RCM ISTAP clutter filters for various MODEX modes.

RCM CRLB plots for the various MODEX modes are provided in Figure 4.4. The CRLB provides a substantially higher standard deviation than RADARSAT-2. Similar to TerraSAR-X, this substantial loss in performance is mainly attributed to a much smaller aperture size [13]. Comparing Figure 4.3 and Figure 4.4, it can be seen that MODEX-2 $3 / 4$ mode provides the best trade-off between the SNCR loss and the estimation standard deviation for RCM. However, even if the CRLB is reached, the SMTI would be quite poor compared to RADARSAT-2. 


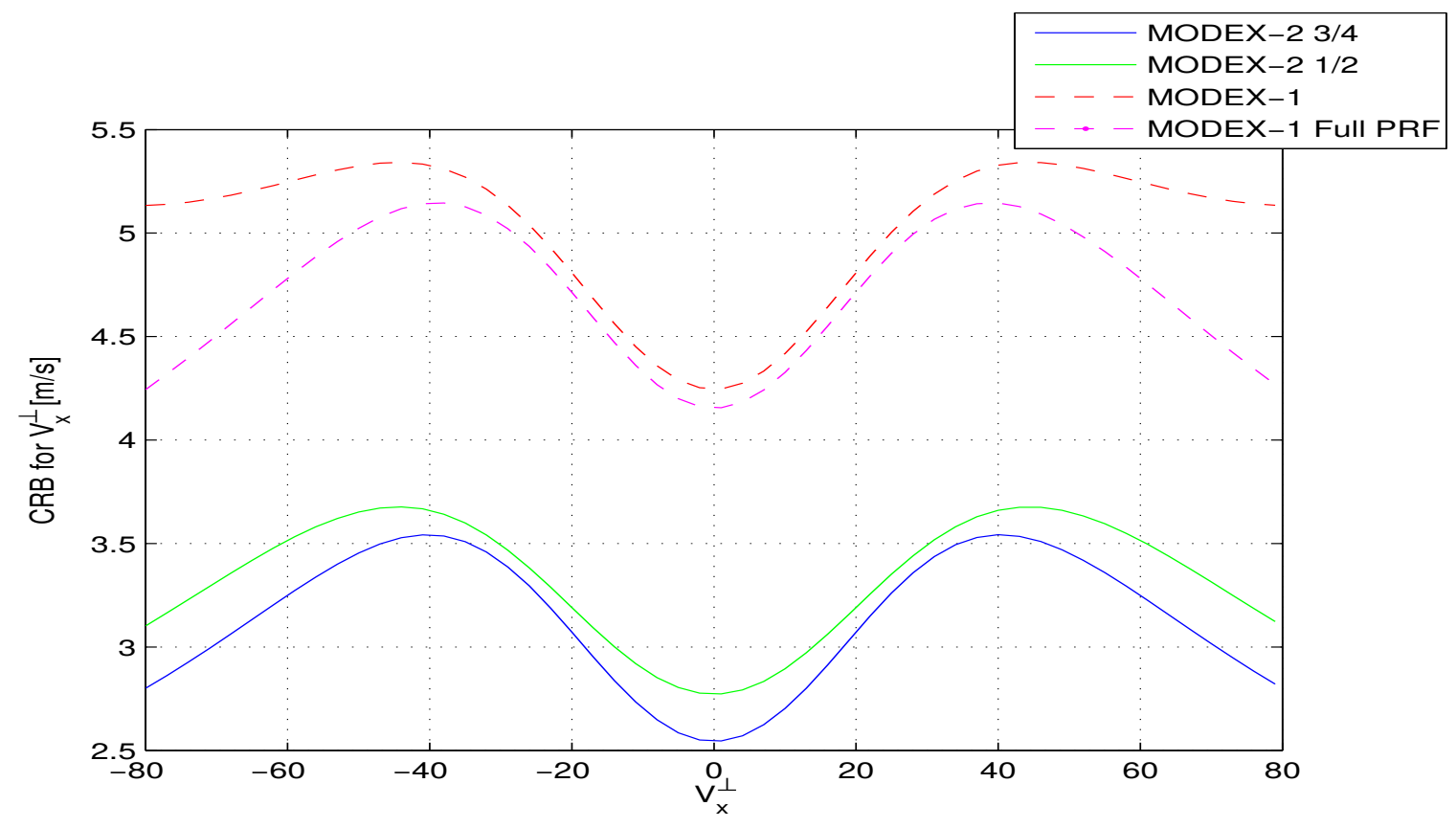

Figure 4.4: RCM CRLB for across-track velocity estimation, plotted as the standarddeviation in $m / s$ versus $V_{\mathrm{x}}^{\perp}$.

TestSAT clutter filters for the different MODEX modes are provided in Figure 4.5. Due to a larger aperture size, the SNCR loss is not as profound as that of RCM and the gains for the different modes are similar to RADARSAT-2. The clutter suppression is comparable to RADARSAT-2, although not as good due to the wider notches for all the modes. 


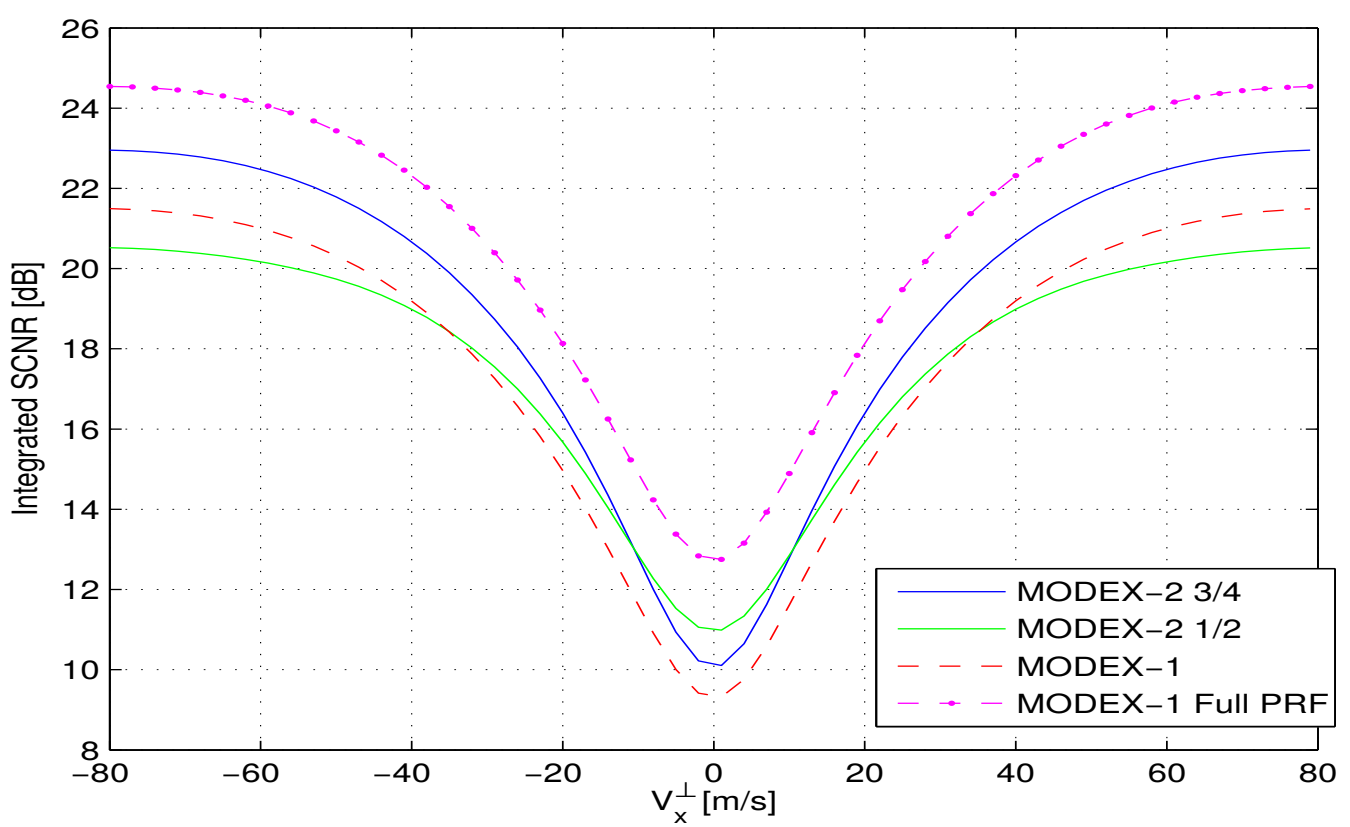

Figure 4.5: TestSAT ISTAP clutter filters for various MODEX modes.

Lastly, TestSAT CRLB plots for the different MODEX modes are provided in Figure 4.6 below. These plots show that the across-track velocity standard deviation is just slightly larger than RADARSAT-2 for all the modes, and the SMTI performance is quite comparable. Comparing the two figures for TestSAT, it can be seen that MODEX-2 3/4 provides the most optimal trade-off between the SNCR loss and the estimated standard deviation. 


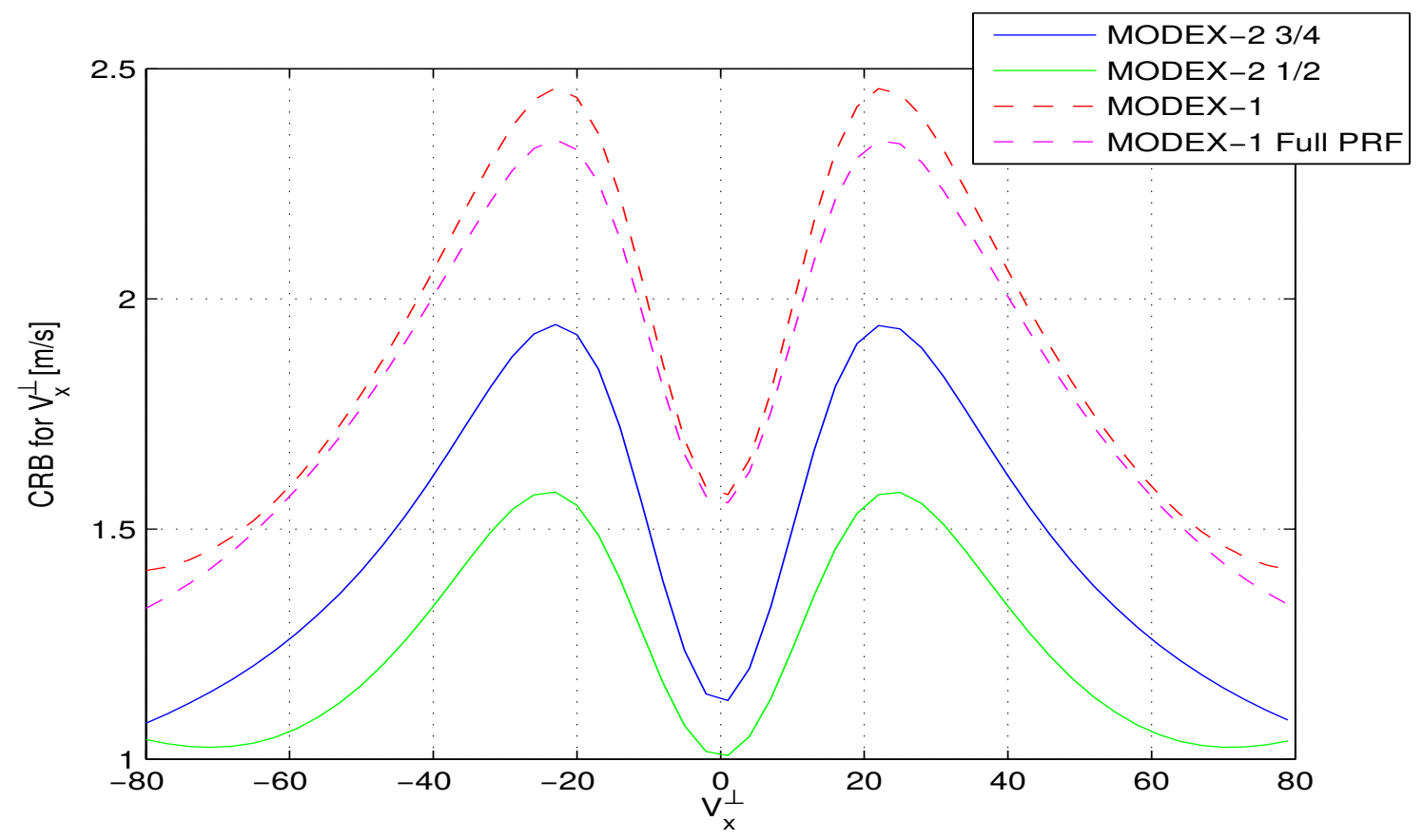

Figure 4.6: TestSAT CRLB for across-track velocity estimation, plotted as the standarddeviation in $m / s$ versus $V_{\mathrm{x}}^{\perp}$.

Some key observations can be noted from the CRLB and clutter filter plots of the three systems. Firstly, the across-track velocity estimation standard deviation decreases for MODEX-1 when the PRF is increased. In the simulations, the PRF was almost doubled for the three systems, which corresponds to about a $3 \mathrm{~dB}$ gain of the clutter suppression filter. This gain is theoretically justified because increasing the PRF increases the number of samples that can be obtained from the same point target, which increases the integration gain $N_{i n t}$ in Equation E.13. Another key observation is that increasing the number of channels also decreases the across-track velocity estimation standard deviation. This can be seen from the lower CRLB curves for MODEX-2 in the plots. This observation can also be theoretically justified because increasing the number of channels provides a higher spatial diversity (i.e., higher degrees of freedom) [2 4]. Higher spatial diversity means more measurements available for parameter estimation. 


\section{Chapter 5}

\section{Conclusions}

In this thesis, a background of space-based SAR and SMTI is presented. The multichannel signal model for ISTAP is derived for space-borne systems in satellite ECEF coordinate system. Concepts of moving target detection methods related to ATI, DPCA, and STAP are discussed. The CRLB for ISTAP are derived by finding the derivatives with respect to moving target parameters, and the results are confirmed using numerical analysis. A theoretical performance analysis using CRLB for three different systems over a variety of switching/toggling schemes is presented.

\subsection{Thesis Contributions}

The main contributions of this thesis are presented in Chapters 3 and 4 . In Chapter 3, CRLB of a statistical model of the signal over a parameter set is derived. The signal model utilizes Gaussian distributions for clutter and noise models. The CRLBs are derived by finding the derivatives of the model over the target parameter set. The derivatives over some of the parameters are non-trivial and were therefore confirmed using numerical analysis. The results of the numerical analysis have also been provided. The CRLB in itself is a valuable tool that provides a necessary but not sufficient design baseline for system parameters (transmitted power, number of transmit/receive columns, etc).

In Chapter 4, an analysis of three SBR-SAR systems is provided. The goal of the analysis was two-fold: i) to find the SMTI performance limits of realistic systems over different switching/toggling configurations, and ii) to use CRLB as a benchmark tool to determine if it is possible to have a system that consumes less power than an existing 
system and provides a comparable or better SMTI performance. The conclusions of this analysis are as follows

- Increasing the number of channels, i.e., higher signal diversity, reduces the acrosstrack velocity estimation variance. However, it also increases the SCNR loss of the signal. This trade-off is due to the fact that a lower estimation variance is achieved by increasing the signal diversity, which typically uses a smaller antenna aperture. However, an increase in diversity comes at a cost of a reducation in signal-to-noise ratio since the signal is transmitted with less power. For SMTI, the most desirable antenna configuration provides the most optimal trade-off between the SCNR loss and estimation variance. CRLB can used to find the most optimal trade-off.

- Increasing the system pulse repetition frequency (PRF) reduces the estimation variance and increases the gain of the high-pass clutter filter.

- From the MODEX modes that were investigated, the four channel 3/4 mode (MODEX-2 3/4) provided the best trade-off between the SNCR loss and the estimation standard deviation for all three systems.

- A system with larger aperture generally provides better performance due to high transmit power and gains. However, these systems are costly and are therefore rarely developed. In non-classified space, only RADARSAT-2 is capable of SMTI due to its large aperture $(15 \mathrm{~m})$. The analysis in Chapter 4 shows that a system deployed in an orbit $600 \mathrm{~km}$, and with an aperture length of $10 \mathrm{~m}$, which is about two-thirds of RADARSAT-2 and only $2 \mathrm{~m}$ larger than RCM, can provide a performance that is comparable to RADARSAT-2.

\subsection{Suggestions for Future Work}

Research in space-based SAR SMTI is still incomplete and ongoing. Suggestions for future work that would benefit the development of SBR SMTI are as follows

- CRLB derived in this report used a signal model for the "standard" SAR strip-map mode. The model can be extended to include Scan-SAR, Spotlight-SAR, and other SAR modes to find the performance under other configurations.

- The theoretical results presented in this thesis should be compared to results of detectors from real datasets to see if real detectors reach the limits provided by 
CRLB. This would provide a further confirmation of the results provided in this thesis.

- The CRLB analysis can be extended to include the bias sensitivity in the presence of the biased estimators 44].

- New switching/toggling configurations can be designed by optimizing CRLB expressions presented in this thesis [20]. 


\section{References}

[1] D. Cerutti-Maori and I. Sikaneta, "Optimum GMTI Processing for Space-based SAR/GMTI Systems - Simulation Results," in 8th European Conference on Synthetic Aperture Radar (EUSAR 2010), June 2010, pp. 1-4.

[2] M. Dragosevic, W. Burwash, and S. Chiu, "Detection and Estimation With RADARSAT-2 Moving-Object Detection Experiment Modes," IEEE Transactions on Geoscience and Remote Sensing, vol. 50, no. 9, pp. 3527-3543, Sept 2012.

[3] S. Chiu, C. Livingstone, I. C. Sikaneta, C. H. Gierull, and P. Beaulne, "RADARSAT2 Moving Object Detection Experiment (MODEX)." in 2008 IEEE International Geoscience and Remote Sensing Symposium (IGARSS'08), 2008, pp. 13-16.

[4] D. Cerutti-Maori and I. Sikaneta, "Optimum GMTI Processing for Space-based SAR/GMTI Systems - Theoretical Derivation," in 8th European Conference on Synthetic Aperture Radar (EUSAR 2010), June 2010, pp. 1-4.

[5] J. H. Ender, D. Cerutti-Maori, and W. Burger, "Radar antenna architectures and sampling strategies for space based moving target recognition," in 2005 IEEE International Geoscience and Remote Sensing Symposium (IGARSS'05), vol. 4. IEEE, 2005, pp. 2921-2924.

[6] F. M. Henderson and A. J. Lewis, Principles and applications of imaging radar. Manual of remote sensing, volume 2. John Wiley and sons, 1998.

[7] I. G. Cumming and F. H.-C. Wong, Digital processing of synthetic aperture radar data: algorithms and implementation. Artech House, 2005.

[8] C. H. Gierull, "Moving Target Detection with Along-Track SAR Interferometry," Aerospace Radar and Navigation section, Defence R\&D Canada - Ottawa, Tech. Rep. TR 2002-084, August 2002. 
[9] S. Chiu and C. Livingstone, "A comparison of displaced phase centre antenna and along-track interferometry techniques for RADARSAT-2 ground moving target indication," Canadian Journal of Remote Sensing, vol. 31, no. 1, pp. 37-51, 2005.

[10] R. Klemm, Principles of space-time adaptive processing. IET, 2002, no. 159.

[11] — _ Applications of space-time adaptive processing. IET, 2004, vol. 14.

[12] M. C. Wicks, M. Rangaswamy, R. Adve, and T. B. Hale, "Space-time adaptive processing: a knowledge-based perspective for airborne radar," Signal Processing Magazine, IEEE, vol. 23, no. 1, pp. 51-65, 2006.

[13] J. Ender, C. Gierull, and D. Cerutti-Maori, "Improved space-based moving target indication via alternate transmission and receiver switching," IEEE Transactions on Geoscience and Remote Sensing, vol. 46, no. 12, pp. 3960-3974, Dec 2008.

[14] D. Cerutti-Maori, I. Sikaneta, and C. H. Gierull, "Optimum SAR/GMTI processing and its application to the radar satellite RADARSAT-2 for traffic monitoring," IEEE Transactions on Geoscience and Remote Sensing, vol. 50, no. 10, pp. 3868-3881, 2012 .

[15] Y. C. Eldar and G. Kutyniok, Compressed sensing: theory and applications. Cambridge University Press, 2012.

[16] M. A. Richards, J. Scheer, and W. A. Holm, Principles of modern radar: basic principles. SciTech Pub., 2010.

[17] I. Cumming and J. R. Bennett, "Digital processing of SEASAT SAR data," in 1979 IEEE International Conference on Acoustics, Speech, and Signal Processing, IEEE International Conference on (ICASSP 1979)., vol. 4. IEEE, 1979, pp. 710-718.

[18] A. Moreira, P. Prats-Iraola, M. Younis, G. Krieger, I. Hajnsek, and K. Papathanassiou, "A tutorial on synthetic aperture radar," Geoscience and Remote Sensing Magazine, IEEE, vol. 1, no. 1, pp. 6-43, March 2013.

[19] S. Chiu and M. Dragošević, "Moving target indication via RADARSAT-2 multichannel synthetic aperture radar processing," EURASIP Journal on Advances in Signal Processing, vol. 2010, p. 7, 2010. 
Conclusions

[20] C. H. Gierull, I. Sikaneta, and D. Cerutti-Maori, "Improved SAR-GMTI via Optimized Crame-Rao Bound," in 10th European Conference on Synthetic Aperture Radar (EUSAR 2014), June 2014, pp. 1-4.

[21] J. C. Curlander and R. N. McDonough, Synthetic Aperture Radar. John Wiley \& Sons, 1991.

[22] R. Raney, "A comment on Doppler FM rate," International Journal of Remote Sensing, vol. 8, no. 7, pp. 1091-1092, 1987.

[23] — - "Synthetic Aperture Imaging Radar and Moving Targets," IEEE Transactions on Aerospace and Electronic Systems, vol. AES-7, no. 3, pp. 499-505, May 1971.

[24] M. Pettersson, "Extraction of moving ground targets by a bistatic ultra-wideband SAR," IEE Proceedings - Radar, Sonar and Navigation, vol. 148, no. 1, pp. 35-40, Feb 2001.

[25] S. Chiu and C. Gierull, "Multi-channel receiver concepts for RADARSAT-2 ground moving target indication," 6th European Conference on Synthetic Aperture Radar (EUSAR 2006), 2006.

[26] J. Uher, J. Mennitto, and D. McLaren, "Design concepts for the Radarsat-2 SAR antenna," in Antennas and Propagation Society International Symposium, 1999. IEEE, vol. 3. IEEE, 1999, pp. 1532-1535.

[27] P. A. Fox, A. P. Luscombe, and A. A. Thompson, "RADARSAT-2 SAR modes development and utilization," Canadian Journal of Remote Sensing, vol. 30, no. 3, pp. 258-264, 2004.

[28] C. E. Livingstone and A. A. Thompson, "The moving object detection experiment on RADARSAT-2," Canadian journal of remote sensing, vol. 30, no. 3, pp. 355-368, 2004 .

[29] C. W. Chen, "Performance assessment of along-track interferometry for detecting ground moving targets."

[30] A. Moccia and G. Rufino, "Spaceborne along-track SAR interferometry: Performance analysis and mission scenarios," IEEE Transactions on Aerospace and Electronic Systems, vol. 37, no. 1, pp. 199-213, 2001. 
[31] C. H. Gierull, "Statistical analysis of multilook SAR interferograms for CFAR detection of ground moving targets," IEEE Transactions on Geoscience and Remote Sensing, vol. 42, no. 4, pp. 691-701, 2004.

[32] S. Stergiopoulos, Advanced Signal Processing: Theory and Implementation for Sonar, Radar, and Non-Invasive Medical Diagnostic Systems. CRC Press, 2009.

[33] I. C. Sikaneta, "Detection of ground moving objects with synthetic aperture radar," Ph.D. dissertation, University of Ottawa (Canada)., 2004.

[34] E. Stockburger and D. Held, "Interferometric moving ground target imaging," in 1995 IEEE International Radar Conference. IEEE, 1995, pp. 438-443.

[35] D. Cerutti-Maori and I. Sikaneta, "A Generalization of DPCA Processing for Multichannel SAR/GMTI Radars," IEEE Transactions on Geoscience and Remote Sensing, vol. 51, no. 1, pp. 560-572, Jan 2013.

[36] H. L. V. Trees, Detection, Estimation, and Modulation Theory: Radar-Sonar Signal Processing and Gaussian Signals in Noise. Krieger Publishing Co., Inc., 1992.

[37] R. A. Monzingo and T. W. Miller, Introduction to adaptive arrays. SciTech Publishing, 1980.

[38] B. Bradie, A friendly introduction to numerical analysis. Pearson Prentice Hall Upper Saddle River, NJ, 2006.

[39] B. P. Flannery, S. Teukolsky, W. H. Press, and W. T. Vetterling, "Numerical Recipes in $\mathrm{C}++, " 2002$.

[40] C. Ridders, "Accurate computation of $\mathrm{f}(\mathrm{x})$ and $\mathrm{f}(\mathrm{x}) \mathrm{f}(\mathrm{x})$," Advances in Engineering Software (1978), vol. 4, no. 2, pp. 75-76, 1982.

[41] Z. Ali, G. Kroupnik, G. Matharu, J. Graham, I. Barnard, P. Fox, and G. Raimondo, "Radarsat-2 space segment design and its enhanced capabilities with respect to radarsat-1," Canadian Journal of Remote Sensing, vol. 30, no. 3, pp. 235-245, 2004. [Online]. Available: http://dx.doi.org/10.5589/m03-077

[42] J. Colinas, G. Séguin, and P. Plourde, "Radarsat constellation, moving toward implementation," in 2010 IEEE International Geoscience and Remote Sensing Symposium (IGARSS'2010). IEEE, 2010, pp. 3232-3235. 
[43] A. A. Thompson, "Innovative capabilities of the radarsat constellation mission," in 8th European Conference on Synthetic Aperture Radar (EUSAR 2010). VDE, 2010, pp. 1-3.

[44] G. E. Newstadt and A. O. Hero, "Cramér Rao Lower Bound analysis of multichannel SAR with spatially varying, correlated noise," in SPIE Defense+ Security. International Society for Optics and Photonics, 2014, pp. 90 930L-90 930L.

[45] W. Torge, Geodesy. Walter de Gruyter, 2001.

[46] O. Montenbruck and E. Gill, Satellite orbits: models, methods and applications. Springer Science \& Business Media, 2000.

[47] V. Trees and L. Harry, Detection, Estimation, and Modulation Theory-Part lDetection, Estimation, and Linear Modulation Theory. John Wiley \& Sons, 2001. 


\section{Appendix A}

\section{Space-based Geometry}

The SAR signal model derived in section 2.2 is based on an imaging geometry model of the satellite and target in Earth-Centerd, Earth-Fixed (ECEF) coordinate system. The model of the imaging geometry, which forms the basis for the analysis in this report, is derived in this section.

\section{A.1 Coordinate Systems}

The coordinate systems that are relevant to satellite orbit analysis are listed in this section. The coordinate systems discussed below are either inertial or fixed relative to the earth or satellite. These coordinate systems are commonly used space-based applications, and more details can be found in [45,46]. The systems are also illustrated in Figure A.1.

\begin{tabular}{|l|l|l|}
\hline System & Symbol & Description \\
\hline Antenna Coordinate System & A & $\begin{array}{l}\text { This system has its origin at the cen- } \\
\text { tre of the radar antenna, an x-axis that } \\
\text { aligns with the vector from the aft of } \\
\text { the antenna to the fore of the antenna, } \\
\text { a z-axis that points in the direction } \\
\text { from the centre of the earth to the satel- } \\
\text { lite, and a y-axis that completes the } \\
\text { right-handed coordinate system. }\end{array}$ \\
\hline
\end{tabular}




\begin{tabular}{|c|c|c|}
\hline $\begin{array}{l}\text { Earth-Centered, Earth-Fixed Coordi- } \\
\text { nate System (ECEF) }\end{array}$ & $\mathrm{E}$ & $\begin{array}{l}\text { The system has its origin at the cen- } \\
\text { tre of mass of the Earth. The x- } \\
\text { axis passes through } 0^{\circ} \text { latitude (Equa- } \\
\text { tor) and } 0^{\circ} \text { longitude (Greenwich), the } \\
\text { z-axis points through the North pole } \\
\text { and the y-axis completes the right- } \\
\text { handed coordinate system. This coor- } \\
\text { dinate system rotates with the earth } \\
\text { and therefore, coordinates of a point } \\
\text { fixed on the surface of the earth do not } \\
\text { change. }\end{array}$ \\
\hline Earth-Centered Inertial System & $\mathbf{I}$ & $\begin{array}{l}\text { The system has its origin at the cen- } \\
\text { tre of mass of Earth. The x-axis in- } \\
\text { tersects the equator in the direction of } \\
\text { the sun, the z-axis points through the } \\
\text { North pole, and the y-axis completes } \\
\text { the right-handed coordinate system. In } \\
\text { this coordinate system, the earth ro- } \\
\text { tates so that the x-axis intersects the } \\
\text { equator in the direction of the sun. }\end{array}$ \\
\hline Satellite Coordinate System & $\mathrm{S}$ & $\begin{array}{l}\text { The system has its origin at the cen- } \\
\text { tre of mass of the satellite. The x- } \\
\text { axis points in the direction of the satel- } \\
\text { lite motion in the inertial coordinate } \\
\text { system, a z-axis that points in the di- } \\
\text { rection from the centre of mass of the } \\
\text { earth to the satellite, and a y-axis that } \\
\text { completes the right-handed coordinate } \\
\text { system. }\end{array}$ \\
\hline
\end{tabular}




\begin{tabular}{|l|l|l|}
\hline Satellite ECEF Coordinate System & D & $\begin{array}{l}\text { This coordinate system has its origin } \\
\text { at the centre of mass of Earth. The } \mathrm{x}^{-} \\
\text {axis is aligned in the satellite velocity } \\
\text { direction in ECEF coordinate system. } \\
\text { The z-axis is aligned along the vector } \\
\text { from the centre of mass the earth to } \\
\text { the centre of mass of the satellite, and } \\
\text { the y-axis completes the right-handed } \\
\text { coordinate system. Note that this coor- } \\
\text { dinate system has the same orientation } \\
\text { as Satellite Coordinate System (S), but } \\
\text { its origin is at the centre of the earth. }\end{array}$ \\
\hline
\end{tabular}

\section{A.2 Coordinate Transformations}

In this section, the transformation between the coordinate systems listed in previous is discussed.

\section{A.2.1 Transformation from I to $\mathrm{E}$}

In system $\mathbf{I}$, the $\mathrm{x}$-axis points in the direction toward the sun. In system $\mathbf{E}$, the $\mathbf{x}$-axis points towards $0^{\circ}$ latitude and $0^{\circ}$ longitude. In both systems, the z-axis points towards the north pole. Therefore, a transformation from system $\mathbf{I}$ to system $\mathbf{E}$ can be seen as a rotation about the z-axis by an angle $\omega_{e} t$, where $\omega_{e}$ is angular rotation rate of the earth as shown in Figure A.1. A position vector $\mathbf{X}_{\mathbf{I}}(t)$ in system $\mathbf{I}$ is transformed into a position vector $\mathbf{X}_{\mathbf{E}}(t)$ in system $\mathbf{E}$ using a rotation matrix $\mathbf{R}_{\mathbf{E I}}(t)$.

$$
\mathbf{X}_{\mathbf{E}}(t)=\underbrace{\left[\begin{array}{ccc}
\cos \omega_{e} t & \sin \omega_{e} t & 0 \\
-\sin \omega_{e} t & \cos \omega_{e} t & 0 \\
0 & 0 & 1
\end{array}\right]}_{\mathbf{R}_{\mathbf{E I}}(t)} \mathbf{X}_{\mathbf{I}}(t)
$$




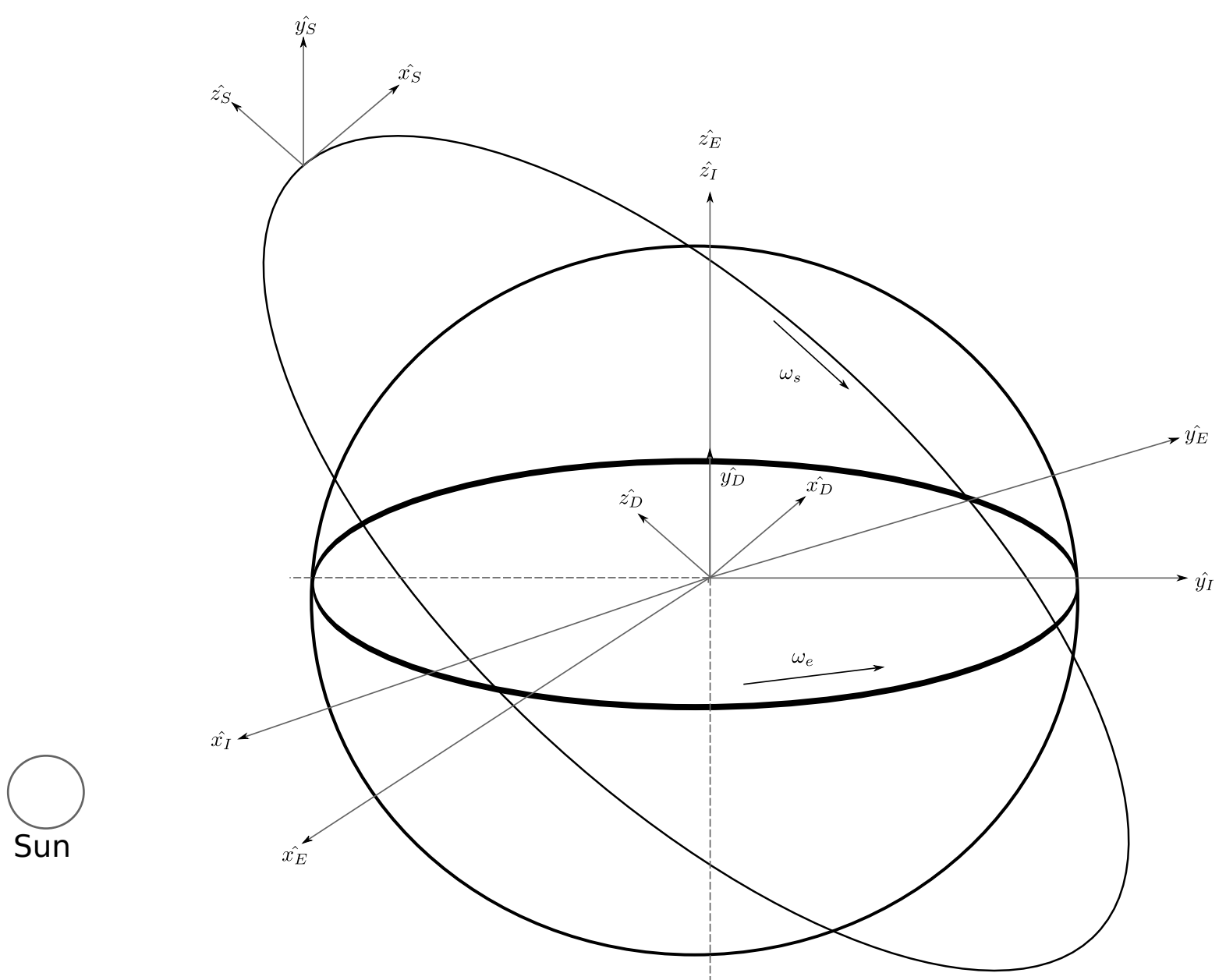

Figure A.1: Common coordinate systems used in remote sensing. 


\section{A.2.2 Transformation from I to $\mathrm{S}$}

In system $\mathbf{S}$, the z-axis points in the direction from the centre of the Earth to the satellite. Due to the satellite orbit, this coordinate system rotates relative to system $\mathbf{I}$ according to the angular speed of the satellite $\left(\omega_{s}\right)$ about the $\mathrm{x}$-axis. There is also a rotation about the y-axis due to the inclination $\left(\theta_{i}\right)$ of the orbit, as shown in Figure A.1. Furthermore, there is a rotation of $\frac{-\pi}{2}$ about the $\mathrm{z}$-axis, in order to complete the right-handed system. Finally, the origin of the system is shifted by $\Delta_{S}$. A position vector $\mathbf{X}_{I}(t)$ in system $\mathbf{I}$ is transformed to a position vector $\mathbf{X}_{S}(t)$ in system $\mathbf{S}$ as follows:

$$
\mathbf{X}_{\mathbf{S}}(t)=\underbrace{\left[\begin{array}{ccc}
\cos \left(\frac{-\pi}{2}\right) & \sin \left(\frac{-\pi}{2}\right) & 0 \\
-\sin \left(\frac{-\pi}{2}\right) & \cos \left(\frac{-\pi}{2}\right) & 0 \\
0 & 0 & 1
\end{array}\right]\left[\begin{array}{ccc}
1 & 0 & 0 \\
0 & \cos \left(\omega_{s} t\right) & -\sin \left(\omega_{s} t\right) \\
0 & \sin \left(\omega_{s} t\right) & \cos \left(\omega_{s} t\right)
\end{array}\right]\left[\begin{array}{ccc}
\cos \left(\pi-\theta_{i}\right) & 0 & \sin \left(\pi-\theta_{i}\right) \\
0 & 1 & 0 \\
-\sin \left(\pi-\theta_{i}\right) & 0 & \cos \left(\pi-\theta_{i}\right)
\end{array}\right]}_{\mathbf{R}_{\mathbf{S I}}(t)} \mathbf{X}_{\mathbf{I}}(t)-\underbrace{\left[\begin{array}{c}
\Delta x_{s} \\
\Delta y_{s} \\
\Delta z_{s}
\end{array}\right]}_{\Delta s}
$$

\section{A.2.3 Transformation from $\mathrm{S}$ to $\mathrm{A}$}

In system $\mathbf{A}$, the x-axis is shifted so that it is aligned from the aft of the antenna to the fore of the antenna. Therefore, there is a shift in the $\mathrm{x}-\mathrm{y}$ plane relative to system $\mathbf{S}$. This shift is determined by the yaw angle about the z-axis. A position vector $\mathbf{X}_{\mathbf{S}}(t)$ in system $\mathbf{S}$ is transformed into a position vector $\mathbf{X}_{\mathbf{A}}(t)$ in system $\mathbf{A}$ as follows:

$$
\mathbf{X}_{\mathbf{E}}(t)=\underbrace{\left[\begin{array}{ccc}
\cos \Theta_{y}(t) & \sin \Theta_{y}(t) & 0 \\
-\sin \Theta_{y}(t) & \cos \Theta_{y}(t) & 0 \\
0 & 0 & 1
\end{array}\right]}_{\mathbf{R}_{\mathbf{A} \mathbf{S}}(t)} \mathbf{X}_{\mathbf{I}}(t)
$$

In Equation A.3. $\Theta_{y}(t)$ is the yaw steering function. This function is designed so that the antenna is always aligned in along-track direction in the coordinate system $\mathbf{E}$ [41].

\section{A.2.4 Transformation from A to $\mathrm{E}$}

Using relations in sections A.2.1 to A.2.3, the transformation from system $\mathbf{A}$ to system E can be determined as follows:

$$
\mathbf{X}_{\mathbf{E}}(t)=\mathbf{R}_{\mathbf{E I}}(t) \mathbf{R}_{\mathbf{I S}}(t)\left[\mathbf{R}_{\mathbf{S A}}(t) \mathbf{X}_{\mathbf{A}}+\Delta_{S}\right]
$$


Note that Equation A.4 implies that the origin of the satellite in system $\mathbf{E}$ is

$$
\Delta_{E}(t)=\mathbf{R}_{\mathbf{E I}}(t) \mathbf{R}_{\mathbf{I S}}(t) \Delta_{S},
$$

and the velocity at this point in system $\mathbf{E}$ is given by

$$
\dot{\Delta}_{E}(t)=\left[\frac{\mathrm{d} \mathbf{R}_{\mathbf{E I}}(t)}{\mathrm{d} t} \mathbf{R}_{\mathbf{I S}}(t)+\mathbf{R}_{\mathbf{E I}}(t) \frac{\mathrm{d} \mathbf{R}_{\mathbf{I S}}(t)}{\mathrm{d} t}\right] \Delta_{S},
$$

where the magnitude of this velocity vector is given by

$$
V_{a}(t)=\left|\dot{\Delta}_{E}(t)\right|=R_{s} \omega_{s} \sqrt{1+\frac{\omega_{e}^{2}}{\omega_{s}^{2}} \sin ^{2} \theta_{i} \sin ^{2} \omega_{s} t-2 \frac{\omega_{e} \omega_{s}}{\cos } \theta_{i}+\frac{\omega_{e}^{2}}{\omega_{s}^{2}} \cos ^{2} \theta_{i}}
$$

\section{A.3 Satellite Position and Velocity Vectors}

In this section, the expressions for position and velocity of the antenna phase-centre in system $\mathbf{D}$ are derived. According to the definition of system D listed in Table A.1, the $\mathrm{X}$-axis is aligned in the satellite velocity direction in the ECEF coordinate system, the z-axis is aligned along the vector from the centre of the earth to the centre of mass of the satellite, and the y-axis completes the right-handed coordinate system. Using this definition, the unit vectors at any fixed instant of time $\left(t=t_{0}\right)$, that transform any vector in system $\mathbf{E}$ to a vector in system $\mathbf{D}$ can be written as follows

$$
\begin{aligned}
\hat{\mathbf{x}}_{\mathbf{D}}\left(t_{0}\right) & =\frac{\dot{\Delta}_{E}\left(t_{0}\right)}{\left|\dot{\Delta}_{E}\left(t_{0}\right)\right|} \\
\hat{\mathbf{y}}_{\mathbf{D}}\left(t_{0}\right) & =\frac{\Delta_{E}\left(t_{0}\right) \times \dot{\Delta}_{E}\left(t_{0}\right)}{\left|\Delta_{E}\left(t_{0}\right)\right|\left|\dot{\Delta}_{E}\left(t_{0}\right)\right|} \\
\hat{\mathbf{z}}_{\mathbf{D}}\left(t_{0}\right) & =\frac{\Delta_{E}\left(t_{0}\right)}{\left|\Delta_{E}\left(t_{0}\right)\right|}
\end{aligned}
$$

The coordinate of the $n^{t h}$ antenna phase-centre in antenna coordinate system is given by

$$
\mathbf{x}_{\mathbf{A}}^{n}=\left[\begin{array}{c}
r_{n} \\
0 \\
0
\end{array}\right]
$$


and the $n^{\text {th }}$ phase-centre in ECEF coordinates is located at

$$
\mathbf{x}_{\mathbf{E}}^{n}(t)=\Delta_{E}(t)+r_{n} \frac{\dot{\Delta}_{E}(t)}{\left|\dot{\Delta}_{E}(t)\right|},
$$

where $\Delta_{E}(t)$ is the position of the satellite, $r_{n}$ the distance to the $n^{t h}$ phase-centre in system A, and $\frac{\dot{\Delta}_{E}(t)}{\left|\dot{\Delta}_{E}(t)\right|}$ is the unit-vector that transforms the distance to ECEF coordinates. The location of the $n^{\text {th }}$ phase-centre in system $\mathbf{D}$ is given by

$$
\mathbf{x}_{\mathbf{D}}^{n}(t)=\left[\begin{array}{l}
\hat{\mathbf{x}}_{\mathbf{D}}\left(t_{0}\right) \cdot \mathbf{x}_{\mathbf{E}}^{n}(t) \\
\hat{\mathbf{y}}_{\mathbf{D}}\left(t_{0}\right) \cdot \mathbf{x}_{\mathbf{E}}^{n}(t) \\
\hat{\mathbf{z}}_{\mathbf{D}}\left(t_{0}\right) \cdot \mathbf{x}_{\mathbf{E}}^{n}(t)
\end{array}\right] .
$$

A Taylor series expansion around $t=t_{0}$ is used to expand Equation A.11 in order to obtain an approximation that is more feasible for the analysis presented in this report. The Taylor expansion is as follows

$$
\mathbf{x}_{\mathbf{D}}^{n}(t) \approx \mathbf{x}_{\mathbf{D}}^{n}\left(t_{0}\right)+\frac{\mathrm{d} \mathbf{x}_{\mathbf{D}}^{n}\left(t_{0}\right)}{\mathrm{d} t}\left(t-t_{0}\right)+\frac{1}{2} \frac{\mathrm{d}^{2} \mathbf{x}_{\mathbf{D}}^{n}\left(t_{0}\right)}{\mathrm{d} t^{2}}\left(t-t_{0}\right)^{2},
$$

where the contribution from the higher-order derivatives is assumed to be insignificant. Before deriving the derivatives in Equation A.12, the following relations should be noted

$$
\begin{aligned}
\Delta_{E}(t) \cdot \dot{\Delta}_{E}(t) & =0, \\
\dot{\Delta}_{E}(t) \cdot \ddot{\Delta}_{E}(t) & =0, \\
\frac{\mathrm{d}\left|\Delta_{E}(t)\right|}{\mathrm{d} t} & \approx 0 .
\end{aligned}
$$

Furthermore, the following notation is adopted

$$
\begin{array}{r}
V_{\mathrm{a}}=\left|\Delta_{E}\left(t_{0}\right)\right|, \\
-a_{a} \hat{\mathbf{z}}_{\mathbf{D}}\left(t_{0}\right)=\ddot{\Delta}_{E}\left(t_{0}\right) .
\end{array}
$$

where $V_{\mathrm{a}}$ is the average magnitude of the velocity vector, and $a_{a}$ is the magnitude of the gravitational acceleration towards the earth. Note that in order for the satellite to remain in orbit, the centripetal acceleration should balance the gravitational acceleration 
towards the earth

$$
\begin{array}{r}
a_{a} \approx \frac{V_{\mathrm{a}}^{2}}{R_{s}}, \\
a_{a} \approx \frac{G M_{\text {earth }}}{R_{s}^{2}},
\end{array}
$$

where $M_{\text {earth }}$ is the mass of the earth, and $G$ denotes the universal gravitational constant.

The time derivatives in Equation A.12 can now be derived using Equation A.11 and the relations A.13a, A.14b

$$
\begin{gathered}
\mathbf{x}_{\mathbf{D}}^{n}\left(t_{0}\right)=\left[\begin{array}{c}
r_{n} \\
0 \\
R_{s}
\end{array}\right], \\
\frac{\mathrm{d} \mathbf{x}_{\mathbf{D}}^{n}\left(t_{0}\right)}{\mathrm{d} t}=\left[\begin{array}{c}
V_{\mathrm{a}} \\
0 \\
\frac{-r_{n} a_{a}}{V_{\mathrm{a}}}
\end{array}\right], \\
\frac{\mathrm{d}^{2} \mathbf{x}_{\mathbf{D}}^{n}\left(t_{0}\right)}{\mathrm{d} t^{2}}=\left[\begin{array}{c}
0 \\
0 \\
-a_{a}
\end{array}\right] .
\end{gathered}
$$

The satellite position vector for the $n^{\text {th }}$ phase-centre in system $\mathbf{D}$ is now derived by substituting the expressions in equations A.16a A.16c in the Taylor expansion of Equation A.12

$$
\mathbf{x}_{\mathbf{D}}^{n}(t) \approx\left[\begin{array}{c}
V_{\mathrm{a}} t+r_{n} \\
0 \\
r_{s}-r_{n} \frac{a_{a}}{V_{\mathrm{a}}} t-\frac{a_{a}}{2} t^{2}
\end{array}\right],
$$

where the time origin has been moved: $t \rightarrow t-t_{0}$. The velocity vector for the $n^{t h}$ phase-centre in system $\mathbf{D}$ is derived by taking the time derivative of the position vector

$$
\dot{\mathrm{x}}_{\mathbf{D}}^{n}(t) \approx\left[\begin{array}{c}
V_{\mathrm{a}} \\
0 \\
-r_{n} \frac{a_{a}}{V_{\mathrm{a}}}-\frac{a_{a}}{t}
\end{array}\right]
$$




\section{A.4 Moving Target}

The imaging geometry of Figure A.1 is used to derive the model for a moving target. The target is located at broadside and is shown in red on the surface of the earth.

Motion parameters of the target are given by the along-track velocity $\left(V_{x}\right)$, which is parallel to satellite velocity vector, and across-track velocity $\left(V_{x}^{\perp}\right)$, which is in the crossrange direction. The target acceleration is assumed to be zero during the imaging time, and is therefore ignored in the subsequent analysis. In order to derive an expression for the target position vector in ECEF satellite coordinate system (D), the component of the across-track velocity along the $\hat{y}_{D}$ and $\hat{z}_{D}$ axis must be computed. This is shown in equations A.19 and A.20 below.

$$
\begin{array}{r}
V_{y}=V_{x}^{\perp} \cos (\alpha(\theta)) \\
V_{y}=V_{x}^{\perp} \cos \left(-\left(\frac{\pi}{2}-\theta+\phi(\theta)\right)\right) \\
V_{y}=V_{x}^{\perp} \sin (\theta+\phi(\theta) \\
V_{z}=V_{x}^{\perp} \sin (\alpha(\theta)) \\
V_{z}=V_{x}^{\perp} \sin \left(-\left(\frac{\pi}{2}-\theta+\phi(\theta)\right)\right) \\
V_{z}=-V_{x}^{\perp} \cos (\theta+\phi(\theta))
\end{array}
$$

In SAR analysis, it is also beneficial to define radial velocity, $V_{r t}^{\perp}$, which is the component of target velocity projected along the line-of-sight (LOS) vector. From Figure A.2, the relationship between radial velocity and the across-track velocity is as follows:

$$
V_{r t}^{\perp}=V_{x}^{\perp} \sin (\theta)
$$

In terms of $V_{y}$ and $V_{z}$, the radial velocity is defined as:

$$
V_{r t}^{\perp}=V_{y} \cos (\phi(\theta))+V_{z} \sin (\phi(\theta))
$$




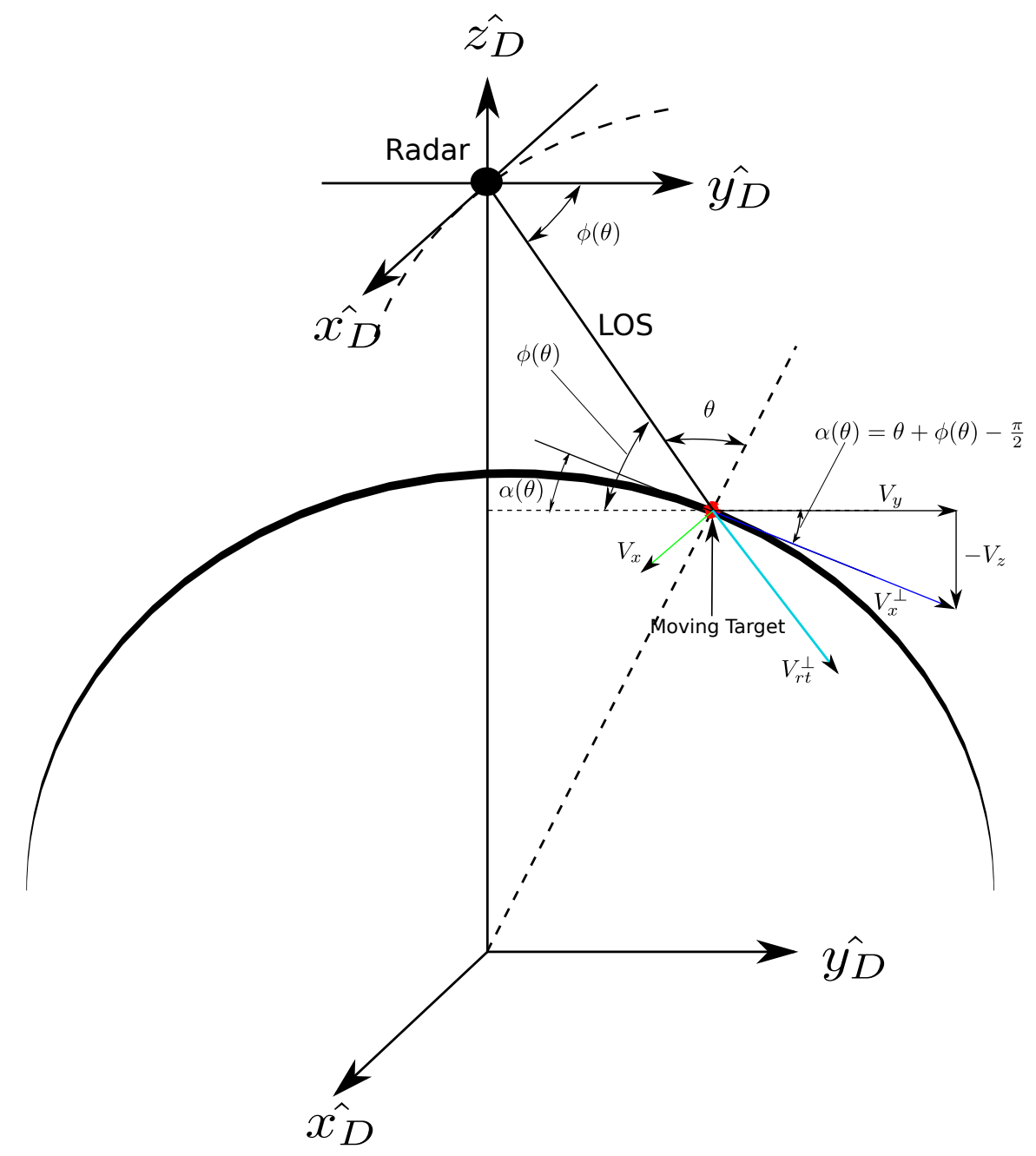

Figure A.2: Satellite imaging geometry. Moving target, shown in red, is located at broadside (zero squint). The target velocity is decomposed into an along-track component, $V_{x}$ (shown in green), which is parallel to satellite velocity vector, and the across-track component, $V_{x}^{\perp}$ (shown in blue). The across-track component can be further decomposed into $V_{y}$ and $V_{z}$. 
Using A.19 and A.20, the position vector of an arbitrary moving target located at broadside is follows:

$$
\mathbf{x}_{\mathbf{t}}(t)=\left[\begin{array}{c}
V_{\mathrm{x}} t \\
y_{0}(\theta)+V_{y} t \\
z_{0}(\theta)+V_{z} t
\end{array}\right]
$$

\section{A.4.1 Antenna Look Direction}

To derive an expression for the antenna look direction, a reference must be made to the Antenna Coordinate System (A). The antenna is steered in yaw so that it is aligned in the direction of the satellite velocity in ECEF Coordinate System (E). The velocity vector in System A is given by Equation A.24. The velocity vector in System D, at the centre of the antenna, can be determined by substituting $r_{n}=0$ in Equation A.18.

$$
\dot{\mathbf{x}}_{\mathbf{A}}(t)=\left[\begin{array}{c}
\sqrt{V_{a}^{2}+a_{a}^{2} t^{2}} \\
0 \\
0
\end{array}\right]
$$

Transformation from System D to System A involves a rotation around the y-axis in the $\mathrm{x}-\mathrm{z}$ plane. The transformation matrix for this rotation about the $\mathrm{y}$-axis can be written as follows

$$
\mathbf{R}_{\mathbf{A D}}(t)=\left[\begin{array}{ccc}
\cos \Theta(t) & 0 & \sin \Theta(t) \\
0 & 1 & 0 \\
-\sin \Theta(t) & 0 & \cos \Theta(t)
\end{array}\right]
$$

where the rotation angle, $\Theta(t)$, is the yaw-steering function. Expressions for $\cos \Theta(t)$ and $\sin \Theta(t)$ are found by noting the rotation angle between the two coordinate sysems as illustrated in Figure A.3.

$$
\begin{aligned}
& \cos \Theta(t)=\frac{V_{a}}{\sqrt{V_{a}^{2}+a_{a}^{2} t^{2}}} \\
& \sin \Theta(t)=\frac{-a_{a} t}{\sqrt{V_{a}^{2}+a_{a}^{2} t^{2}}}
\end{aligned}
$$

The antenna look-direction is derived as follows

$$
\mathbf{u}(t)=\mathbf{R}_{\mathbf{A D}} \frac{\left[\mathbf{x}_{\mathbf{D}}^{n}-\mathbf{x}_{\mathbf{t}}\right]}{\left|\mathbf{x}_{\mathbf{D}}^{n}-\mathbf{x}_{\mathbf{t}}\right|}
$$




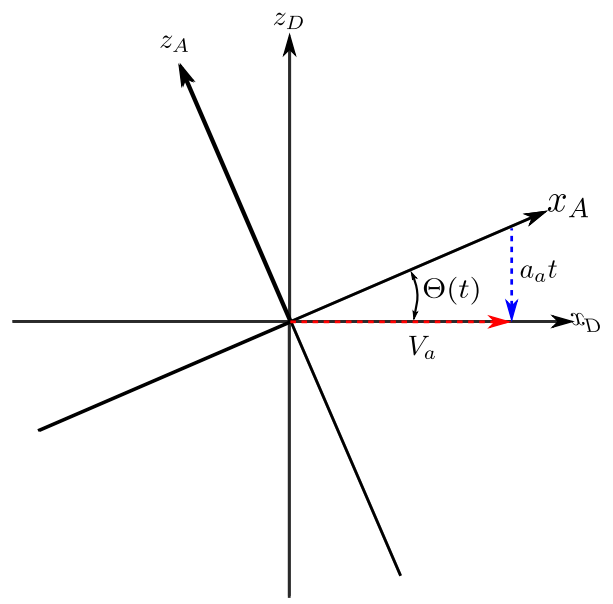

Figure A.3: The rotation angle $(\Theta(t))$ between sytems A and D.

The numerator in Equation A.28 is expanded as follows

$$
\left[\mathbf{x}_{\mathbf{D}}^{n}-\mathbf{x}_{\mathbf{t}}\right]=\left[\begin{array}{c}
\left(V_{\mathrm{x}}-V_{\mathrm{a}}\right) t \\
y_{0}+V_{y} t \\
z_{0}-R_{s}+V_{z} t+\frac{a_{a} t^{2}}{2}
\end{array}\right]
$$

The expression $\left|\mathbf{x}_{\mathbf{D}}^{n}-\mathbf{x}_{\mathbf{t}}\right|$ is expanded as follows

$$
R_{n}(t)=\left|\mathbf{x}_{\mathbf{D}}^{n}-\mathbf{x}_{\mathbf{t}}\right|=\sqrt{V_{\mathrm{rel}}^{2} t^{2}+2 V_{\mathrm{rt}}^{\perp} R_{0} t+R_{0}^{2}}
$$

The look direction vector can now be found by substituting equations A.25, A.29, and A.30 into Equation A.28 as follows

$$
\mathbf{u}(t)=\frac{1}{R_{n}(t)}\left[\begin{array}{c}
\left(V_{\mathrm{x}}-V_{\mathrm{g}}-\frac{a_{a} V_{z}}{V_{\mathrm{a}}}-\frac{a_{a}^{2} t}{2 V_{\mathrm{a}}}\right) \frac{V_{\mathrm{a}} t}{\sqrt{V_{\mathrm{a}}^{2}+a_{a}^{2} t^{2}}} \\
y_{0}+V_{y} t \\
\left(\left(V_{\mathrm{x}}-\frac{V_{\mathrm{a}}}{2}\right) a_{a} t^{2}+V_{\mathrm{a}} V_{z} t+V_{\mathrm{a}}\left(z_{0}-R_{s}\right)\right) \frac{1}{\sqrt{V_{\mathrm{a}}^{2}+a_{a}^{2} t^{2}}}
\end{array}\right]
$$

A useful approximation of Equation A.31 can be obtained by eliminating all the $a_{a}$ since the value of $a_{a}$ is about three orders of magnitude smaller than $V_{\mathrm{a}}$. This approximation is given in Equation A.32 below.

$$
\mathbf{u}(t) \approx \frac{1}{R_{n}(t)}\left[\begin{array}{c}
\left(V_{\mathrm{x}}-V_{\mathrm{g}}\right) t \\
y_{0}+V_{y} t \\
\left(V_{z} t+\left(z_{0}-R_{s}\right)\right)
\end{array}\right]
$$


In literature, for strip-map SAR, the first-order Taylor approximation around $t=0$ of the azimuth angle directional cosine or scalar "look direction" is most commonly used to $2,13,13$. This approximation is given in Equation A.33, and used throughout the thesis.

$$
u(t) \approx \frac{V_{\mathrm{x}}-V_{\mathrm{g}}}{R_{0}}
$$




\section{Appendix B}

\section{Azimuth Parameters from Antenna Theory}

Some of the important prameters of a SAR signal, such as azimuth bandwidth and azimuth resolution, are derived from antenna theory. In this chapter, the azimuth resolution of a real aperture is derived from fundamental antenna concepts. Then, using the same concepts, the resolution of the processed synthetic aperture is derived.

\section{B.1 Real Aperture}

Consider a linear array antenna consisting on $2 N+1$ isotropic and equally spaced radiating elements, as shown in Figure B.1. The elements are assumed to be aligned along the azimuth direction with the center element placed at the origin of the axis. Assume

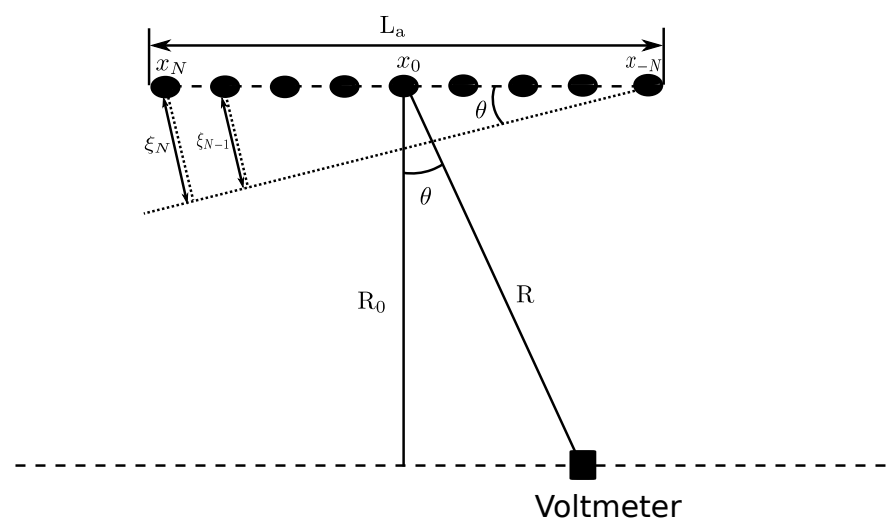

Figure B.1: Far-field radiation of a linear array antenna. 
far-field conditions so that the rays reaching the voltmeter from each element are parallel. The radiation pattern is found by summing the contribution from each element, which depends on the path length to each element 1 .

The array transmits a plane wave at an angle $\theta$ from the line of elements. A "wavefront" depicting a plane wave is shown by the dotted line in Figure B.1. Rays perpendicular to the wavefront show the direction of travel of the wave. The path length for the $N^{t h}$ element is given by distance $\xi_{N}=x_{N} \sin (\theta)$, where $x_{N}$ is the distance from the reference element, which is placed at origin, to the $N^{t h}$ element. Note that the path length to the reference element is 0 . The radiation pattern is then derived from the sum of each radiating element in the array

$$
\mathrm{A}(\theta)=\sum_{n=-N}^{N} \exp \left(-j \beta x_{n} \sin (\theta)\right)
$$

where $\beta$ is the angular wave number, which is equal to $\frac{2 \pi}{\lambda}$. The beam pattern for the antenna is derived by noting that the antenna is constructed using a very large number of such elements that are spaced very closely together. The sum in the above expression then converges to the Fourier integral that gives the one-way antenna beam pattern

$$
\mathrm{A}(\theta)=\int_{-L_{a} / 2}^{+L_{a} / 2} \exp \left(-j \beta x_{n} \sin (\theta)\right) d x_{n}=L_{a} \operatorname{sinc}\left(L_{a} \frac{\sin (\theta)}{\lambda}\right),
$$

The beam pattern of the antenna is therefore a sinc function with maximum at broadside 2) The 3-dB beamwidth associated with this function is

$$
\theta_{b w} \approx 0.866 \frac{\lambda}{L_{a}},
$$

where the approximation comes after using small-angle approximation of $\sin (\theta) \approx \theta$ in Equation B.2. The real aperture resolution is the projection of this beamwidth on to the ground, which is given as follows

$$
\rho_{a}=\theta_{b w} R\left(t_{a}\right)=0.866 \frac{\lambda R\left(t_{a}\right)}{L_{a}}
$$

The real aperture resolution is typically on the order of several kilometers for a satellite SAR system [7].

\footnotetext{
${ }^{1}$ The terms "path length" and "phase delay" are sometimes used interchangeably in antenna literature

${ }^{2}$ Zero squint has been assumed here. The development can be easily extended to a non-zero squint case.
} 


\section{B.2 Synthetic Aperture}

Figure B.2 shows the sensor track as it moves along in azimuth direction over a target. The synthetic aperture length $L_{s}$ is the distance in azimuth at which the target remains in the $3-\mathrm{dB}$ (real-aperture) beamwidth of the antenna. From the geometry of Figure B.2. this is the arc-length $\mathbf{A C}$, which is given as follows

$$
L_{s}=\theta_{\text {syn }} R_{0}
$$

where $\theta_{\text {syn }}$ is known as the "synthetic angle", and $R_{0}$ is the point target range when the target is in the center of the beam. Different approximations exist in literature for $\theta_{\text {syn }}$ depending on the earth model and the scene geometry under consideration. For a flat earth geometry, $\theta_{\text {syn }}=\theta_{b w}$, which is the definition commonly used for the airborne case [7]. For the spaceborne case, an approximation for $\theta_{\text {syn }}$ is given in [7]

$$
\theta_{s y n}=\theta_{b w} \frac{V_{\mathrm{a}}}{V_{\mathrm{g}}}
$$




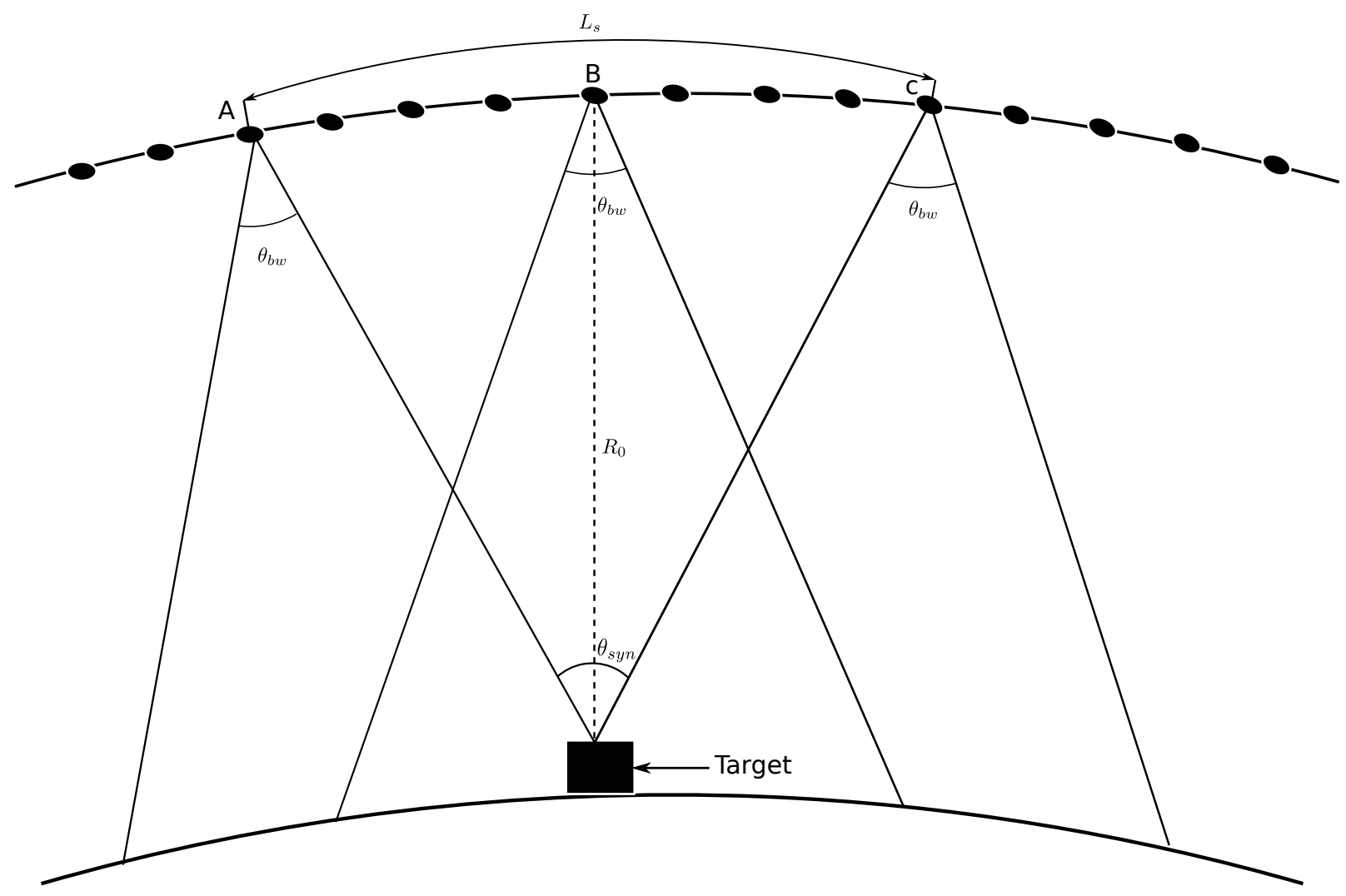

Figure B.2: Azimuth beamwidth and antenna locations where the pulses are transmitted and received, illustrating the concept of synthetic aperture. 


\section{Appendix $\mathrm{C}$}

\section{Stationary Phase Approximation}

This chapter provides the derivation of the stationary phase approximation that has been used to evaluate the signal derivative in Equation 2.40a. The assumption behind stationary phase approximation is that the signal derivative does not change when the phase is changing rapidly, and only changes when the phase is near stationary. This approximation has been used to compute the derivatives for SAR signals in [7].

The idea behind stationary phase approximation is that when the phase of sinusoidal functions oscillates rapidly as the frequency changes, the sinusoidal functions will interfere constructively at some points and destructively at other points, leading to an incoherent summation. This causes the Fourier integral to decay rapidly, except around the point where the phase is stationary. Note that in order for the stationary phase approximation to work, the amplitude should be slowly-varying compared to the phase. This assumption holds for the type of linear FM (chirp) signals examined in this thesis.

The general problem is to find the derivative of function with rapidly oscillating phase. This problem arises when evaluating the fourier transform as follows

$$
I=\int_{-\infty}^{\infty} F(x) e^{-j \phi(x)} d x
$$

where the phase $\phi(x)$ is rapidly osciallating and $F(x)$ is smooth or slowly varying by comparison.

Taylor series expansion of the phase term $\phi(x)$ around $x=x_{s}$. Note that the phase 
is stationary when $\phi^{\prime}\left(x_{s}\right)=0$.

$$
\begin{gathered}
I \approx \int_{-\infty}^{\infty} F\left(x_{s}\right) e^{-j \phi\left(x_{s}\right)+\frac{1}{2} \phi^{\prime \prime}\left(x_{s}\right)\left(x-x_{s}\right)^{2}} d x \\
I \approx F\left(x_{s}\right) e^{-j \phi\left(x_{s}\right)} \int_{-\infty}^{\infty} e^{-j \frac{1}{2} \phi^{\prime \prime}\left(x_{s}\right)\left(x-x_{s}\right)^{2}} d x
\end{gathered}
$$

Now apply a change of variable and use polar co-ordinates to solve the integral:

$$
\begin{array}{r}
H=\int e^{-j \alpha u^{2}} d u \\
H^{2}=\int e^{-j \alpha u^{2}} d u \int e^{-j \alpha v^{2}} d v \\
H^{2}=\left(\iint e^{-j \alpha\left(u^{2}+v^{2}\right)} d u d v\right)
\end{array}
$$

Convert to polar co-ordinates:

$$
\begin{array}{r}
r^{2}=u^{2}+v^{2} \\
u=r \cos \theta \\
v=r \sin \theta \\
d u d v=r d r d \theta
\end{array}
$$

Substituting the relations above in Equation C.3c;

$$
\begin{array}{r}
H^{2}=\int_{0}^{2 \pi} \int_{0}^{-\infty} e^{-j \alpha r^{2}} r d r d \theta \\
H^{2}=\int_{0}^{2 \pi} \int_{0}^{-\infty} \frac{-1}{2} e^{j \alpha u} d u d \theta \\
H^{2}=\int_{0}^{2 \pi}\left[\frac{-1}{2 j \alpha} e^{j \alpha u}\right]_{0}^{-\infty} \\
H^{2}=\int_{0}^{2 \pi} \frac{1}{2 j \alpha} d \theta=\frac{\pi}{j \alpha} \\
H=\sqrt{\frac{\pi}{j \alpha}}
\end{array}
$$

Substituting Equation C.5e into Equation C.2b:

$$
\begin{array}{r}
I=\left.F\left(x_{s}\right) e^{-j \phi\left(x_{s}\right)} \sqrt{\frac{\pi}{j \alpha}}\right|_{\alpha=\phi^{\prime \prime}\left(x_{s}\right)} \\
I=F\left(x_{s}\right) e^{-j \phi\left(x_{s}\right)} \sqrt{\frac{2 \pi}{j \phi^{\prime \prime}\left(x_{s}\right)}}
\end{array}
$$




\section{Appendix D}

\section{MODEX Modes}

The SMTI performance of any system depends on the signal level compared to the clutter plus noise signal levels [47]. To that end, different measurement modes have been developed that measure the scene from different spatial and temporal positions, using different antenna patterns and system settings. For RADARSAT-2, a set of measurement modes have been developed for GMTI, which are collectively known as Moving Object Detection Experiment (MODEX) [19]. A subset of these modes are shown in Figure D.1 and are discussed in this section.

The illustration on the top-left of Figure D.1 shows the radar configuration for the standard MODEX-1 mode. In this measurement mode, the full antenna aperture is used for transmitting and half aperture is used for receiving, utilizing the two physical phasecentres/channels of RADARSAT-2. This configuration allows the radar to make an acquisition using two receive channels. The two MODEX-2 modes are illustrated in the top-right and bottom for Figure D.1. In these modes, the spatial diversity of the standard MODEX-1 mode is increased by transmitter toggling, which provides additional receive channels (virtual channels) with the potential to improve GMTI performance. However, the increase in spatial diversity comes at a cost of increasing the PRF of the radar, which is typically doubled when using any of the MODEX-2 modes. Higher PRF usually requires more expensive phase-shifters and other hardware that might not be feasible for a space-borne system.

In MODEX-2 1/2 toggle transmit mode (top-right), the first and last half of the transmit aperture are alternatively switched on. As shown in Figure D.1, this mode provides three independant phase-centres of receive channels, which are shown as downpointing triangles. Same strategy is used for MODEX-2 3/4, however, $3 / 4$ of the transmit 

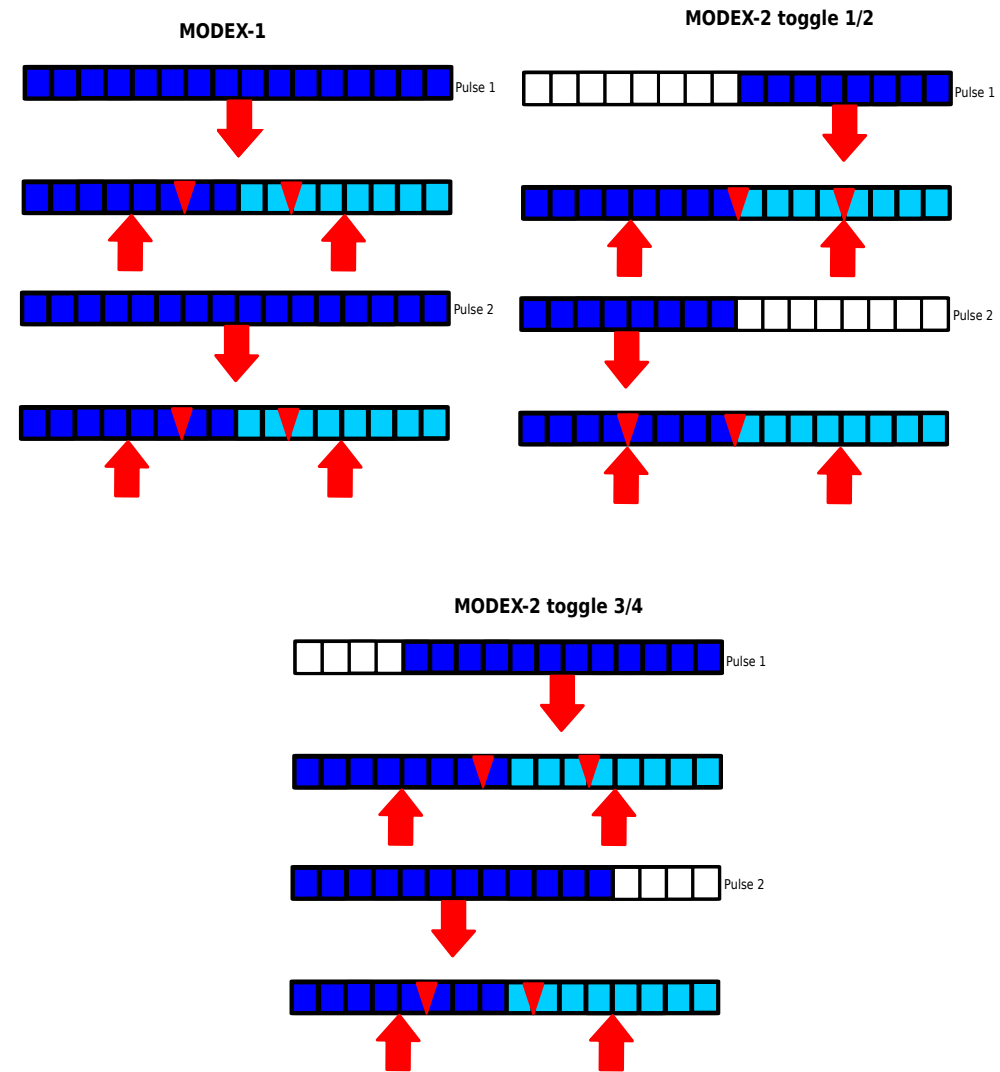

Figure D.1: RADARSAT 2 MODEX modes: (top-left) standard two-channel receive mode, (top-right) three-channel half aperture toggle-transmit mode, (bottom) fourchannel quarter aperture toggle-receive mode. Shaded rectangles represent active antenna panels with different shades representing different channels; down/up arrows represent transmitter/receiver physical center positions respectively; down-pointing triangles represent two-way effective phase centers.

aperture is used. However, this mode provides four independant phase-centres or receive channels. The MODEX-2 1/2 mode has the advantage of maintaining the same phasecentre distance (or the along-track baseline), but it comes at the cost of a decrease in the transmit power and the attainable SNR, which could severely impact the GMTI performance [19]. In comparison, the MODEX-2 3/4 mode doesn't suffer from such a high loss of SNR. 


\section{D.1 Space-Time Sampling}

The temporal and spatial sampling of the MODEX modes of Figure D.1 is presented in Figure D.2. For optimal GMTI performance, the samples must be taken at the same spatial position for the different RX channels but at different time. This is known as the DPCA condition [19]. Among other specifications, the different GMTI modes are designed to meet the optimal sampling criteria (DPCA condition), however, due to the limitations of space-borne SAR systems, it is difficult, if not impossible, to achieve optimal sampling. For one, achieving the DPCA condition and high spatial diversity (virtual channels) requires very high PRF, which is typically beyond the capabilities of the system. Therefore, trade-offs are made when designing different switching/toggling schemes (modes).

Figure D.1 shows that for MODEX-2 3/4, with a PRF of $3750 \mathrm{~Hz}$, all four phasecentres are almost aligned in space at different times. Whereas for MODEX-1 and MODEX-2 1/2, only two phase-centres are almost aligned in space at different times. Therefore, according to the DPCA condition criterion, MODEX-2 3/4 mode would be better suited for GMTI as it provides the highest spatial diversity and it comes closest to meeting the optimal sampling condition. 

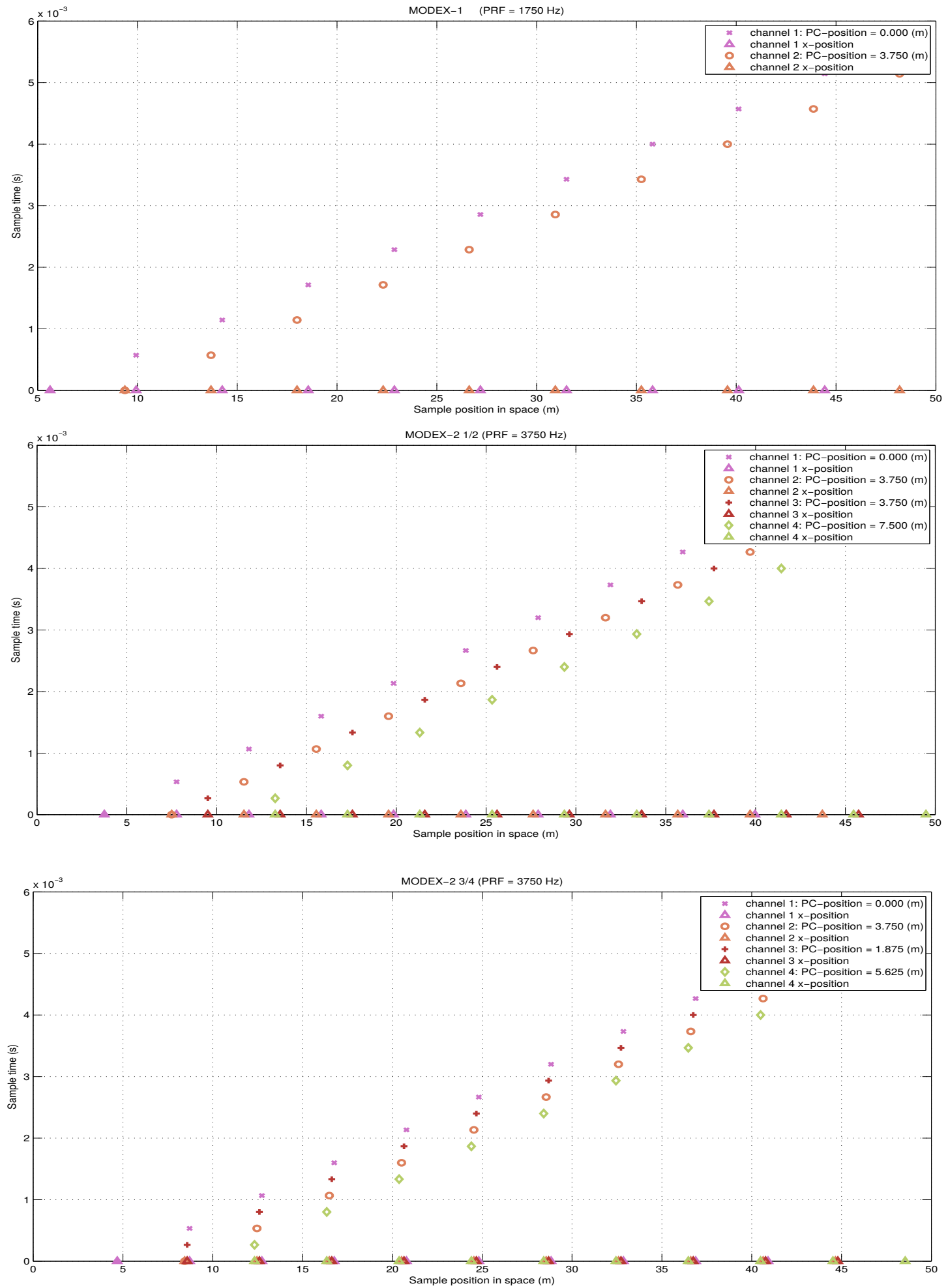

Figure D.2 


\section{Appendix E}

\section{SNR and CNR}

Two important parameters that significantly affect the radar performance are signal-tonoise ratio (SNR) and clutter-to-noise ratio (CNR). The expressions for these parameters are derived in this section.

\section{E.1 Signal-To-Noise Ratio}

For a discrete target, the SNR is the ratio of the received target signal power $P_{r}$ and the thermal noise power $P_{n}$.

$$
S N R=\frac{P_{r}}{P_{n}}
$$

\section{Received Target Power}

A radar transmits a pulse towards a target at range $R_{0}$ with transmit power $P_{t x}$. The power density of a directional antenna is given as follows [16]:

$$
Q_{i}=\frac{P_{t x} G_{t x}}{4 \pi R_{0}^{2}}
$$

where $G_{t x}$ is the transmit antenna gain.

This transmitted pulse is reflected by the target in many different directions. The reflected power is a function of the target radar cross section (RCS) $\sigma_{s}$ given in $m^{2}$.

$$
P_{r e f l}=Q_{i} \sigma_{s}=\frac{P_{t x} G_{t x} \sigma_{s}}{4 \pi R_{0}^{2}} .
$$


Similar to the expression for transmitted power density towards a target at range $R_{0}$, the received power density is derived by dividing the reflected power $P_{r e f l}$ by the surface-area of a sphere with radius $R_{0}$ (i.e the distance between the radar and the target).

$$
Q_{r}=\frac{P_{r e f l}}{4 \pi R_{0}^{2}} .
$$

The received power from a target at $R_{0}$ is given as the received power density times the effective antenna area.

$$
P_{r}=Q_{r} A_{e}
$$

where $A_{e}$ is the effective antenna area. An expression of the effective antenna area is often given in terms of the antenna gain as follows:

$$
A_{e}=\frac{\lambda^{2} G}{4 \pi},
$$

where $\lambda$ is the signal wavelength and $G$ is the antenna gain.

Using equation E.4, E.5, and E.6, the expression for received signal power from a target at range $R_{0}$ is written as follows:

$$
P_{r}=\frac{P_{t x} G_{t x} G_{r x} \lambda^{2} \sigma_{s}}{(4 \pi)^{3} R_{0}^{4}},
$$

where $G_{r x}$ is the gain of the receive antenna. Equation E.8 denotes the received target power under ideal conditions. In practice, power losses due to various factors must be taken into account. A detailed discussion of power losses is beyond the scope of this report but is provided in [16]. Letting $L_{s}$ denote the total system power loss, the expression for the total target power can be rewritten as follows:

$$
P_{r}=\frac{P_{t x} G_{t x} G_{r x} \lambda^{2} \sigma_{s}}{(4 \pi)^{3} R_{0}^{4} L_{s}}
$$

\section{Receiver Thermal Noise}

The receiver thermal noise, or "white" noise, is uniformly distributed over all radar frequencies (i.e. constant power spectral density). For a particular radar, these range of frequencies are determined by the radar bandwidth $B$

$$
P_{n}=K_{B} T_{s} B=K_{B} T_{0} N_{f} B,
$$


where

- $K_{B}$ is the Boltzmann's constant

- $T_{s}$ is the system noise temperature

- $T_{0}$ is the standard temperature $(290 K)$

- $B$ is the radar bandwidth

- $N_{f}$ is the noise figure of the receiver subsystem

The noise figure is typically given in $d B$, but must be converted to linear units to be used in Equation E.9.

Using equations E.8 and E.9, the expression for SNR from a single transmit pulse is given as follows:

$$
S N R=\frac{P_{t x} G_{t x} G_{r x} \lambda^{2} \sigma_{s}}{(4 \pi)^{3} R_{0}^{4} K_{B} T_{0} N_{f} B L_{s}} .
$$

For GMTI applications, the signal SNR after range compression is what affects the performance of detection and estimation. SNR after range compression takes into account the gain from range compression, which is given as follows:

$$
G_{r c}=B T_{r}
$$

where $B$ is signal bandwidth and $T_{r}$ is the pulse duration. The final expression for SNR after range compression is given by

$$
S N R_{r c}=S N R \cdot G_{r c}=\frac{P_{t x} G_{t x} G_{r x} \lambda^{2} T_{r} \sigma_{s}}{(4 \pi)^{3} R_{0}^{4} K_{B} T_{0} N_{f} L_{s}}
$$

\section{E.1.1 Integrated SNR}

A SAR system performs coherent integration of received pulses. As a result, there is signal gain provided by the total number of pulses received over the $4 \mathrm{~dB}$ width of the effective one-way antenna given by

$$
N_{\text {int }}=\frac{R_{0} \lambda f_{p r f}}{L_{a} V_{\mathrm{a}}},
$$


where $L_{a}$ is the effective one-way antenna length, and $V_{\mathrm{a}}$ is platform velocity. The integrated SNR is then given as follows

$$
\begin{array}{r}
S N R_{i n t}=N_{i n t} S N R, \\
S N R_{\text {int }}=\frac{P_{t x} G_{t x} G_{r x} \lambda^{3} f_{p r f} T_{r} \sigma_{s}}{(4 \pi)^{3} R_{0}^{3} K_{B} T_{0} N_{f} L_{a} V_{\mathrm{a}} L_{s}} .
\end{array}
$$

\section{E.2 Clutter-To-Noise Ratio}

The CNR is derived in a similar manner to SNR in Equation E.12, but with the target RCS $\sigma_{s}$ replaced by the total RCS of a clutter range-azimuth resolution cell, which is given as follows:

$$
\begin{array}{r}
\sigma_{c}=\sigma_{0} \rho_{r} \rho_{a} \\
\sigma_{c}=\sigma_{0} \frac{c}{2 K_{r} T_{r}} \frac{0.866 \lambda R_{0}}{L_{a}}
\end{array}
$$

where the range-azimuth resolution is given as the product of the range resolution $\rho_{r}$ of Equation 2.4 and the real azimuth resolution of Equation B.4, and $\sigma_{0}$ is the clutter reflectivity. The CNR from a single pulse is then given as follows:

$$
C N R_{r c}=\frac{P_{t x} G_{t x} G_{r x} \lambda^{2} T_{r}}{(4 \pi)^{3} R_{0}^{4} K_{B} T_{0} N_{f} L_{s}} \frac{c}{2 K_{r} T_{r}} \frac{\lambda R_{0}}{L_{a}} \sigma_{0} 0.866 .
$$

\section{Integrated CNR}

The integrated CNR is computed in a similar manner to the integrated SNR as shown in Equation E.14b, but target RCS is replaced by the total synthetic RCS of a clutter range-azimuth resolution cell, which is given as follows:

$$
\begin{array}{r}
\sigma_{c}=\sigma_{0} \rho_{r} \rho \\
\sigma_{c}=\sigma_{0} \frac{c}{2 K_{r} T_{r}} \frac{L_{a}}{2} \frac{V_{\mathrm{g}}}{V_{\mathrm{a}}}
\end{array}
$$

Note that unlike Equation E.15b, the synthetic azimuth resolution of Equation 2.62 has been used in the above expression. The integrated CNR is written as follows: 


$$
C N R_{\text {int }}=\frac{P_{t x} G_{t x} G_{r x} \lambda^{3} f_{p r f} T_{r}}{(4 \pi)^{3} R_{0}^{3} K_{B} T_{0} N_{f} L_{a} V_{\mathrm{a}} L_{s}} \sigma_{0} \frac{c}{2 K_{r} T_{r}} \frac{L_{a}}{2} \frac{V_{\mathrm{g}}}{V_{\mathrm{a}}}
$$

